\title{
Generalizing Bayesian phylogenetics to infer shared evolutionary events
}

\author{
Jamie R. Oaks ${ }^{* 1}$, Perry L. Wood, Jr. ${ }^{1}$, Cameron D. Siler², and Rafe M. \\ Brown $^{3}$ \\ ${ }^{1}$ Department of Biological Sciences \& Museum of Natural History, Auburn \\ University, Auburn, Alabama 36849 \\ ${ }^{2}$ Sam Noble Oklahoma Museum of Natural History and Department of \\ Biology, University of Oklahoma, Norman, Oklahoma 73072-7029 \\ ${ }^{3}$ Biodiversity Institute and Department of Ecology and Evolutionary Biology, \\ University of Kansas, Lawrence, Kansas 66045, USA
}

October 17, 2021

\begin{abstract}
Many processes of biological diversification can simultaneously affect multiple evolutionary lineages. Examples include multiple members of a gene family diverging when a region of a chromosome is duplicated, multiple viral strains diverging at a "superspreading" event, and a geological event fragmenting whole communities of species. It is difficult to test for patterns of shared divergences predicted by such processes, because all phylogenetic methods assume that lineages diverge independently. We introduce a Bayesian phylogenetic approach to relax the assumption of independent, bifurcating divergences by expanding the space of topologies to include trees with shared and multifurcating divergences. This allows us to jointly infer phylogenetic relationships, divergence times, and patterns of divergences predicted by processes of diversification that affect multiple evolutionary lineages simultaneously or lead to more than two descendant lineages. Using simulations, we find the new method accurately infers shared and multifurcating divergence events when they occur, and performs as well as current phylogenetic methods when divergences are independent and bifurcating. We apply our new approach to genomic data from two genera of geckos from across the Philippines to test if past changes to the islands' landscape caused bursts of speciation. Unlike our previous analyses restricted to only pairs of gecko populations, we find evidence for patterns of shared divergences. By generalizing the space of phylogenetic trees in a way that is independent from the likelihood model, our approach opens many avenues for future research into processes of diversification across the life sciences.
\end{abstract}

KEY WORDS: Phylogenetics, Bayesian, shared divergence, multifurcation.

${ }^{*}$ Corresponding author: joaks@auburn.edu 


\section{Introduction}

There are many processes of biological diversification that affect multiple evolutionary lineages, generating patterns of temporally clustered divergences across the tree of life. Understanding such processes of diversification has important implications across many fields and scales of biology. At the scale of genome evolution, the duplication of a chromosome segment harboring multiple members of a gene family causes multiple, simultaneous (or "shared") divergences across the phylogenetic history of the gene family (Doyle and Egan, 2010; Jiao et al., 2011; Clark and Donoghue, 2017; Li et al., 2018). In epidemiology, when a pathogen is spread by multiple infected individuals at a social gathering, this will create shared divergences across the pathogen's "transmission tree" (Pybus and Rambaut, 2009; Ypma et al., 2013; Klinkenberg et al., 2017). If one of these individuals infects two or more others, this will create a multifurcation (a lineage diverging into three or more descendants) in the transmission tree. At regional or global scales, when biogeographic processes fragment communities, this can cause shared divergences across multiple affected species (Hickerson et al., 2006; Leaché et al., 2007; Plouviez et al., 2009; Voje et al., 2009; Daza et al., 2010; Barber and Klicka, 2010). If the landscape is fragmented into three or more regions, this can also cause multifurcations (Hoelzer and Meinick, 1994). We are limited in our ability to infer patterns of divergences predicted by such processes, because phylogenetic methods assume lineages diverge independently.

To formalize this assumption of independent divergences and develop ways to relax it, it is instructive to view phylogenetic inference as an exercise of statistical model selection where each topology is a separate model (Yang, 1994; Yang et al., 1995; Suchard et al., 2001). Current methods for estimating rooted phylogenies with $N$ tips only consider tree models with $N-1$ bifurcating divergences, and assume these divergences are independent, conditional on the topology (see Lewis et al., 2005, for multifurcations in unrooted trees). If, in the history leading to the tips we are studying, diversification processes affected multiple lineages simultaneously or caused them to diverge into more than two descendants, the true tree could have shared or multifurcating divergences. This would make current phylogenetic models with $N-1$ independent divergence times over-parameterized, introducing unnecessary error (Figure 1). Even worse, with current methods, we lack an obvious way of using our data to test for patterns of shared or multifurcating divergences predicted by such processes.

We relax the assumption of independent, bifurcating divergences by introducing a Bayesian approach to generalizing the space of tree models to allow for shared and multifurcating divergences. In our approach, we view trees with $N-1$ bifurcating divergences as only one class of tree models in a greater space of trees with anywhere from 1 to $N-1$ potentially shared and/or multifurcating divergences. We introduce reversible-jump Markov chain Monte Carlo algorithms (Metropolis et al., 1953; Hastings, 1970; Green, 1995) to sample this generalized space of trees, allowing us to jointly infer evolutionary relationships, shared and multifurcating divergences, and divergence times. We couple these algorithms with a likelihood model for directly calculating the probability of biallelic characters given a population (or species) phylogeny, while analytically integrating over all possible gene trees under a coalescent model and all possible mutational histories under a finite-sites model of character evolution (Bryant et al., 2012; Oaks, 2019). Using simulations, we find the generalized tree model accurately infers shared and multifurcating divergences while maintaining a low rate of falsely inferring 


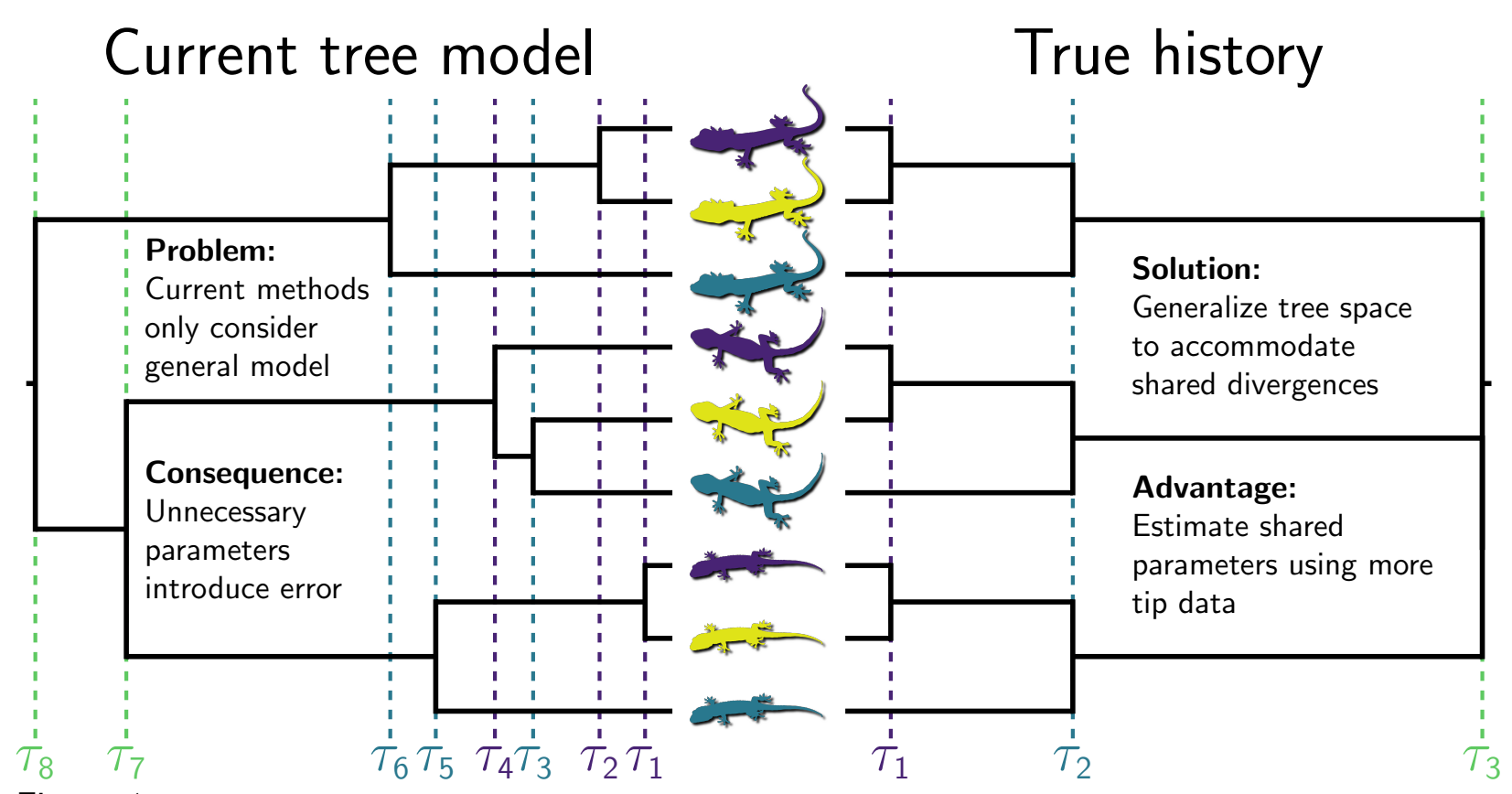

Figure 1. An example evolutionary history with shared divergences, and the benefits of the generalizing tree space under such conditions (left). Current methods are restricted to one class of tree models, where the tree is fully bifurcating and independent divergence-time parameters are estimated for all internal nodes (right). Figure made using Gram (Version 4.0.0; Foster, 2018) and the P4 phylogenetic toolkit (Version 1.4 5742542; Foster, 2004). Middle three lizard silhouettes from pixabay.com, and others from phylopic.org; all licensed under the Creative Commons (CCO) Public Domain Dedication.

such divergences. To test for patterns of shared and multifurcating divergences predicted by repeated fragmentation of the Philippines by interglacial rises in sea level (Oaks et al., 2013; Brown et al., 2013; Oaks et al., 2019), we apply the generalized tree model to genomic data from two genera of geckos codistributed across the islands.

\section{Results}

\subsection{Simulations on fixed trees}

The generalized tree model $\left(M_{G}\right)$ sampled trees significantly closer (Robinson and Foulds, 1979; Kuhner and Felsenstein, 1994) to the true tree than an otherwise equivalent model that assumes independent, bifurcating divergences $\left(M_{I B}\right)$, when applied to 100 data sets simulated along the species tree in Figure 2A, each with 50,000 unlinked biallelic characters (Figure 2B). From these simulated data, the generalized model consistently inferred the correct shared and multifurcating divergences with high posterior probabilities (Figure 2C). Unlike the independent-bifurcating model, the generalized approach avoids strong support for nonexistent branches that spuriously split truly multifurcating nodes (Figure 2D). Under both models, analyzing only the variable characters causes a reduction in tree accuracy (Figure 2B), but yields similar posterior probabilities for shared and multifurcating divergences (Figure 2C). 


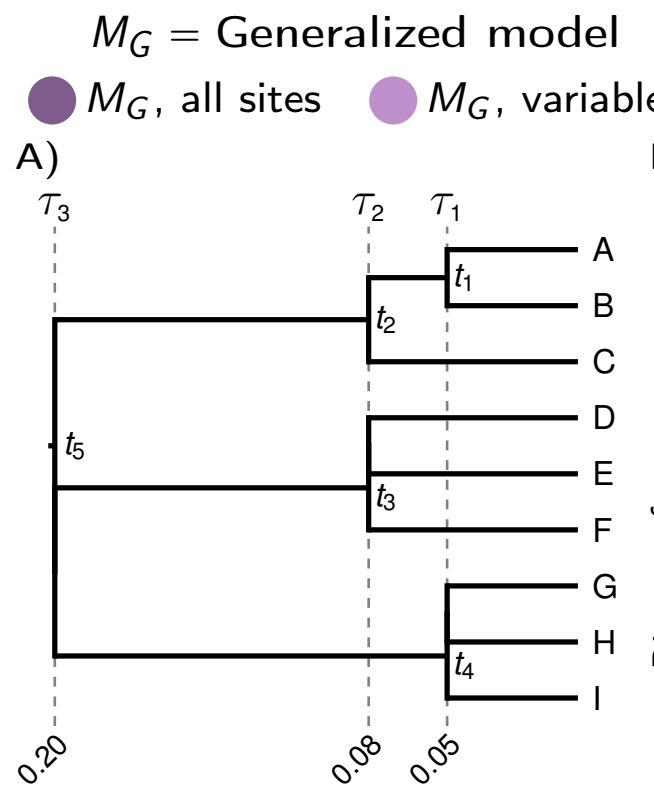

\section{$M_{I B}=$ Independent-bifurcating model}

B)
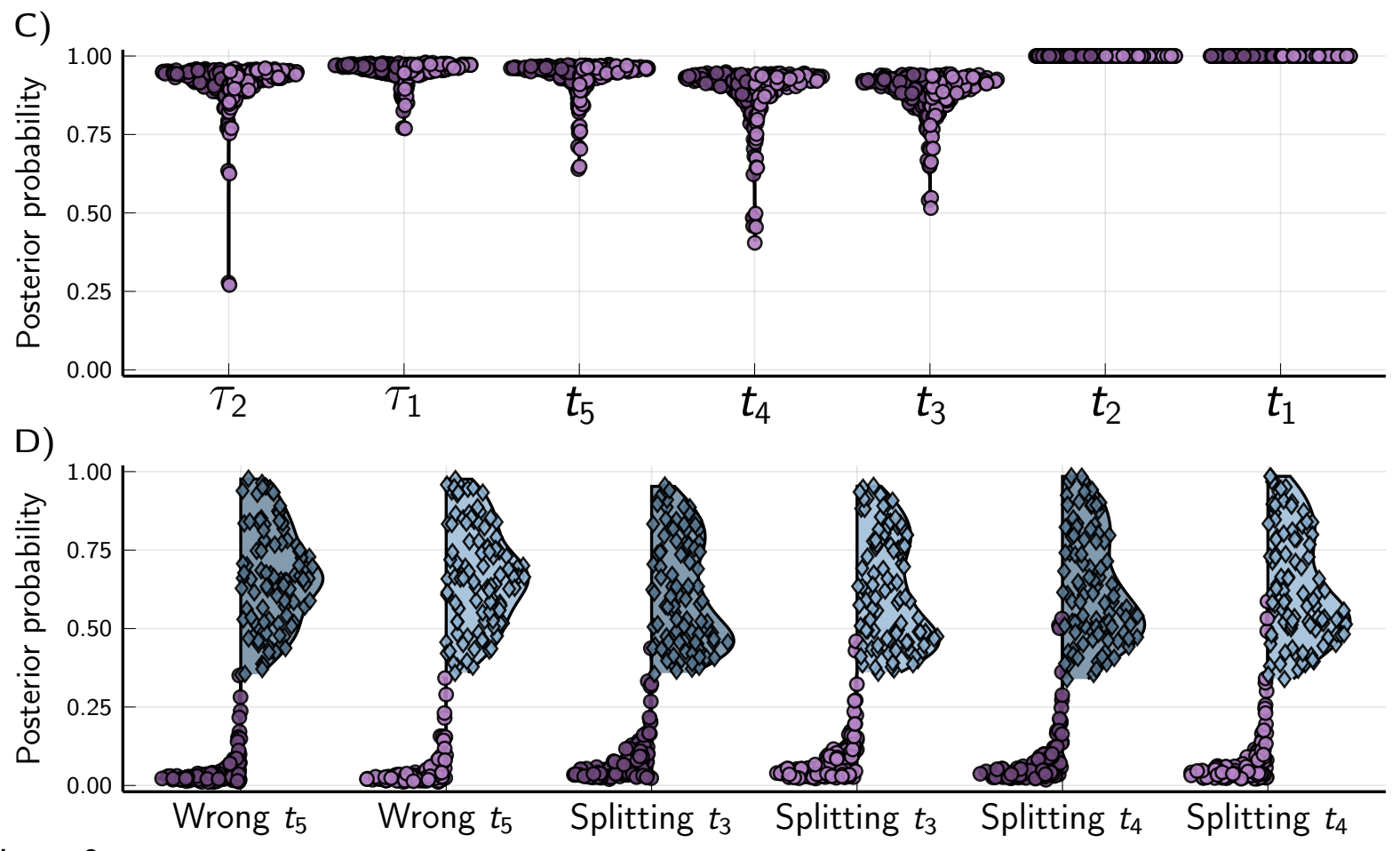

Figure 2. Results of analyses of 100 data sets, each with 50,000 biallelic characters simulated on the species tree shown in (A) with divergence times in units of expected substitutions per site. (B) The square root of the sum of squared differences in branch lengths between the true tree and each posterior tree sample (Kuhner and Felsenstein, 1994); the point and bars represent the posterior mean and equal-tailed $95 \%$ credible interval, respectively. Pvalues are shown for Wilcoxon signed-rank tests (Wilcoxon, 1945) comparing the paired differences in tree distances between methods. (C) Violin plots of the posterior probabilities of each node and shared divergence in the true tree across the 100 simulated data sets. (D) Violin plots of the most probable incorrect root node and most probable of the three incorrect splittings of the $t_{3}$ and $t_{4}$ multifurcations. For each simulation, the mutation-scaled effective population size $\left(N_{e} \mu\right)$ was drawn from a gamma distribution (shape $=20$, mean $=0.001$ ) and shared across all the branches of the tree; this distribution was used as the prior in analyses. Tree plotted using Gram (Version 4.0.0, Commit 02286362; Foster, 2018) and the P4 phylogenetic toolkit (Version 1.4, Commit d9c8d1b1; Foster, 2004). Other plots created using the PGFPlotsX (Version 1.2.10, Commit 1adde3d0; Carlsson and Papp, 2021) backend of the Plots (Version 1.5.7, Commit f80ce6a2; Breloff, 2021) package in Julia (Version 1.5.4; Bezanson et al., 2017). 
When applied to data sets simulated along a tree with independent, bifurcating divergences (Figure 3A), both the $M_{G}$ and $M_{I B}$ models consistently inferred the correct topology with strong support (Figure $2 \mathrm{~B}$ ), and the $M_{G}$ method did not support incorrect shared or multifurcating divergences (Figure $3 \mathrm{C}$ ). This was true whether all the characters or only the variable characters were analyzed (Figure 3B\&C). Looking at the distances (Robinson and Foulds, 1979; Kuhner and Felsenstein, 1994) between the trees from the posterior samples and the true tree, there is no difference between the $M_{G}$ and $M_{I B}$ models when the true tree has only independent, bifurcating divergences (Figure 3D). For both models, using all the characters yields posterior samples of more accurate trees than only analyzing variable characters (Figure 3D).

\subsection{Simulations on random trees}

When we simulated 100 data sets where the true tree and divergence times were randomly drawn from the generalized tree distribution $\left(M_{G}\right)$, we again found that the $M_{G}$ performs better than the $M_{I B}$ at inferring the correct tree and divergence times (Figure 4A), and generally recovers true shared and multifurcating divergences with moderate to strong support (Figure $4 \mathrm{~B} \& \mathrm{C}$ ). When the tree and divergence times were randomly drawn from an independent, bifurcating tree model $\left(M_{I B}\right)$, the generalized model performs similarly to the true model (Figure S1).

Both the $M_{G}$ and $M_{I B}$ models accurately and precisely estimate the age of the root, tree length, and effective population size from the data sets simulated on random $M_{G}$ and $M_{I B}$ trees (Top two rows of Figures S2, S3, and S4, respectively). Accuracy is similar with and without constant characters, but precision is higher when including constant characters.

\subsection{The rate of falsely inferring shared divergences}

To quantify the rate at which phycoeval incorrectly infers shared and/or multifurcating divergences, we used the results from the $M_{G}$ analyses of the data sets simulated on random trees from the $M_{I B}$ model. From the posterior sample of each analysis, we used sumphycoeval to calculate the proportion of samples that contained incorrectly merged neighboring divergence times. To do this, we merged all seven possible neighboring divergence times from the true tree, each of which creates a shared divergence or multifurcation, and counted how many samples contained each divergence scenario. We found that phycoeval had a low false-positive rate for the simulated data; less than $5 \%$ of incorrectly merged divergence times had an approximate posterior probability greater than 0.5 (Figure 5 and Figure S5). In all cases with moderate to strong support for falsely merged divergences, the difference in time between the merged divergences was small $(<0.005$ expected substitutions per site; Figure 5). There was no pattern between support for incorrectly merged divergences and their age (Figure 5).

\subsection{Convergence and mixing of MCMC chains}

For all analyses of simulated data, the root age, tree length, and effective population size had a potential-scale reduction factor (PSRF; the square root of Equation 1.1 in Brooks and 


\section{$M_{G}=$ Generalized model $\quad M_{I B}=$ Independent-bifurcating model}

$M_{G}$, all sites $\bigcirc M_{G}$, variable sites $\neg M_{I B}$, all sites $>M_{I B}$, variable sites

A)

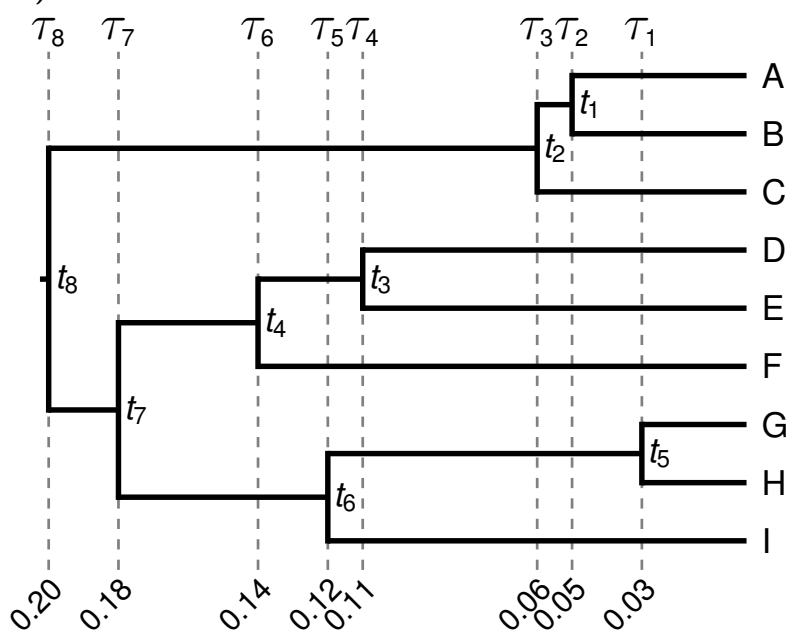

B)

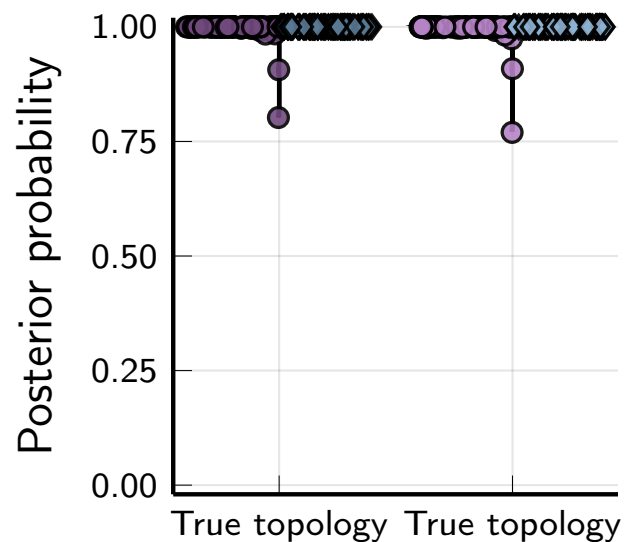

C)

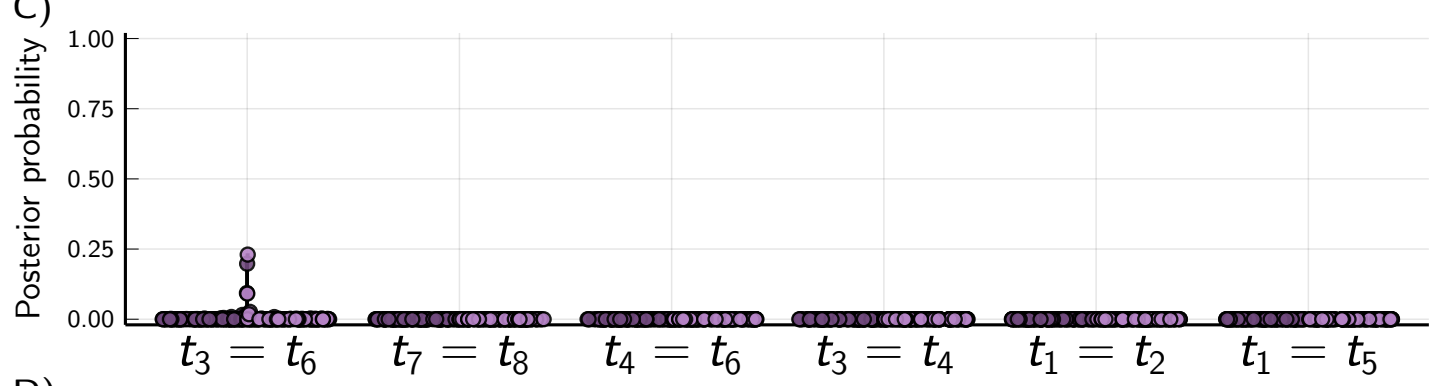

D)

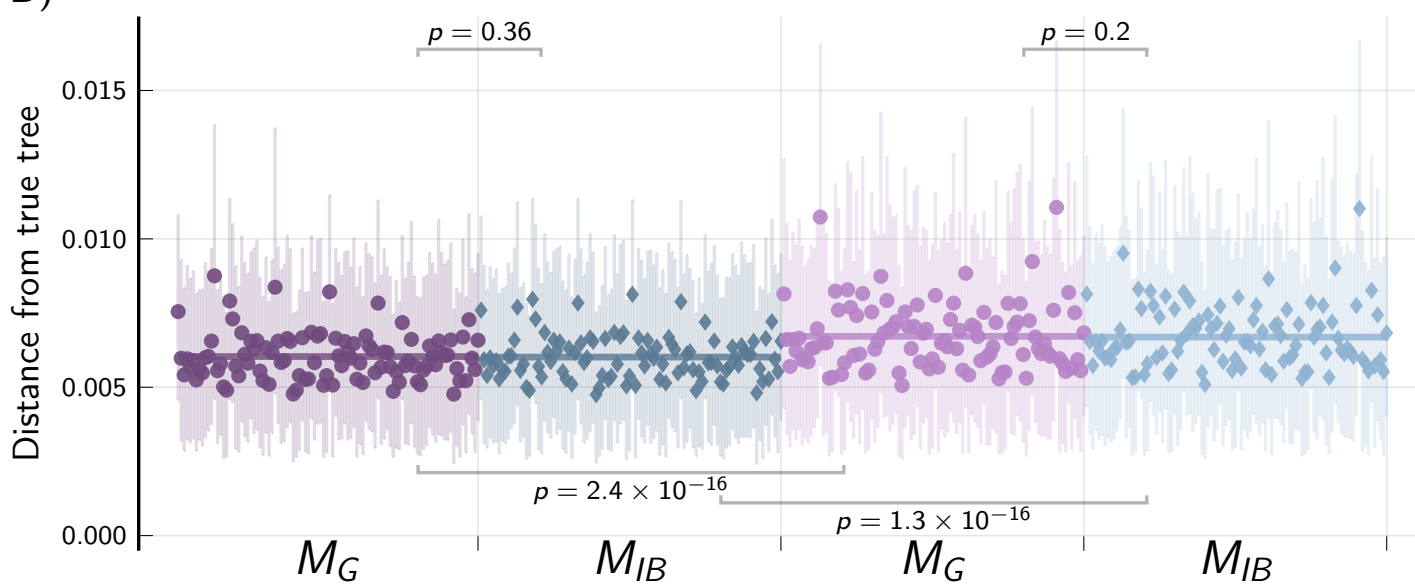

Figure 3. Results of analyses of 100 data sets, each with 50,000 biallelic characters simulated on the species tree shown in (A) with divergence times in units of expected substitutions per site. (B) The posterior probability of the true topology. (C) The posterior probability of incorrectly shared or multifurcating nodes. (D) The square root of the sum of squared differences in branch lengths between the true tree and each posterior tree sample (Kuhner and Felsenstein, 1994); the point and bars represent the posterior mean and equal-tailed 95\% credible interval, respectively. P-values are shown for Wilcoxon signed-rank tests (Wilcoxon, 1945) comparing the paired differences in tree distances between methods. For each simulation, the mutation-scaled effective population size $\left(N_{e} \mu\right)$ was drawn from a gamma distribution (shape $=20$, mean $\left.=0.001\right)$ and shared across all the branches of the tree; this distribution was used as the prior in analyses. Tree plotted using Gram (Version 4.0.0, Commit 02286362; Foster, 2018) and the P4 phylogenetic toolkit (Version 1.4, Commit d9c8d1b1; Foster, 2004). Other plots created using the PGFPlotsX (Version 1.2.10, Commit 1adde3d0; Carlsson and Papp, 2021) backend of the Plots (Version 1.5.7, Commit f80ce6a2; Breloff, 2021) package in Julia (Version 1.5.4; Bezanson et al., 2017). 
bioRxiv preprint doi: https://doi.org/10.1101/2021.07.23.453597; this version posted October 17, 2021. The copyright holder for this preprint (which was not certified by peer review) is the author/funder, who has granted bioRxiv a license to display the preprint in perpetuity. It is made available under aCC-BY 4.0 International license.
$M_{G}=$ Generalized model (true model)
$M_{I B}=$ Independent-bifurcating model
$M_{G}$, all sites $M_{G}$, variable sites
$M_{I B}$, all sites
$M_{I B}$, variable sites

A)

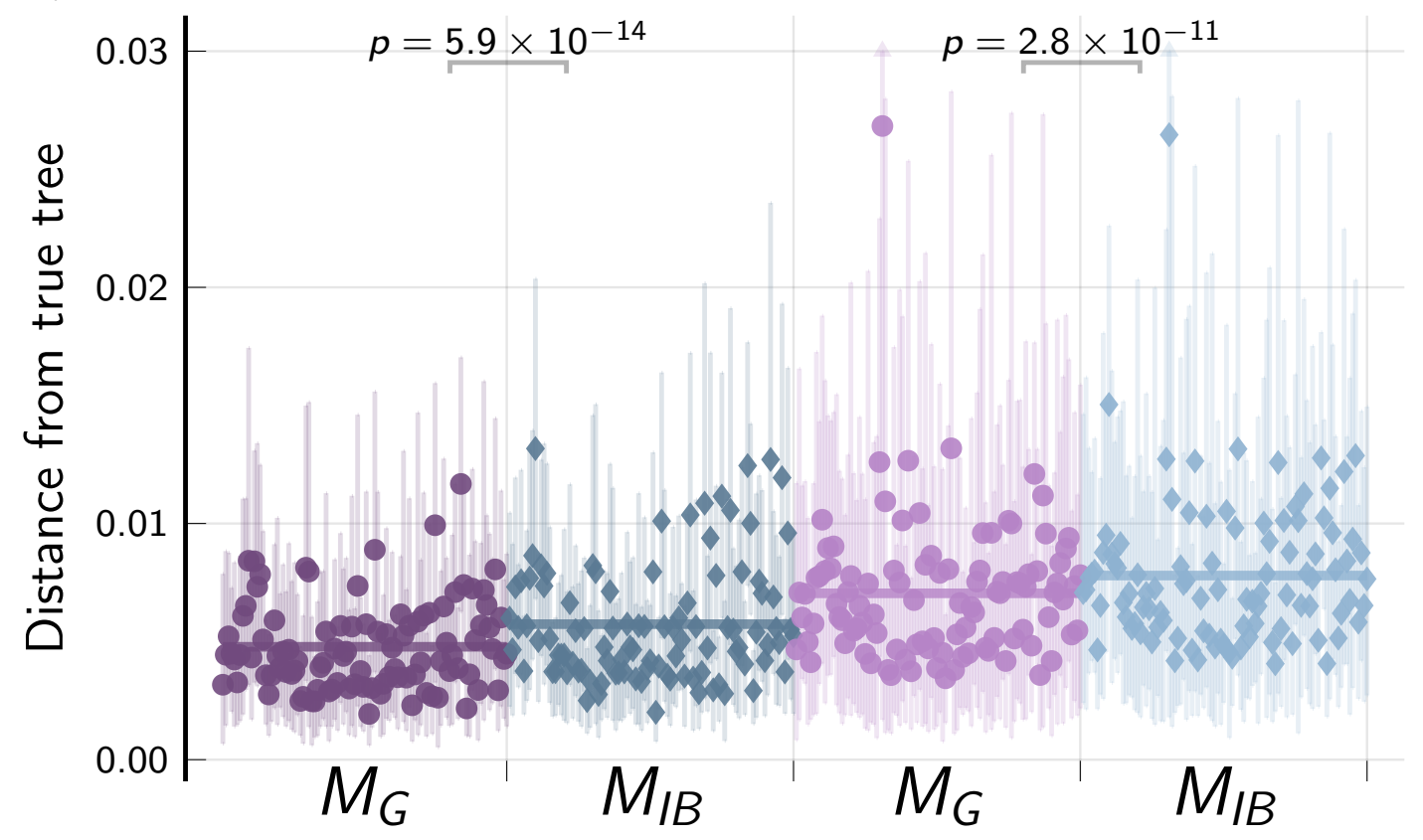

B)

C)
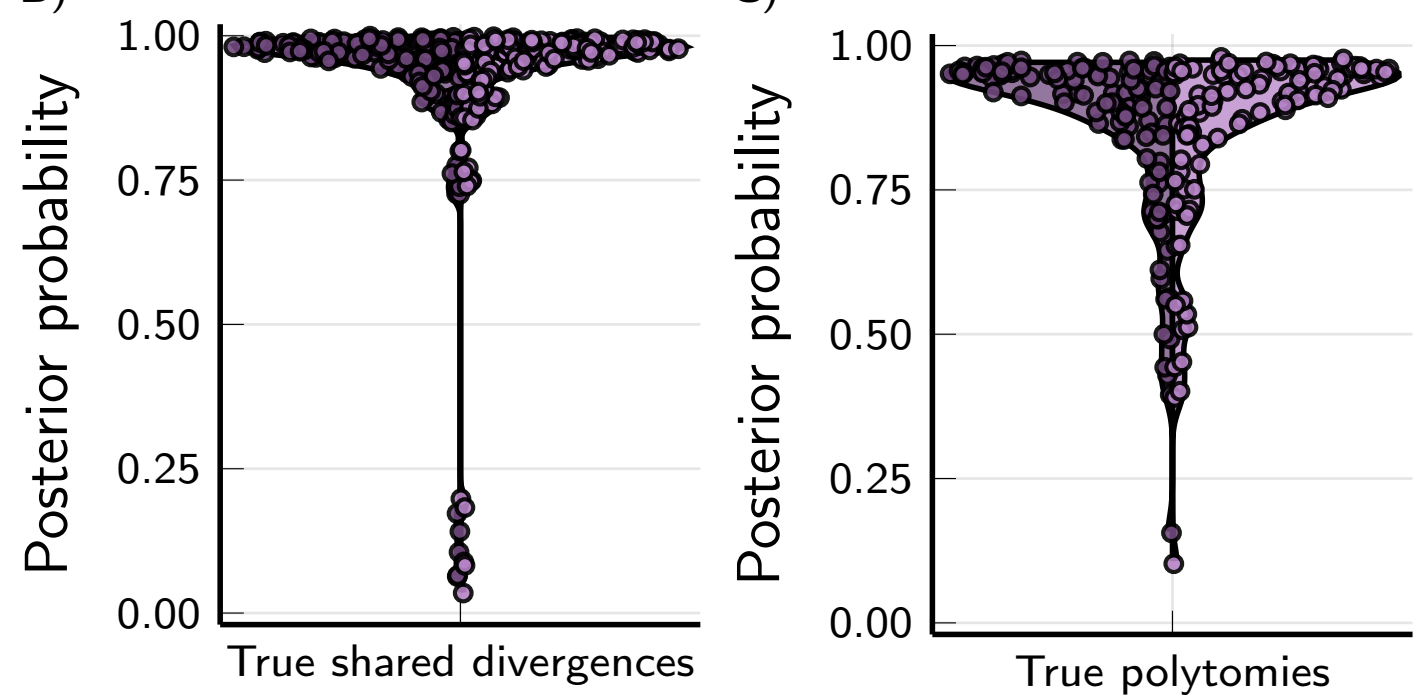

Figure 4. The performance of the $M_{G}$ and $M_{I B}$ tree models when applied to 100 data sets, each with 50,000 biallelic characters simulated on species trees randomly drawn from the $M_{G}$ tree distribution. (A) The square root of the sum of squared differences in branch lengths between the true tree and each posterior tree sample (Kuhner and Felsenstein, 1994); the point and bars represent the posterior mean and equal-tailed 95\% credible interval, respectively. P-values are shown for Wilcoxon signed-rank tests (Wilcoxon, 1945) comparing the paired differences in tree distances between methods. Violin plots show posterior probabilities of all true (B) shared divergences and (C) multifurcating nodes across all simulated trees. For each simulation, the mutation-scaled effective population size $\left(N_{e} \mu\right)$ was drawn from a gamma distribution (shape $=20$, mean $=0.001$ ) and shared across all the branches of the tree; this distribution was used as the prior in analyses. Plots created using the PGFPlotsX (Version 1.2.10, Commit 1adde3d0; Carlsson and Papp, 2021) backend of the Plots (Version 1.5.7, Commit f80ce6a2; Breloff, 2021) package in Julia (Version 1.5.4; Bezanson et al., 2017). 


\section{Probability of incorrectly merged div times (true model $=M_{\mid \mathrm{B}}$ )}
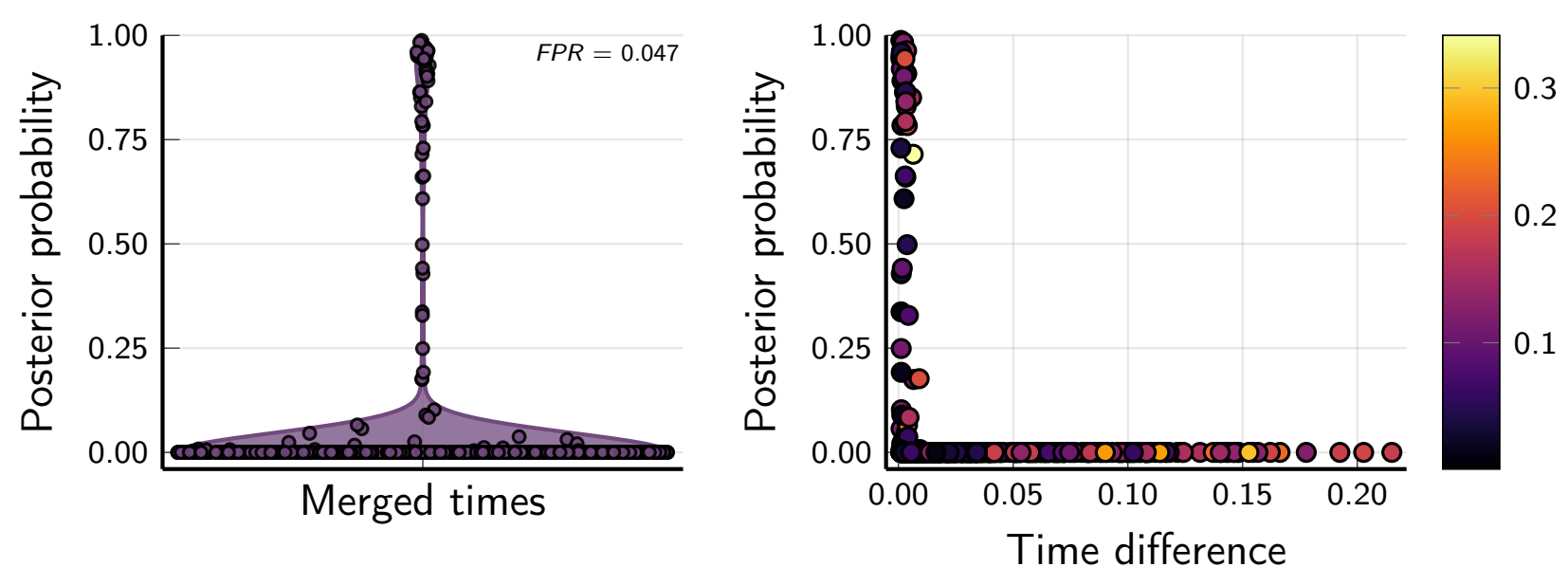

Figure 5. The $M_{G}$ has a low false positive rate (FPR; the proportion of incorrectly merged divergence times with a posterior probability $>0.5$ ) when applied to data simulated on trees drawn from $M_{I B}$ (no shared or multifurcating divergences). Support for incorrectly merged divergence times is high only when the difference between the times is small (right, X-axis, units of expected substitutions per site), and is not correlated with the age of the merged nodes (right, color gradient in units of expected substitutions per site). Plots created using the PGFPlotsX (Version 1.2.10, Commit 1adde3d0; Carlsson and Papp, 2021) backend of the Plots (Version 1.5.7, Commit f80ce6a2; Breloff, 2021) package in Julia (Version 1.5.4; Bezanson et al., 2017).

Gelman, 1998) less than 1.2 and effective sample size (ESS; Gong and Flegal, 2016) greater than 200. The average standard deviation of split frequencies (ASDSF) among the four MCMC chains was less than 0.017 for all analyses and less than 0.01 for most (Figure S6).

Convergence and mixing was better under $M_{G}$ than $M_{I B}$ when applied to data sets simulated on trees with shared or multifurcating divergences (left column of Figure S6). When applied to data sets simulated with no shared or multifurcating divergences, MCMC performance was similar between $M_{G}$ and $M_{I B}$ (right column of Figure S6).

\subsection{Simulations of linked characters}

The multi-species coalescent likelihood we have coupled with our generalized tree model assumes each biallelic character is unlinked (i.e., each character evolved along a gene tree that was independent of other characters, conditional on the species tree; Bryant et al., 2012; Oaks, 2019). We found the model is robust to violations of this assumption when applied to data simulated with linked characters. When we repeated the simulations above, but with 500 loci, each with 100 linked characters, our results are very similar when all the characters (variable and constant) are analyzed (Figures $\mathrm{S} 2-5$ and $\mathrm{S} 7-10$ ). When all but one variable character per locus is discarded to avoid violating the assumption of unlinked characters, performance is greatly reduced due to the large loss of data (Figures S2-5 and S7-10). These results suggest it is better to analyze all sites from multi-locus data sets, rather than reduce them to only one SNP per locus. 


\subsection{Testing for shared divergences in Philippine gekkonids predicted by glacial cycles}

The repeated fragmentation of the Philippines by interglacial rises in sea level since the late Pliocene (Haq et al., 1987; Rohling et al., 1998; Siddall et al., 2003; Miller et al., 2005; Spratt and Lisiecki, 2016) has been an important model to help explain remarkably high levels of microendemism and biodiversity across the archipelago (Inger, 1954; Heaney, 1985; Brown and Guttman, 2002; Evans et al., 2003; Heaney et al., 2005; Roberts, 2006; Linkem et al., 2010; Siler et al., 2010, 2011, 2012; Brown and Siler, 2014). This model predicts that recently diverged taxa across the islands should have (potentially multifurcating) divergence times clustered around the beginning of interglacial periods. We tested this prediction by applying our generalized tree model to RADseq data from species of Cyrtodactylus and Gekko collected from 27 and 26 locations across the islands, respectively (Tables S1 \& S2). We analyzed each genus separately, because the rate of mutation differs between the genera, and phycoeval currently assumes a strict clock.

The maximum a posteriori (MAP) trees for both genera had 16 divergence times and weak to moderate support for five shared divergences (Figure 6). The MAP tree of Cyrtodactylus and Gekko had three and two multifurcations, respectively. For both genera, two of the shared divergences involved three nodes, and of the remaining three that involved two nodes, one involved a trichotomous node (three descending lineages). There were no other strongly supported shared divergences that were not included in the MAP trees of either genera. Most of the shared and multifurcating divergences occurred after the late Pliocene, based on re-scaling the branch lengths of the posterior sample of trees from expected substitutions per site to millions of years using secondary calibrations (Figure 6).

For both genera, the number of divergence times with the highest approximate posterior probability (0.33 for Cyrtodactylus and 0.32 for Gekko) was 17, and the $95 \%$ credible interval spanned 15-19 divergences (Figure 6). No trees with more than 22 divergence times were sampled for either genera, making the approximate posterior probability of 23 or more divergences less than $2.9 \times 10^{-5}$ for both genera. The average standard deviation of split frequencies (0.0027 for Cyrtodactylus and 0.0009 for Gekko) and other statistics were consistent with the MCMC chains converging and mixing well (Table S4).

\section{Discussion}

To relax the assumption that all processes of biological diversification affect evolutionary lineages independently, we introduced a generalized Bayesian phylogenetic approach to inferring phylogenies with shared and multifurcating divergences. Using simulations we found this approach can accurately infer shared and multifurcating divergences from moderately sized data sets, while maintaining a low rate of incorrectly inferring such patterns of divergence. When we used the generalized approach to infer the evolutionary histories of two genera of gekkonid lizards across the Philippines, we found strong support against tree models assumed by current phylogenetic methods. The posterior probability of all trees with $N-1$ independent, bifurcating divergences was less than $2.9 \times 10^{-5}$ for both genera, suggesting that trees with shared and multifurcating divergences much better explain the gekkonid 
bioRxiv preprint doi: https://doi.org/10.1101/2021.07.23.453597; this version posted October 17, 2021. The copyright holder for this preprint (which was not certified by peer review) is the author/funder, who has granted bioRxiv a license to display the preprint in perpetuity. It is made available under aCC-BY 4.0 International license.

A
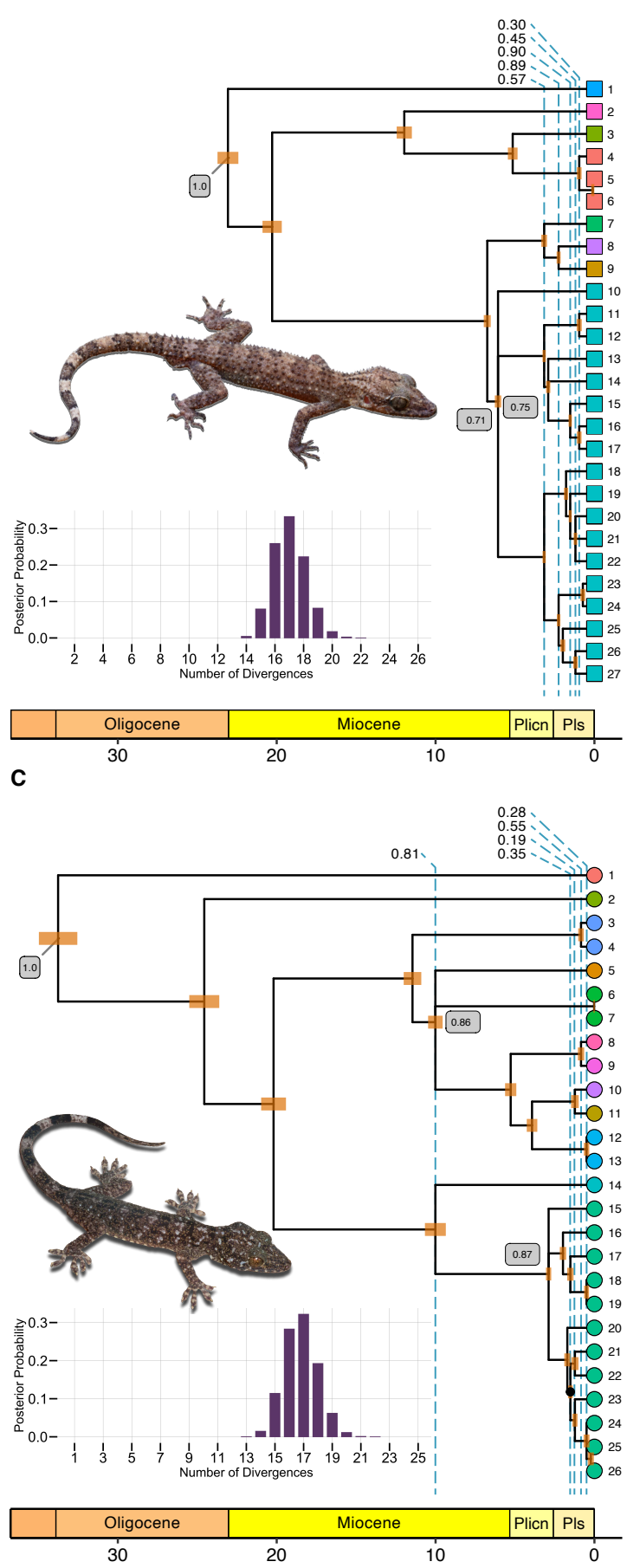

B
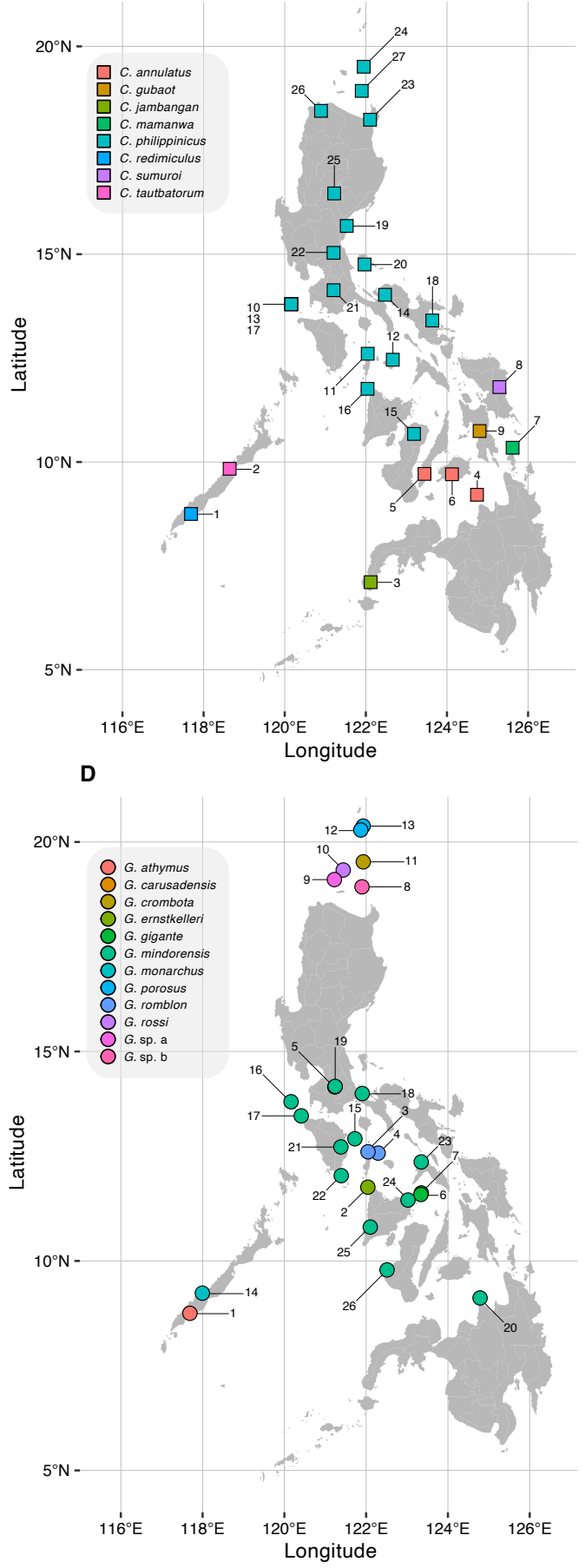

Figure 6. A summary of the generalized trees inferred from the Cyrtodactylus and Gekko RADseq data sets. The maximum a posteriori (MAP) tree is shown for both genera along with the approximate posterior probabilities of the number of divergences. Shared divergences in MAP trees indicated by dashed lines, with approximate posterior probabilities shown along the top. Dots at internal nodes inidicate splits with approximate posterior probabilities less than 0.95 (all nodes without dots are greater than 0.95). Approximate posterior probabilities of nodes shown in grey boxes for the root and multifurcating nodes. To illustrate timescale, branch lengths of posterior samples of trees were rescaled from expected substitutions per site to millions of years using secondary calibrations (see methods). Top photo of Cyrtodactylus sp. by CDS; bottom photo of Gekko sp. by Jason Fernandez \& RMB. Created using ggplot2 (v3.3.5; Wickham, 2016), ggtree (v3.1.0; Yu et al., 2017), treeio (v1.17.0; Wang et al., 2019), deeptime (v0.0.6; Gearty, 2021), cowplot (v1.1.1; Wilke, 2020), and ggrepel (v0.9.1; Slowikowski, 2020). Links to nexus-formatted annotated trees: Cyrtodactylus \& Gekko. 
sequence data.

Despite greatly expanding the number of possible topologies, we saw better MCMC behavior under the $M_{G}$ model (Figure S6). This could be due to the generalized tree distribution providing more ways to traverse tree space. For example, when a posterior distribution restricted to trees with independent bifurcating divergences has multiple "peaks" associated with different topologies, the generalized distribution includes tree models that are special cases of these topologies. Explicitly including these "intermediate" trees could make the posterior less rugged and allow MCMC chains to more easily traverse tree space.

By accommodating multifurcations, our generalized tree approach helped avoid the "startree paradox," where arbitrary resolutions of a true polytomy can be strongly supported (Figure 2D; Suzuki et al., 2002; Lewis et al., 2005). Lewis et al. (2005) found the same result by expanding the space of unrooted tree topologies to include multifurcations. Our results show that this solution to the star-tree paradox extends to rooted trees.

\subsection{Robustness of coalescent models that assume unlinked charac- ters}

Our finding that the multi-species coalescent model of Bryant et al. (2012) is robust to linked characters is consistent with previous simulations using species trees with one and two tips (Oaks, 2019; Oaks et al., 2019, 2020). Our simulation results show that this robustness extends to larger trees with multifurcations and shared divergences, and suggest that discarding data to avoid linked characters can have a worse effect on inference than violating the assumption of unlinked characters. This is consistent with the findings of Chifman and Kubatko (2014) that quartet inference of splits in multi-species coalescent trees from SNP data was also robust to the violation of the assumption that characters are unlinked.

\subsection{Diversification of Philippine gekkonid lizards}

How the 7,100 islands of the Philippines accumulated one of the highest concentrations of terrestrial biodiversity on Earth (Catibog-Sinha and Heaney, 2006; Brown and Diesmos, 2009; Heaney and Regalado, 1998; Brown et al., 2013) has been of interest to evolutionary biologists since the founding of biogeography (Wallace, 1869; Huxley, 1868; Dickerson, 1928; Diamond and Gilpin, 1983; Brown, 2016; Lomolino et al., 2016). Since the late Pliocene, the archipelago's five major (and several minor) aggregate island complexes were repeatedly fragmented by interglacial rises in sea level into clusters of landmasses resembling today's islands, followed by island fusion via land bridge exposure as sea levels fell during glacial periods (Haq et al., 1987; Rohling et al., 1998; Siddall et al., 2003; Miller et al., 2005; Spratt and Lisiecki, 2016). The repeated fragmentation-fusion cycles of this insular landscape has generated a prominent hypothesis to explain the high levels of terrestrial biodiversity across the Philippines (Inger, 1954; Heaney, 1985; Brown and Guttman, 2002; Evans et al., 2003; Heaney et al., 2005; Roberts, 2006; Linkem et al., 2010; Siler et al., 2010, 2011, 2012; Brown and Siler, 2014). However, there is growing evidence that (1) older tectonic processes ( 30-5 mya) of precursor paleoislands (Jansa et al., 2006; Blackburn et al., 2010; Siler et al., 2012; Brown and Siler, 2014; Brown et al., 2016), (2) dispersal events from mainland 
source populations (Diamond and Gilpin, 1983; Brown and Guttman, 2002; Brown and Siler, 2014; Chan and Brown, 2017), (3) repeated colonizations among islands (Siler et al., 2011; Justiniano et al., 2015; Brown et al., 2016), and (4) fine-scale in situ isolating mechanisms (Heaney et al., 2011; Linkem et al., 2011; Siler et al., 2011, 2012; Hosner et al., 2013; Brown et al., 2015), have been important causes of diversification among and within many of the islands.

Oaks et al. (2019) found support for independent divergence times among inter-island pairs of Cyrtodactylus and Gekko populations from across the Philippines, suggesting that dispersal might be a more important mechanism of isolation than sea-level fragmentation in these gekkonid lizards. Our fully phylogenetic approach to this problem has allowed us to look for shared divergences across the full evolutionary history of extant populations of these clades, finding evidence for shared divergences that were missed by the pairwise approach. These results emphasize a pitfall of previous methods: choosing pairs of populations, for comparison under previous methods for inferring shared divergences (Hickerson et al., 2006; Huang et al., 2011; Oaks, 2014, 2019), was problematic in the sense that it was somewhat arbitrary and could miss more complex patterns of shared divergences in the shared ancestry of the taxa under study.

Our findings of weak to moderate support for a small number of shared and multifurcating divergences during the diversification of Cyrtodactylus and Gekko is consistent with accumulating evidence that many different processes of diversification have played important roles across the Philippines, not just island fragmentation. Nonetheless, it remains possible, and in some ways likely, that a simultaneous analysis involving broader taxonomic sampling of Philippine gekkonids (e.g., Gekko, Cyrtodactylus, Pseudogekko, Lepidodactylus, and Luperosaurus; Wood, Jr. et al., 2020) would reveal greater support for an increased number of shared divergences across the archipelago. For example, early divergences in both genera show patterns consistent with early arrival into the archipelago, and subsequent diversification, via the Palawan Island Arc (Blackburn et al., 2010; Siler et al., 2012). Within Cyrtodactylus, divergence of $C$. redimiculus is inferred to have occurred between 20.41-19.28 mya, which appears consistent with diversification of the older, island and microendemic lineages of Gekko in the Philippines (e.g., G. romblon, G. carusadensis; Figure 6) that likely entered the West Visayan faunal region in the central Philippines via the same Palawan microcontinental block between 20.97-19.41 mya (Siler et al., 2012). Among the divergences inferred to have occurred more recently within the last $1.5 \mathrm{my}$, there also appear to be regional consistencies in when and where lineages were diversifying in the Philippines, including population-level diversification for the widespread Cyrtodactylus philippinicus and Gekko mindorensis within and among the Mindoro and West Visayan faunal regions in the central Philippines (Figure 6; Siler et al., 2012, 2014). Regardless of temporal concordance among divergences, the results of this work further support Philippine species within both focal clades having originated in the archipelago as a result of one or more faunal exchanges between oceanic portions of the Philippines associated historically with the Philippine mobile belt and the Palawan microcontinental block.

Currently, broader taxonomic analyses are limited by a simplifying assumption of phycoeval that mutation rates are constant across the tree. We sought to minimize the effects of violations of this assumption by analyzing the two gekkonid genera separately. The Philippine species in each genus are closely related (the posterior mean root age in expected substi- 
tutions per site for Cyrtodactylus and Gekko was 0.012 and 0.013, respectively) and share similar natural histories, so an assumption of a similar rate of mutation across the populations we sampled within each genus seems reasonable. Future developments of phycoeval allowing the rate to vary across the phylogeny would be an obvious way to improve our current implementation and make it more generally applicable to a greater diversity of systems.

\subsection{Future directions}

Given that processes of co-diversification are of interest to fields as diverse as biogeography, epidemiology, and genome evolution, we hope the generalized tree model offers a statistical framework for studying these processes across the life sciences. To help achieve this, there are several ways to improve upon our current implementation of this approach. Allowing the generalized tree model and associated MCMC algorithms to be coupled with a diverse set of phylogenetic likelihood models is an obvious way to expand its applicability to more data types and systems. The independence of the tree model and MCMC algorithms from the likelihood function makes this relatively straightforward. Similarly, our approach can be extended to accommodate tips sampled through time (Stadler, 2010; Heath et al., 2014; Gavryushkina et al., 2016; Stadler et al., 2018) and "relaxed-clock" models (Drummond et al., 2006; Drummond and Suchard, 2010; Heath et al., 2011). The former would allow for fossil and epidemiological data, and the latter would allow it to be applied to diverse sets of taxa that are expected to vary in their rates of mutation.

As we alluded to above when discussing MCMC behavior, expanding the set of tree models to include all possible non-reticulating topologies with one to $N-1$ divergence times could have important implications for the joint posterior distribution of phylogenetic models. Theoretical work to characterize this joint space is needed.

Lastly, the distribution we used over the generalized tree space (uniform over topologies with beta-distributed node heights) is motivated by mathematical convenience, rather than inspired by biological processes. Process-based models, like a generalized birth-death model, could provide additional insights. In addition to inferring phylogenies with shared or multifurcating divergences, process-based models would allow us to infer the macroevolutionary parameters that govern the rate of such divergences.

\section{Methods}

\subsection{Generalized tree model}

Let $T$ represent a rooted, potentially multifurcating tree topology with $N$ tips and $n(t)$ internal nodes $\boldsymbol{t}=t_{1}, t_{2}, \ldots t_{n(t)}$, where $n(t)$ can range from 1 (the "comb" tree) to $N-1$ (fully bifurcating, independent divergences). Each internal node $t$ is assigned to one divergence time $\tau$, which it may share with other internal nodes in the tree. We will use $\boldsymbol{\tau}=\tau_{1}, \ldots, \tau_{n(\tau)}$ to represent $n(\tau)$ divergence times, where $n(\tau)$ can also range from 1 to $N-1$, and every $\tau$ has at least one node assigned to it, and every node maps to a divergence time more recent than its parent (Figure S11).

To formalize a distribution across this space of generalized trees, we assume all possible 
topologies $(T)$ are equally probable. We also assume the age of the root node follows a parametric distribution (e.g., a gamma distribution), and each of the other divergence times is beta-distributed between the present $\left(\tau_{0}\right)$ and the height of the youngest parent of a node mapped to the divergence time (Figure S11). This was inspired by and related to the Dirichlet distribution on divergence times of Kishino et al. (2001), but we use beta distributions to make it easier to deal with the fact that under our generalized tree model, multiple nodes can be mapped to each divergence time. For additional flexibility, we allow a distribution to be placed on the alpha parameter of the beta distributions of all the non-root divergence times, which we denote as $\alpha_{\tau}$.

\subsection{Likelihood model}

To perform Bayesian phylogenetic inference under the generalized tree model, it can be coupled with any function for calculating the probability of data evolving along a tree. This means it can be coupled with any data type and associated phylogenetic likelihood function. Even if the likelihood function does not explicitly accommodate multifurcations, these can be treated as a series of arbitrary bifurcations with branches of zero length to obtain the same likelihood of the tree.

Here, we couple the generalized tree model with a multi-species coalescent model that allows the likelihood of any species tree to be estimated directly from biallelic character data, while analytically integrating out all possible gene trees and character substitution histories along those gene trees. Below we give a brief overview of this model; for a full description of this likelihood model, please see Bryant et al. (2012), and see Oaks (2019) for a correction when only variable characters are analyzed.

\subsubsection{The data}

From $N$ species for which we wish to infer a phylogeny, we assume we have collected orthologous, biallelic genetic characters. By "biallelic", we mean that each character has at most two states, which we refer to as "red" and "green" following Bryant et al. (2012). For each character from each species, we have collected $n$ copies of the locus, $r$ of which are copies of the red allele. We will use $\mathbf{n}$ and $\mathbf{r}$ to denote allele counts for one character from all $N$ species; i.e., $\mathbf{n}, \mathbf{r}=\left\{\left(n_{1}, r_{1}\right),\left(n_{2}, r_{2}\right), \ldots\left(n_{N}, r_{N}\right)\right\}$. We use $\mathbf{D}$ to represent these allele counts across all the characters.

\subsubsection{The evolution of characters}

We assume each character evolved along a gene tree $(g)$ according to a finite-characters, continuous-time Markov chain (CTMC) model, and the gene tree of each character is independent of the others, conditional on the species tree (i.e., the characters are effectively unlinked). We use $u$ and $v$ to denote the relative rate of mutating from the red to green state and vice versa, respectively, as a character evolves along a gene tree, forward in time (Bryant et al., 2012; Oaks, 2019). Thus, $\pi=u /(u+v)$. is the stationary frequency of the green state. We denote the overall rate of mutation as $\mu$, which we assume is constant across the tree (i.e., a "strict clock"). Because evolutionary change is the product of $\mu$ and 
time, when $\mu=1$, time is measured in units of expected substitutions per character. If a mutation rate per character per unit of time is given, then time is measured in those units (e.g., generations or years).

\subsubsection{The evolution of gene trees}

We assume the gene trees of each character branched according to a multi-species coalescent model within a single, shared, generalized species tree, where each branch $i$ represents a population with a constant effective size $N_{e}^{i}$ (Nielsen and Wakeley, 2001; Rannala and Yang, 2003; Liu and Pearl, 2007; Heled and Drummond, 2010; Bryant et al., 2012). We use $\boldsymbol{N}_{\boldsymbol{e}}$ to denote the effective population sizes for all branches in the generalized tree, with topology $T$ and divergence times $\boldsymbol{\tau} ; \boldsymbol{N}_{\boldsymbol{e}}=N_{e}^{1}, N_{e}^{2}, \ldots, N_{e}^{n(t)+N}$ where $n(t)+N$ is equal to the number of branches in the tree.

\subsubsection{The likelihood}

Using the work of Bryant et al. (2012), we analytically integrate over all possible gene trees and character substitution histories to compute the likelihood of the species tree directly from all $m$ biallelic characters under a multi-population coalescent model (Kingman, 1982a,b; Rannala and Yang, 2003),

$$
p\left(\mathbf{D} \mid T, \boldsymbol{\tau}, \boldsymbol{N}_{\boldsymbol{e}}, \mu, \pi\right)=\prod_{i=1}^{m} p\left(\mathbf{n}_{i}, \mathbf{r}_{i} \mid T, \boldsymbol{\tau}, \boldsymbol{N}_{\boldsymbol{e}}, \mu, \pi\right)
$$

To accommodate multifurcations, we used recursion and Equation 19 of Bryant et al. (2012). This equation shows how to obtain the conditional probabilities at the bottom of an ancestral branch by merging the conditional probabilities at the top of its two descendant branches. At a multifurcation, we recursively apply Equation 19 of Bryant et al. (2012) to merge the conditional probabilities of each descendant branch in arbitrary order. We confirmed that this recursion returns an identical likelihood as treating the multifurcation as a series of bifurcations with zero-length branches.

\subsection{Bayesian inference}

The joint posterior probability distribution of the tree (with potential shared and multifurcating divergences) and other model parameters is

$$
p\left(T, \boldsymbol{\tau}, \alpha_{\tau}, \boldsymbol{N}_{\boldsymbol{e}}, \mu, \pi \mid \mathbf{D}\right)=\frac{p\left(\mathbf{D} \mid T, \boldsymbol{\tau}, \boldsymbol{N}_{\boldsymbol{e}}, \mu, \pi\right) p(T) p\left(\boldsymbol{\tau} \mid T, \alpha_{\tau}\right) p\left(\boldsymbol{N}_{\boldsymbol{e}}\right) p(\mu) p(\pi) p\left(\alpha_{\tau}\right)}{p(\mathbf{D})}
$$

\subsubsection{Priors}

We use the generalized tree distribution described above as the prior on the topology $(T)$ and divergence times $(\boldsymbol{\tau})$. For all of our analyses below, we (1) set the alpha parameter of the beta distributions on non-root divergence times $\left(\alpha_{\tau}\right)$ to $1,(2)$ set the mutation rate $(\mu)$ to 1 , so that time is in units of expected substitutions per character, (3) assume one 
gamma-distributed effective population size is shared across all the branches of the species tree, and (4) set the stationiary frequencies of the two character states to be equal $(\pi=0.5)$, making our CTMC model of character evolution a two-state equivalent to the "JC69" model of nucleotide substitution (Jukes and Cantor, 1969).

\subsection{Approximating the posterior of generalized trees}

We use Markov chain Monte Carlo (MCMC) algorithms (Metropolis et al., 1953; Hastings, 1970; Green, 1995) to sample from the joint posterior in Equation 2. To sample across trees with different numbers of divergence times during the MCMC chain, we use reversiblejump MCMC (Green, 1995). We also use univariate and multivariate Metropolis-Hastings algorithms (Metropolis et al., 1953; Hastings, 1970) to update the divergence times and effective population sizes. See the Supporting Information for details and validations of our MCMC algorithms.

\subsection{Software implementation}

We implemented the models and algorithms above for approximating the joint posterior distribution of generalized trees, divergence times, and other model parameters in the software package ecoevolity Oaks (2019); Oaks et al. (2019, 2020). The C++ source code for ecoevolity is freely available from https://github.com/phyletica/ecoevolity and includes an extensive test suite. From the $\mathrm{C}++$ source code, three command-line tools are compiled for generalized tree analyses: (1) phycoeval, for performing Bayesian inference under the model described above, (2) simphycoeval for simulating data under the model described above, and (3) sumphycoeval for summarzing the posterior samples of generalized trees collected by phycoeval. Documentation for how to install and use the software is available at http://phyletica.org/ecoevolity/. A detailed, version-controlled history of this project, including all of the data and scripts needed to produce our results, is available as a GitHub repository https://github.com/phyletica/phycoeval-experiments and was archived on zenodo (Oaks, 2021). We used multiple commits of ecoevolity for the analyses below, as we added features to the sumphycoeval tool (this history is documented in the project repository). However, all of our analyses can be replicated using Version 1.0.0 (Commit 2ed8d6ec) of ecoevolity.

\subsection{Simulation-based analyses}

\subsubsection{Methods used for all our simulations (unless noted)}

We used sumphycoeval to simulate data sets of 50,000 biallelic characters from one diploid individual from nine species (i.e., two copies of each character sampled from each species). Except for our simulations of linked characters described below, the characters were unlinked (i.e., each character was simulated along an independent gene tree within the species tree). For all of our simulations and analyses, we constrained the branches of the species tree to share the same diploid effective population size $\left(N_{e}\right)$, which we randomly drew 
from a gamma distribution with a shape of 20 and mean of 0.001 . We used this distribution as the prior on $N_{e}$ in subsequent analyses of the simulated data sets.

We analyzed each simulated data set under two models using phycoeval: the generalized tree model described above, which we denote as $M_{G}$, and an otherwise equivalent model that is constrained to the space of trees with independent, bifurcating divergences (i.e., trees with $N-1$ divergence times), which we denote as $M_{I B}$. For both $M_{G}$ and $M_{I B}$, we used a gammadistributed prior on the age of the root node with a shape of 10 and mean of 0.2 . For each data set we ran four independent MCMC chains for 15,000 generations, sampling every 10 generations, and retaining the last 1000 samples of each chain to approximate the posterior (4000 total samples). For each generation, nine (equal to the number of tips) MCMC moves are randomly selected in proportion to specified weights, some of which automatically call other moves after finishing to improve mixing. Each chain started from a random bifurcating topology with no shared divergences, and the root age and other divergence times drawn randomly from their respective prior distributions.

From the 4000 posterior samples collected for each simulated dataset, we used sumphycoeval to calculate the mean and $95 \%$ credible intervals of the root age, tree length, effective population size, and the number of divergence times, and to summarize the frequency of sampled topologies, splits, nodes, and shared divergences. We define a split as a branch in the tree that "splits" the tips of the tree into two non-overlapping subsets; those that do and do not descend from the branch. We define a node as a split with a particular set of splits that descend from it; this is necessary to summarize the frequency of multifurcations. We also used sumphycoeval to calculate the distance between every sampled tree and the true tree using the square root of the sum of squared differences in branch lengths (Robinson and Foulds, 1979; Kuhner and Felsenstein, 1994). To assess convergence and mixing of the chains, we used sumphycoeval calculate the average standard deviation of split frequencies (ASDSF; Lakner et al., 2008) across the four chains with a minimum split frequency threshold of $10 \%$, as well as the potential scale reduction factor (PSRF; the square root of Equation 1.1 in Brooks and Gelman, 1998) and effective sample size (ESS; Gong and Flegal, 2016) of the log likelihood, root age, tree length, and effective population size.

\subsubsection{Simulations on fixed trees}

We used simphycoeval to simulate 100 data sets on two fixed trees with 9 species, one with shared and multifurcating divergences (Figure 2A) and the other with only bifurcating, independent divergences (Figure 3A). We analyzed each simulated data set under models $M_{G}$ and $M_{I B}$, both with and without constant characters; for the latter we specified for phycoeval to correct the likelihood for only sampling variable characters (Bryant et al., 2012; Oaks, 2019).

\subsubsection{Simulations on random trees}

Using simphycoeval, we also simulated 100 data sets on trees randomly drawn from the prior distributions of the $M_{G}$ and $M_{I B}$ models. As above, we analyzed each simulated data set with and without constant characters under the $M_{G}$ and $M_{I B}$ models. We used MCMC to sample trees randomly from the prior distributions of both models. More specifically, 
we used simphycoeval to (1) randomly assemble a strictly bifurcating tree with no shared divergences times, (2) run an MCMC chain of topology changing moves for a specified number of generations (we used 1000), and (3) draw the root age, other divergence times, and the effective population sizes randomly from their respective prior distributions. For each MCMC generation, nine (equal to the number of tips) topology changing moves were randomly selected in proportion to specified weights.

Due to the nested beta (uniform) distributions on non-root divergence times, some trees sampled from $M_{G}$ and $M_{I B}$ will have all or most of the divergence times close to zero. This happens when one of the oldest non-root divergences is randomly assigned a time near zero. For example, the trees shown in Figure $7 \mathrm{~A}-\mathrm{C}$ all have eight independent, bifurcating divergences. Given such trees, it is nearly impossible to differentiate independent divergences with a finite data set. It is also not clear what an investigator would want phycoeval to infer given a true tree like Figure $7 \mathrm{~A}$ with eight independent divergences. To avoid such extreme scenarios, we rejected any trees that had divergences times closer than 0.001 substitutions per site. This resulted in 61 and 201 trees being rejected in order to obtain 100 trees under the $M_{G}$ and $M_{I B}$ models, respectively. Despite this filtering, challenging tree shapes remained in our sample for simulations. For example, see the trees in Figure $7 \mathrm{D}-\mathrm{F}$, all with eight independent, bifurcating divergences.

\subsubsection{Simulations of linked characters}

The likelihood model above assumes characters are unlinked (i.e., they evolved along gene trees that are independent of one another conditional on the species tree). To assess the effect on inference of violating this assumption, we repeated the simulations and analyses above (for both fixed and random trees), but simulated 500 loci of 100 linked characters each (i.e. for each locus, 100 characters evolved along a shared gene tree). We used simphycoeval to simulate these data sets in two ways: (1) all 50,000 characters are simulated and retained, and (2) only (at most) one variable character is retained for each locus. For the latter data sets, characters are unlinked, but only (at most) 500 characters, all variable, are sampled. We analyzed all of these data sets under both the $M_{G}$ and $M_{I B}$ models. For data sets with only variable characters, we corrected the likelihood for not sampling constant characters (Bryant et al., 2012; Oaks, 2019).

\subsection{Inference of shared divergences in Philippine gekkonids}

We applied our new approach to two genera of geckos, Gekko and Cyrtodactylus, sampled across the Philippine Islands. We used the RADseq data of Oaks et al. (2019) available on the NCBI Sequence Read Archive (Bioproject PRJNA486413, SRA Study SRP158258).

\subsubsection{Assembling alignments}

We used ipyrad (Version 0.9.43; Eaton and Overcast, 2020) to assemble the RADseq reads into loci for both genera. All of the scripts and ipyrad parameter files we used to assemble the data are available in our gekkonid project repository (https://github.com/ phyletica/gekgo) archived on Zenodo (Oaks and Wood, Jr., 2021), and the ipyrad settings 


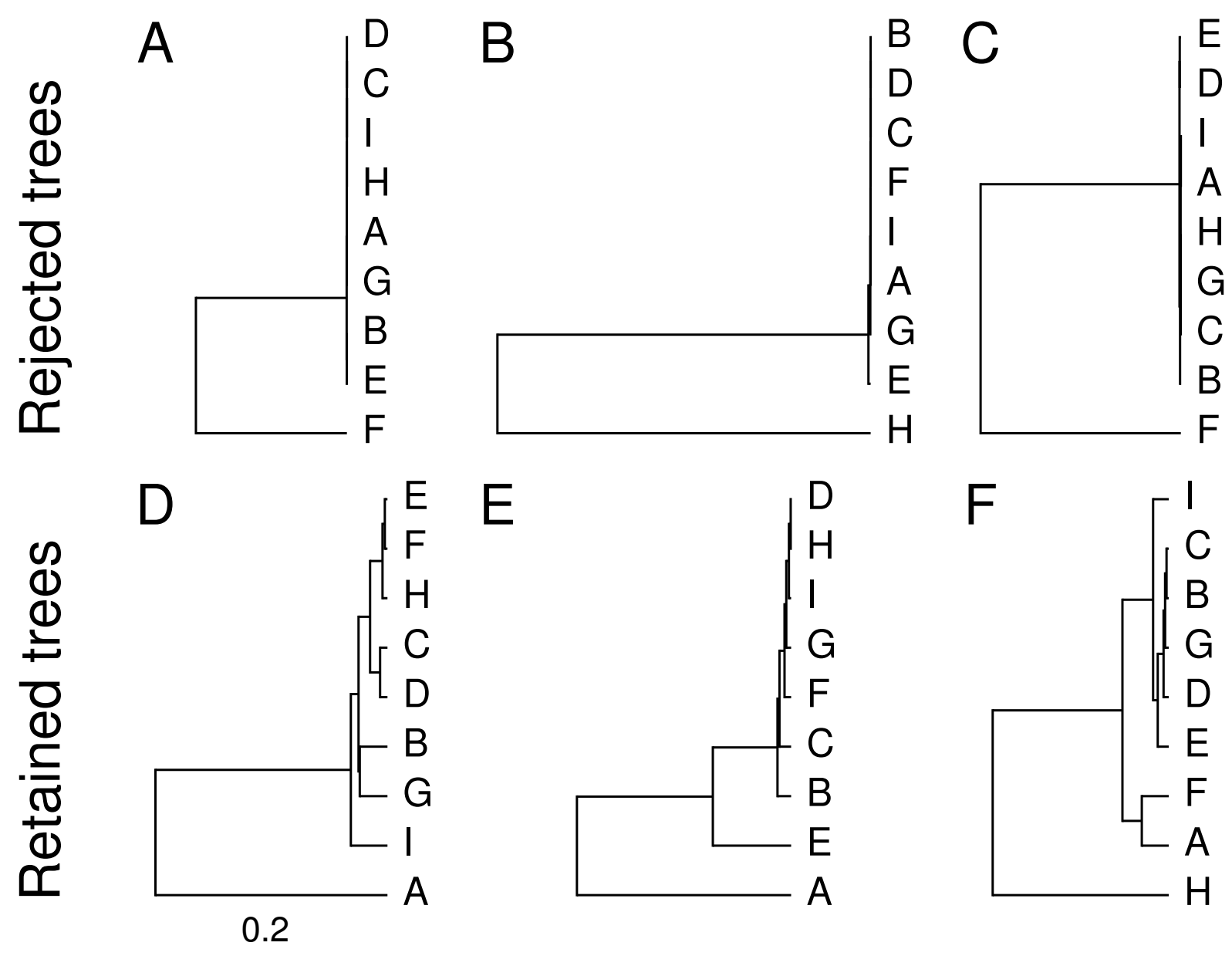

Figure 7. Examples of trees (all with 8 independent, bifurcating divergences) rejected (top) and retained (bottom) when a minimum threshold of 0.001 substitutions per site between divergence times is applied to trees randomly sampled from the prior distribution of the $M_{I B}$ model. Trees plotted using Gram (Version 4.0.0, Commit 02286362; Foster, 2018) and the P4 phylogenetic toolkit (Version 1.4, Commit d9c8d1b1; Foster, 2004). 
are listed in Table S3. Using pycoevolity (Version 0.2.9; Commit 217dbeea; Oaks, 2019), we converted the ipyrad alignments into nexus format, and in the process, removed sites that had more than two character states. The final alignment for Cyrtodactylus contained 1702 loci and 155,887 characters from 27 individuals, after 567 characters with more than two states were removed. The final alignment for Gekko contained 1033 loci and 94,612 characters from 26 individuals, after 201 characters with more than two states were removed. Both alignments had less than $1 \%$ missing characters. The assembled data matrices for Cyrtodactylus and Gekko are available in our project repository (https://github.com/ phyletica/phycoeval-experiments) and the data associated with specimens are provided in Tables S1 \& S2.

\subsubsection{Phylogenetic analyses}

When analyzing the Cyrtodactylus and Gekko character matrices with phycoeval, we (1) fixed stationary state frequencies to be equal $(\pi=0.5)$, (2) set the mutation rate $(\mu)$ to 1 so that divergence times are in units of expected substitutions per site, (3) used an exponentially distributed prior with a mean of 0.01 for the age of the root, (4) set $\alpha_{\tau}=1$ so that non-root divergence times are uniformly distributed between zero and the age of the youngest parent node, and (5) assumed a single diploid effective population size $\left(N_{e}\right)$ shared across the branches of the tree with a gamma-distributed prior. For the gamma prior on $N_{e}$, we used a shape of 2.0 and mean of 0.0005 for Cyrtodactylus, and a shape of 4.0 and mean of 0.0002 for Gekko, based on estimates of Oaks et al. (2019) from the same and related species.

For both genera, we ran 25 independent MCMC chains for 15,000 generations, sampling the state of the chain every 10 generations. In each generation, phycoeval attempts $N$ MCMC moves (27 and 26 for Cyrtodactylus and Gekko, respectively) randomly selected in proportion to specified weights, some of which automatically call other moves after finishing to improve mixing. For 20 of the chains, we specified for phycoeval to start from the "comb" topology $(n(\tau)=1)$. For the remaining five chains, we had phycoeval start with a random bifurcating topology with no shared divergences $(n(\tau)=N-1)$.

We used sumphycoeval to summarize the sampled values of all parameters and the frequency of sampled topologies, splits, nodes, and shared divergences. To assess convergence and mixing, we used sumphycoeval to calculate the average standard deviation of split frequencies (ASDSF; Lakner et al., 2008) and the potential scale reduction factor (PSRF; the square root of Equation 1.1 in Brooks and Gelman, 1998) and effective sample size (ESS; Gong and Flegal, 2016) of all parameters across all 25 MCMC chains. We present these convergence statistics in Table S4.

To plot the trees, we used sumphycoeval to scale the branch length of all the sampled trees so that the posterior mean root age was 23.07 million years for Cyrtodactylus and 33.76 million years for Gekko. These ages are based on time-calibrated phylogenetic estimates from other data sets that are being prepared for publication. 


\section{Acknowledgments}

We thank Mark Holder for helpful advice with Hastings ratios and modeling the distribution on divergence times. This work was supported by funding provided to JRO from the National Science Foundation (NSF grant number DEB 1656004). The computational work was made possible by the Auburn University (AU) Hopper and Easley Clusters supported by the AU Office of Information Technology and a grant of high-performance computing resources and technical support from the Alabama Supercomputer Authority. Our gecko sampling was amassed with NSF support for fieldwork (EF-0334952, DEB 073199 and 0743491 to RMB; and 0804115 to CDS) and Fulbright grants to CDS. This paper is contribution number 949 of the Auburn University Museum of Natural History.

\section{References}

Barber, B. R. and J. Klicka. 2010. Two pulses of diversification across the Isthmus of Tehuantepec in a montane Mexican bird fauna. Proceedings Of The Royal Society B-Biological Sciences 277:2675-2681.

Bell, E. T. 1934. Exponential numbers. American Mathematical Monthly 41:411-419.

Bezanson, J., A. Edelman, S. Karpinski, and V. B. Shah. 2017. Julia: A fresh approach to numerical computing. SIAM review 59:65-98.

Blackburn, D. C., D. P. Bickford, A. C. Diesmos, D. T. Iskandar, and R. M. Brown. 2010. An ancient origin for the enigmatic flat-headed frogs (Bombinatoridae: Barbourula) from the Islands of Southeast Asia. PLoS ONE 5:10.

Breloff, T. 2021. Plots: Powerful convenience for Julia visualizations and data analysis. GitHub and archived on Zenodo.

Brooks, S. P. and A. Gelman. 1998. General methods for monitoring convergence of iterative simulations. Journal of Computational and Graphical Statistics 7:434-455.

Brown, R. M. 2016. Biogeography of land vertebrates. Pages 211-220 in Encyclopdia of Evolutionary Biology (R. M. Kilman, ed.) vol. 1 1st ed. Academic Press, Oxford, UK.

Brown, R. M. and A. C. Diesmos. 2009. Philippines, biology. Pages 723-732 in Encyclopdia of Islands (R. Gillespie and D. Clague, eds.). University of California Press, Berkeley.

Brown, R. M. and S. I. Guttman. 2002. Phylogenetic systematics of the Rana signata complex of Philippine and Bornean stream frogs: reconsideration of Huxley's modification of Wallace's line at the Oriental-Australian faunal zone interface. Biological Journal of the Linnean Society 76:393-461.

Brown, R. M. and C. D. Siler. 2014. Spotted stream frog diversification at the Australasian faunal zone interface, mainland versus island comparisons, and a test of the Philippine 'dual-umbilicus' hypothesis. Journal of Biogeography 41:182-195. 
Brown, R. M., C. D. Siler, C. H. Oliveros, J. A. Esselstyn, A. C. Diesmos, P. A. Hosner, C. W. Linkem, A. J. Barley, J. R. Oaks, M. B. Sanguila, L. J. Welton, R. G. Moyle, A. T. Peterson, and A. C. Alcala. 2013. Evolutionary processes of diversification in a model island archipelago. Annual Review of Ecology, Evolution, and Systematics 44:411-435.

Brown, R. M., C. D. Siler, S. J. Richards, A. C. Diesmos, and D. C. Cannatella. 2015. Multilocus phylogeny and a new classification for Southeast Asian and Melanesian forest frogs (family Ceratobatrachidae). Zoological Journal of the Linnean Society 174:130-168.

Brown, R. M., Y.-C. Su, B. Barger, C. D. Siler, M. B. Sanguila, A. C. Diesmos, and D. C. Blackburn. 2016. Phylogeny of the island archipelago frog genus Sanguirana: Another endemic Philippine radiation that diversified 'out-of-Palawan'. Molecular Phylogenetics and Evolution 94:531-536.

Bryant, D., R. Bouckaert, J. Felsenstein, N. A. Rosenberg, and A. Roychoudhury. 2012. Inferring species trees directly from biallelic genetic markers: Bypassing gene trees in a full coalescent analysis. Molecular Biology and Evolution 29:1917-1932.

Carlsson, K. and T. K. Papp. 2021. PGFPlotsX: a Julia package to generate publication quality figures using the LaTeX library PGFPlots. GitHub.

Catibog-Sinha, C. S. and L. R. Heaney, eds. 2006. Philippine Biodiversity: Principles and Practice. Haribon Foundation, Quezon City, Philippines.

Chan, K. O. and R. M. Brown. 2017. Did true frogs 'dispersify'? Biology Letters 13:20170299.

Chifman, J. and L. Kubatko. 2014. Quartet inference from SNP data under the coalescent model. Bioinformatics 30:3317-3324.

Clark, J. W. and P. C. J. Donoghue. 2017. Constraining the timing of whole genome duplication in plant evolutionary history. Proceedings of the Royal Society B: Biological Sciences 284:20170912.

Daza, J. M., T. A. Castoe, and C. L. Parkinson. 2010. Using regional comparative phylogeographic data from snake lineages to infer historical processes in Middle America. Ecography 33:343-354.

Diamond, J. M. and M. E. Gilpin. 1983. Biogeographic umbilici and the origin of the Philippine avifauna. Oikos 41:307-321.

Dickerson, R. E. 1928. Distribution of life in the Philippines. Philippine Bureau of Science, Manila, Philippines.

Doyle, J. J. and A. N. Egan. 2010. Dating the origins of polyploidy events. New Phytologist 186:73-85.

Drummond, A. J., S. Y. W. Ho, M. J. Phillips, and A. Rambaut. 2006. Relaxed phylogenetics and dating with confidence. PLoS Biology 4:e88. 
Drummond, A. J. and M. A. Suchard. 2010. Bayesian random local clocks, or one rate to rule them all. BMC Biology 8:114.

Eaton, D. A. R. and I. Overcast. 2020. ipyrad: Interactive assembly and analysis of RADseq datasets. Bioinformatics 36:2592-2594.

Evans, B., R. Brown, J. Mcguire, J. Supriatna, N. Andayani, A. Diesmos, D. Iskandar, D. Melnick, and D. Cannatella. 2003. Phylogenetics of fanged frogs: Testing biogeographical hypotheses at the interface of the Asian and Australian faunal zones. Systematic Biology 52:794-819.

Felsenstein, J. 1978. The number of evolutionary trees. Systematic Biology 27:27-33.

Foster, P. G. 2004. Modeling compositional heterogeneity. Systematic Biology 53:485-495.

Foster, P. G. 2018. Gram version 4.0.0. http://gram.nhm.ac.uk/.

Gavryushkina, A., T. A. Heath, D. T. Ksepka, T. Stadler, D. Welch, and A. J. Drummond. 2016. Bayesian total-evidence dating reveals the recent crown radiation of penguins. Systematic Biology 66:57-73.

Gearty, W. 2021. deeptime: Plotting Tools for Anyone Working in Deep Time. R package version 0.0 .6 .

Gong, L. and J. M. Flegal. 2016. A practical sequential stopping rule for high-dimensional Markov chain Monte Carlo. Journal of Computational and Graphical Statistics 25:684700.

Green, P. J. 1995. Reversible jump Markov chain Monte Carlo computation and Bayesian model determination. Biometrika 82:711-732.

Haq, B. U., J. Hardenbol, and P. R. Vail. 1987. Chronology of fluctuating sea levels since the Triassic. Science 235:1156-1167.

Hastings, W. K. 1970. Monte Carlo sampling methods using Markov chains and their applications. Biometrika 57:97-109.

Heaney, L. R. 1985. Zoogeographic evidence for middle and late pleistocene land bridges to the philippine islands. Mod Quatern Res SE Asia 9:127-144.

Heaney, L. R., D. S. Balete, E. A. Rickart, P. A. Alviola, M. R. M. Duya, M. V. Duya, M. J. Veluz, L. VandeVrede, and S. J. Steppan. 2011. Chapter 1: Seven new species and a new subgenus of forest mice (Rodentia: Muridae: Apomys) from Luzon Island. Fieldiana Life and Earth Sciences 2:1-60.

Heaney, L. R. and J. C. Regalado, Jr. 1998. Vanishing treasures of the Philippine rain forest. Field Museum, Chicago, Illinois. 
Heaney, L. R., J. S. Walsh, and A. T. Peterson. 2005. The roles of geological history and colonization abilities in genetic differentiation between mammalian populations in the Philippine Archipelago. Journal of Biogeography 32:229-247.

Heath, T. A., M. T. Holder, and J. P. Huelsenbeck. 2011. A Dirichlet process prior for estimating lineage-specific substitution rates. Molecular Biology and Evolution 29:939955.

Heath, T. A., J. P. Huelsenbeck, and T. Stadler. 2014. The fossilized birth-death process: A coherent model of fossil calibration for divergence time estimation. Proceedings of the National Academy of Sciences 111:E2957-E2966.

Heled, J. and A. J. Drummond. 2010. Bayesian inference of species trees from multilocus data. Molecular Biology and Evolution 27:570-580.

Hickerson, M. J., E. A. Stahl, and H. A. Lessios. 2006. Test for simultaneous divergence using approximate Bayesian computation. Evolution 60:2435-2453.

Hoelzer, G. A. and D. J. Meinick. 1994. Patterns of speciation and limits to phylogenetic resolution. Trends in Ecology \& Evolution 9:104-107.

Hosner, P. A., A. S. Nyári, and R. G. Moyle. 2013. Water barriers and intra-island isolation contribute to diversification in the insular Aethopyga sunbirds (Aves: Nectariniidae). Journal of Biogeography 40:1094-1106.

Huang, W., N. Takebayashi, Y. Qi, and M. J. Hickerson. 2011. MTML-msBayes: Approximate Bayesian comparative phylogeographic inference from multiple taxa and multiple loci with rate heterogeneity. BMC Bioinformatics 12:1.

Huxley, T. H. 1868. On the classification and the distribution of the Alectoromorphae and Heteromorphae. Proceedings of the Zoological Society of London 6:249-319.

Inger, R. F. 1954. Systematics and zoogeography of Philippine Amphibia. Fieldiana 33:182531.

Jansa, S. A., F. K. Barker, and L. R. Heaney. 2006. The pattern and timing of diversification of Philippine endemic rodents: Evidence from mitochondrial and nuclear gene sequences. Systematic Biology 55:73-88.

Jiao, Y., N. J. Wickett, S. Ayyampalayam, A. S. Chanderbali, L. Landherr, P. E. Ralph, L. P. Tomsho, Y. Hu, H. Liang, P. S. Soltis, D. E. Soltis, S. W. Clifton, S. E. Schlarbaum, S. C. Schuster, H. Ma, J. Leebens-Mack, and C. W. dePamphilis. 2011. Ancestral polyploidy in seed plants and angiosperms. Nature 473:97-100.

Jukes, T. H. and C. R. Cantor. 1969. Evolution of protein molecules. chap. 24, Pages 21-132 in Mammalian Protein Metabolism (H. N. Munro, ed.) vol. III. Academic Press, New York. 
Justiniano, R., J. J. Schenk, D. S. Balete, E. A. Rickart, J. A. Esselstyn, L. R. Heaney, and S. J. Steppan. 2015. Testing diversification models of endemic Philippine forest mice (Apomys) with nuclear phylogenies across elevational gradients reveals repeated colonization of isolated mountain ranges. Journal of Biogeography 42:51-64.

Kingman, J. F. C. 1982a. The coalescent. Stochastic processes and their applications 13:235248.

Kingman, J. F. C. 1982b. On the genealogy of large populations. Journal of Applied Probability 19:27-43.

Kishino, H., J. L. Thorne, and W. J. Bruno. 2001. Performance of a divergence time estimation method under a probabilistic model of rate evolution. Molecular Biology and Evolution 18:352-361.

Klinkenberg, D., J. A. Backer, X. Didelot, C. Colijn, and J. Wallinga. 2017. Simultaneous inference of phylogenetic and transmission trees in infectious disease outbreaks. PLOS Computational Biology 13:1-32.

Kuhner, M. K. and J. Felsenstein. 1994. A simulation comparison of phylogeny algorithms under equal and unequal evolutionary rates (erratum in mol. biol. evol. 1995; 12, 525). Molecular Biology and Evolution 11:459-468.

Lakner, C., P. van der Mark, J. P. Huelsenbeck, B. Larget, and F. Ronquist. 2008. Efficiency of Markov chain Monte Carlo tree proposals in Bayesian phylogenetics. Systematic Biology 57:86-103.

Leaché, A. D., S. C. Crews, and M. J. Hickerson. 2007. Two waves of diversification in mammals and reptiles of Baja California revealed by hierarchical Bayesian analysis. Biology Letters 3:646-650.

Lewis, P. O., M. T. Holder, and K. E. Holsinger. 2005. Polytomies and Bayesian phylogenetic inference. Systematic Biology 54:241-253.

Li, Z., G. P. Tiley, S. R. Galuska, C. R. Reardon, T. I. Kidder, R. J. Rundell, and M. S. Barker. 2018. Multiple large-scale gene and genome duplications during the evolution of hexapods. Proceedings of the National Academy of Sciences 115:4713-4718.

Linkem, C. W., A. C. Diesmos, and R. M. Brown. 2011. Molecular systematics of the Philippine forest skinks (Squamata: Scincidae: Sphenomorphus): testing morphological hypotheses of interspecific relationships. Zoological Journal of the Linnean Society 163:1217-1243.

Linkem, C. W., K. M. Hesed, A. C. Diesmos, and R. M. Brown. 2010. Species boundaries and cryptic lineage diversity in a Philippine forest skink complex (Reptilia; Squamata; Scincidae: Lygosominae). Molecular Phylogenetics and Evolution 56:572-585. 
Liu, L. and D. K. Pearl. 2007. Species trees from gene trees: Reconstructing Bayesian posterior distributions of a species phylogeny using estimatated gene tree distributions. Systematic Biology 56:504-514.

Lomolino, M. V., B. R. Riddle, and J. H. Brown. 2016. Biogeography. 5th ed. Sinauer Associates, Sunderland, Massachusetts, USA.

Mann, H. B. and D. R. Whitney. 1947. On a test of whether one of two random variables is stochastically larger than the other. The Annals of Mathematical Statistics 18:50-60.

Metropolis, N., A. W. Rosenbluth, M. N. Rosenbluth, A. H. Teller, and E. Teller. 1953. Equation of state calculations by fast computing machines. The Journal of Chemical Physics $21: 1087-1092$.

Miller, K. G., M. A. Kominz, J. V. Browning, J. D. Wright, G. S. Mountain, M. E. Katz, P. J. Sugarman, B. S. Cramer, N. Christie-Blick, and S. F. Pekar. 2005. The Phanerozoic record of global sea-level change. Science 310:1293-1298.

Nielsen, R. and J. Wakeley. 2001. Distinguishing migration from isolation: A Markov chain Monte Carlo approach. Genetics 158:885-896.

Oaks, J. R. 2014. An improved approximate-bayesian model-choice method for estimating shared evolutionary history. BMC Evolutionary Biology 14:150.

Oaks, J. R. 2019. Full Bayesian comparative phylogeography from genomic data. Systematic Biology 68:371-395.

Oaks, J. R. 2021. Analyses exploring the behavior of generalized bayesian phylogenetics: Version 1.0.0. GitHub and archived on Zenodo; https://doi.org/10.5281/zenodo. 5162056.

Oaks, J. R., N. L'Bahy, and K. A. Cobb. 2020. Insights from a general, full-likelihood Bayesian approach to inferring shared evolutionary events from genomic data: Inferring shared demographic events is challenging. Evolution 74:2184-2206.

Oaks, J. R., C. D. Siler, and R. M. Brown. 2019. The comparative biogeography of Philippine geckos challenges predictions from a paradigm of climate-driven vicariant diversification across an island archipelago. Evolution 73:1151-1167.

Oaks, J. R., J. Sukumaran, J. A. Esselstyn, C. W. Linkem, C. D. Siler, M. T. Holder, and R. M. Brown. 2013. Evidence for climate-driven diversification? a caution for interpreting ABC inferences of simultaneous historical events. Evolution 67:991-1010.

Oaks, J. R. and P. L. Wood, Jr. 2021. Open-science notebook for the comparative phylogenetics of philippine gekkonids: Version 2. GitHub and archived on Zenodo; https: //doi.org/10.5281/zenodo. 5162085. 
Plouviez, S., T. M. Shank, B. Faure, C. Daguin-Thiebaut, F. Viard, F. H. Lallier, and D. Jollivet. 2009. Comparative phylogeography among hydrothermal vent species along the East Pacific Rise reveals vicariant processes and population expansion in the South. Molecular Ecology 18:3903-3917.

Pybus, O. G. and A. Rambaut. 2009. Evolutionary analysis of the dynamics of viral infectious disease. Nature Reviews Genetics 10:540-550.

Rannala, B. and Z. Yang. 2003. Bayes estimation of species divergence times and ancestral population sizes using DNA sequences from multiple loci. Genetics 164:1645-1656.

Roberts, T. E. 2006. Multiple levels of allopatric divergence in the endemic Philippine fruit bat Haplonycteris fischeri (Pteropodidae). Biological Journal of the Linnean Society 88:329-349.

Robinson, D. F. and L. R. Foulds. 1979. Comparison of weighted labelled trees. Pages 119126 in Combinatorial Mathematics VI (A. F. Horadam and W. D. Wallis, eds.) Springer Berlin Heidelberg, Berlin, Heidelberg.

Rohling, E. J., M. Fenton, F. J. Jorissen, P. Bertrand, G. Ganssen, and J. P. Caulet. 1998. Magnitudes of sea-level lowstands of the past 500,000 years. Nature 394:162-165.

Siddall, M., E. J. Rohling, A. Almogi-Labin, C. Hemleben, D. Meischner, I. Schmelzer, and D. A. Smeed. 2003. Sea-level fluctuations during the last glacial cycle. Nature 423:853-858.

Siler, C. D., A. C. Diesmos, A. C. Alcala, and R. M. Brown. 2011. Phylogeny of Philippine slender skinks (Scincidae: Brachymeles) reveals underestimated species diversity, complex biogeographical relationships, and cryptic patterns of lineage diversification. Molecular Phylogenetics and Evolution 59:53-65.

Siler, C. D., J. R. Oaks, K. Cobb, O. Hidetoshi, and R. M. Brown. 2014. Critically endangered island endemic or peripheral population of a widespread species? conservation genetics of Kikuchi's gecko and the global challenge of protecting peripheral oceanic island endemic vertebrates. Diversity and Distributions 20:756-772.

Siler, C. D., J. R. Oaks, J. A. Esselstyn, A. C. Diesmos, and R. M. Brown. 2010. Phylogeny and biogeography of Philippine bent-toed geckos (Gekkonidae: Cyrtodactylus) contradict a prevailing model of Pleistocene diversification. Molecular Phylogenetics and Evolution 55:699-710.

Siler, C. D., J. R. Oaks, L. J. Welton, C. W. Linkem, J. Swab, A. C. Diesmos, and R. M. Brown. 2012. Did geckos ride the Palawan raft to the Philippines? Journal of Biogeography 39:1217-1234.

Slowikowski, K. 2020. ggrepel: Automatically Position Non-Overlapping Text Labels with ggplot2. R package ggrepel version 0.9.1.

Spratt, R. M. and L. E. Lisiecki. 2016. A Late Pleistocene sea level stack. Climate of the Past 12:1079-1092. 
Stadler, T. 2010. Sampling-through-time in birth-death trees. Journal of Theoretical Biology $267: 396-404$.

Stadler, T., A. Gavryushkina, R. C. Warnock, A. J. Drummond, and T. A. Heath. 2018. The fossilized birth-death model for the analysis of stratigraphic range data under different speciation modes. Journal of Theoretical Biology 447:41-55.

Suchard, M. A., R. E. Weiss, and J. S. Sinsheimer. 2001. Bayesian selection of continuoustime Markov chain evolutionary models. Molecular Biology And Evolution 18:1001-1013.

Suzuki, Y., G. V. Glazko, and M. Nei. 2002. Overcredibility of molecular phylogenies obtained by bayesian phylogenetics. Proceedings of the National Academy of Sciences 99:1613816143.

Voje, K. L., C. Hemp, Ø. Flagstad, G.-P. Saetre, and N. C. Stenseth. 2009. Climatic change as an engine for speciation in flightless Orthoptera species inhabiting African mountains. Molecular Ecology 18:93-108.

Wallace, A. R. 1869. The Malay Archpelago: The Land of the Orang-utan, and the Bird of Paradise. Macmillan and Co., London.

Wang, L.-G., T. T.-Y. Lam, S. Xu, Z. Dai, L. Zhou, T. Feng, P. Guo, C. W. Dunn, B. R. Jones, T. Bradley, H. Zhu, Y. Guan, Y. Jiang, and G. Yu. 2019. Treeio: An R package for phylogenetic tree input and output with richly annotated and associated data. Molecular Biology and Evolution 37:599-603.

Wickham, H. 2016. ggplot2: Elegant Graphics for Data Analysis. Springer-Verlag New York.

Wilcoxon, F. 1945. Individual comparisons by ranking methods. Biometrics Bulletin 1:80-83.

Wilke, C. O. 2020. cowplot: Streamlined Plot Theme and Plot Annotations for ggplot2. R package version 1.1.1.

Wood, Jr., P. L., X. Guo, S. L. Travers, Y.-C. Su, K. V. Olson, A. M. Bauer, L. L. Grismer, C. D. Siler, R. G. Moyle, M. J. Andersen, and R. M. Brown. 2020. Parachute geckos free fall into synonymy: Gekko phylogeny, and a new subgeneric classification, inferred from thousands of ultraconserved elements. Molecular Phylogenetics and Evolution 146:106731.

Yang, Z. 1994. Statistical properties of the maximum likelihood method of phylogenetic estimation and comparison with distance matrix methods. Systematic Biology 43:329342.

Yang, Z. 2014. Molecular Evolution: A Statistical Approach. Oxford University Press, Oxford, United Kingdom.

Yang, Z., N. Goldman, and A. Friday. 1995. Maximum likelihood trees from DNA sequences: A peculiar statistical estimation problem. Systematic Biology 44:384-399.

Ypma, R. J. F., W. M. van Ballegooijen, and J. Wallinga. 2013. Relating phylogenetic trees to transmission trees of infectious disease outbreaks. Genetics 195:1055-1062. 
Yu, G., D. K. Smith, H. Zhu, Y. Guan, and T. T.-Y. Lam. 2017. ggtree: an R package for visualization and annotation of phylogenetic trees with their covariates and other associated data. Methods in Ecology and Evolution 8:28-36. 


\section{Supporting Information}

Title: Generalizing Bayesian phylogenetics to infer shared evolutionary events

Authors: Jamie R. Oaks Corresponding author: joaks@auburn.edu ${ }^{1}$, Perry L. Wood, Jr. ${ }^{1}$, Cameron D. Siler ${ }^{2}$, and Rafe M. Brown ${ }^{3}$

${ }^{1}$ Department of Biological Sciences \& Museum of Natural History, Auburn University, Auburn, Alabama 36849

${ }^{2}$ Sam Noble Oklahoma Museum of Natural History and Department of Biology, University of Oklahoma, Norman, Oklahoma 73072-7029

${ }^{3}$ Biodiversity Institute and Department of Ecology and Evolutionary Biology, University of Kansas, Lawrence, Kansas 66045, USA

\section{Table of Contents}

1 Figures referenced in main text

2 Tables

3 The generalized tree model

4 Approximating the posterior of the generalized tree model $\quad 17$

4.1 Split-time move . . . . . . . . . . . . . . . . . . 17

4.1 .1 Drawing the new divergence time . . . . . . . . . . . 17

$4.1 .2 \quad$ Prior ratio . . . . . . . . . . . . . . . . . . . 18

4.1 .3 Hastings ratio . . . . . . . . . . . . . . . . . 18

4.2 Merge-times move . . . . . . . . . . . . . . . . . . . . 19

4.2 .1 Prior ratio . . . . . . . . . . . . . . . . . 19

4.2 .2 Hastings ratio . . . . . . . . . . . . . . . . 20

4.3 Expanding $\Xi \ldots \ldots \ldots \ldots \ldots \ldots$

4.3.1 The case of all bifurcating nodes mapped to $\tau_{i} \ldots \ldots$. . . . . . . 21

4.3.2 The case of a single polytomy mapped to $\tau_{i} \ldots \ldots$. . . . . . 21

4.3.3 The case when multiple nodes, including at least one polytomy, is mapped to $\tau_{i} \ldots \ldots \ldots \ldots \ldots \ldots \ldots$

4.4 Validation of Split-time and Merge-times moves . . . . . . . . . . 23

4.5 Nested-neighbor-node-swap move . . . . . . . . . . . . . . . 24

4.5 .1 Validation of move . . . . . . . . . . . . . . . 25

4.6 Divergence time slide bump move . . . . . . . . . . . . . . . 26 
bioRxiv preprint doi: https://doi.org/10.1101/2021.07.23.453597; this version posted October 17, 2021. The copyright holder for this preprint (which was not certified by peer review) is the author/funder, who has granted bioRxiv a license to display the preprint in perpetuity. It is made available under aCC-BY 4.0 International license.

4.6.1 An extension to this move . . . . . . . . . . . . . . . 29

4.6.2 Validation of this move . . . . . . . . . . . . . . 30 
bioRxiv preprint doi: https://doi.org/10.1101/2021.07.23.453597; this version posted October 17, 2021. The copyright holder for this preprint (which was not certified by peer review) is the author/funder, who has granted bioRxiv a license to display the preprint in perpetuity. It is made available under aCC-BY 4.0 International license.

\section{Figures referenced in main text}


bioRxiv preprint doi: https://doi.org/10.1101/2021.07.23.453597; this version posted October 17,2021 . The copyright holder for this preprint (which was not certified by peer review) is the author/funder, who has granted bioRxiv a license to display the preprint in perpetuity. It is made available under aCC-BY 4.0 International license.

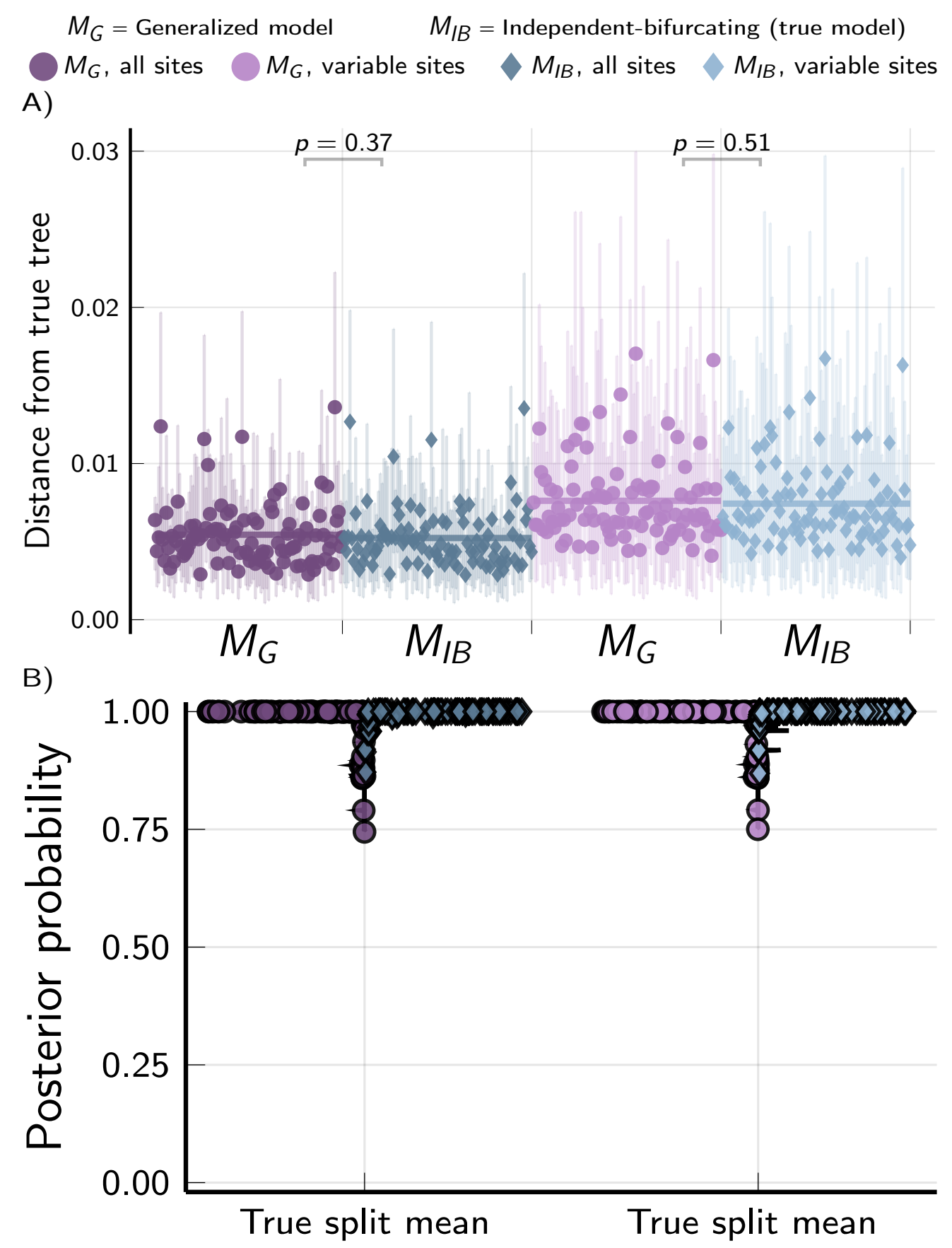

Figure S1. The performance of the $M_{G}$ and $M_{I B}$ tree models when applied to 100 data sets, each with 50,000 biallelic characters simulated on species trees randomly drawn from the $M_{I B}$ tree distribution. (A) The square root of the sum of squared differences in branch lengths between the true tree and each posterior tree sample (Kuhner and Felsenstein, 1994); the point and bars represent the posterior mean and equal-tailed 95\% credible interval, respectively. P-values are shown for Wilcoxon signed-rank tests (Wilcoxon, 1945) comparing the paired differences in tree distances between methods. (B) Violin plots comparing the mean posterior probabilities of true splits for each of the 100 simulated trees. For each simulation, the mutation-scaled effective population size $\left(N_{e} \mu\right)$ was drawn from a gamma distribution (shape $=20$, mean $\left.=0.001\right)$ and shared across all the branches of the tree; this distribution was used as the prior in analyses. Plots created using the PGFPlotsX (Version 1.2.10, Commit 1adde3d0; Carlsson and Papp, 2021) backend of the Plots (Version 1.5.7, Commit f80ce6a2; Breloff, 2021) package in Julia (Version 1.5.4; Bezanson et al., 2017). 
bioRxiv preprint doi: https://doi.org/10.1101/2021.07.23.453597; this version posted October 17, 2021. The copyright holder for this preprint (which was not certified by peer review) is the author/funder, who has granted bioRxiv a license to display the preprint in perpetuity. It is made available under aCC-BY 4.0 International license.

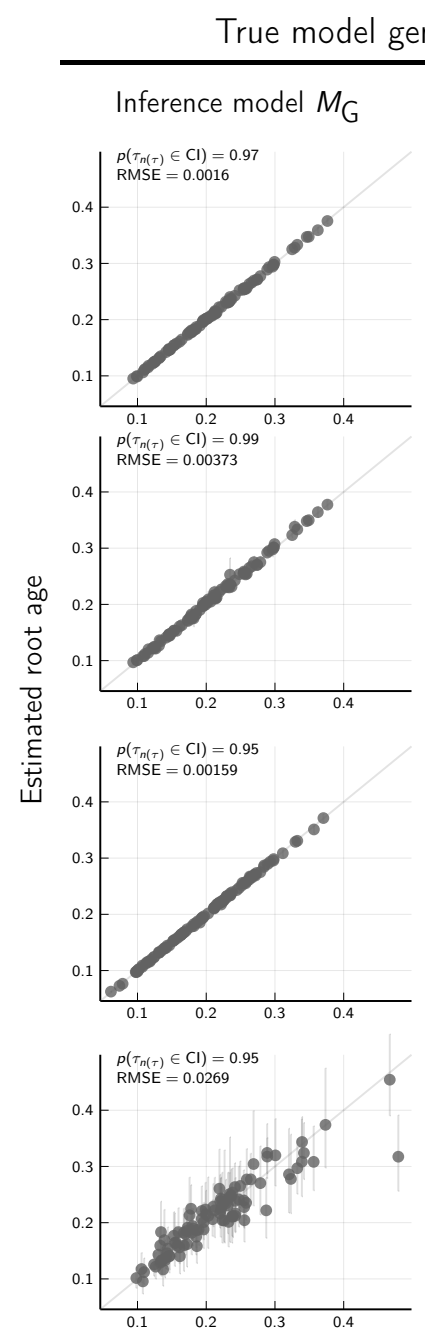

True model generalized $\left(M_{\mathrm{G}}\right)$

Inference model $M_{\mathrm{IB}}$
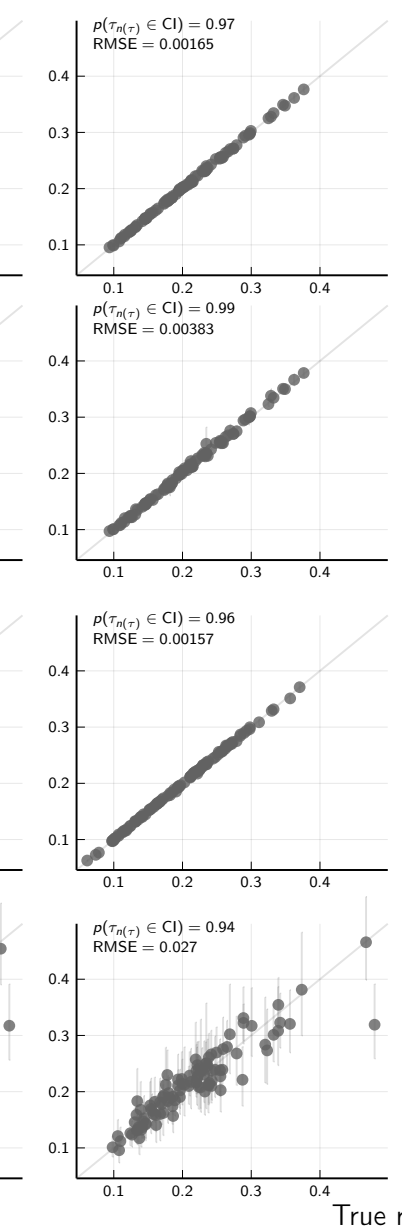

True model bifurcating \& independent $\left(M_{\mathrm{IB}}\right)$

$$
\text { Inference model } M_{\mathrm{G}} \quad \text { Inference model } M_{\mathrm{IB}}
$$
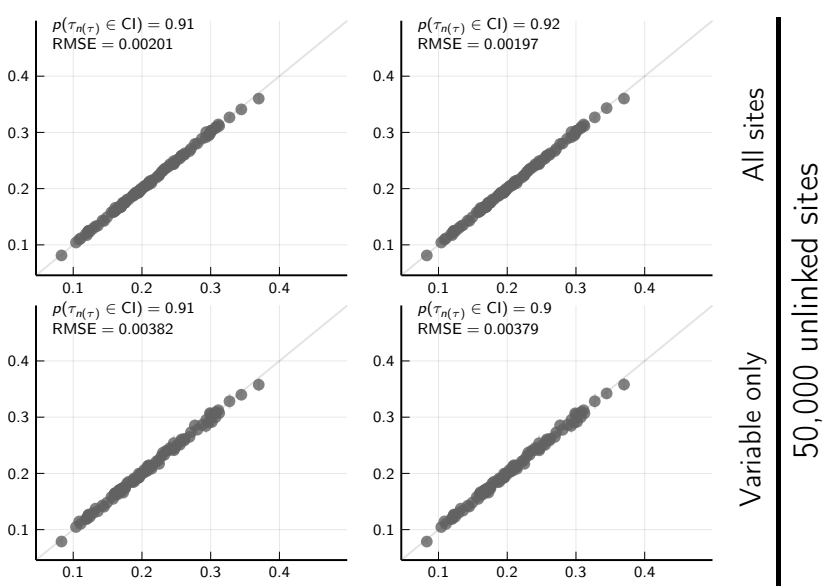

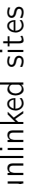

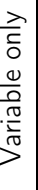

ते
$\frac{1}{0}$
$\frac{0}{0}$
$\frac{.0}{\frac{\pi}{2}}$
$>$
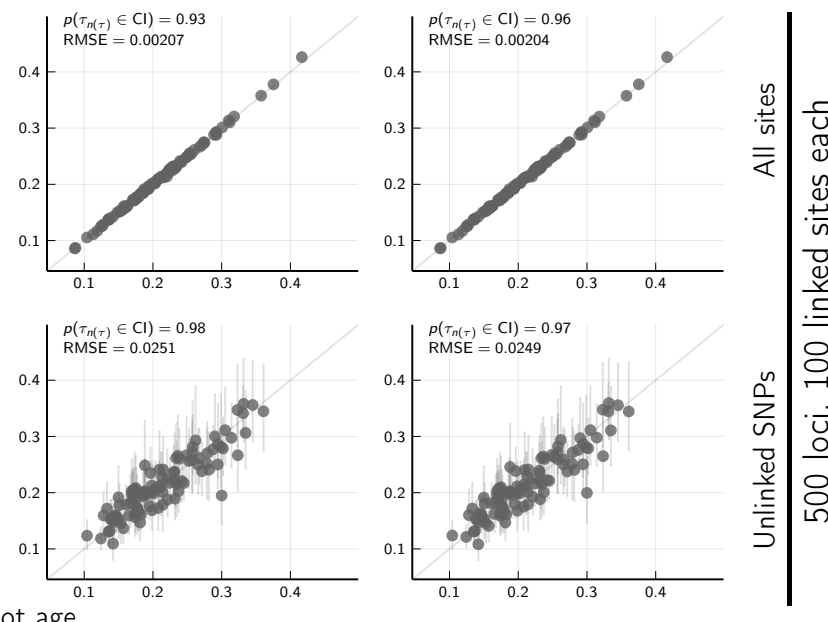

Figure S2. The accuracy and precision of the $M_{G}$ and $M_{I B}$ models at estimating the age of the root (in expected subsitutions per site) from data sets with 50,000 biallelic characters simulated on species trees randomly drawn from the $M_{G}$ and $M_{I B}$ tree distributions. Each plotted circle and associated error bars represent the posterior mean and $95 \%$ credible interval. Estimates for which the potential-scale reduction factor was greater than 1.2 (Brooks and Gelman, 1998) or the effective sample size was less than 200 are highlighted in red. Plots created using the PGFPlotsX (Version 1.2.10, Commit 1adde3d0; Carlsson and Papp, 2021) backend of the Plots (Version 1.5.7, Commit f80ce6a2; Breloff, 2021) package in Julia (Version 1.5.4; Bezanson et al., 2017). 
bioRxiv preprint doi: https://doi.org/10.1101/2021.07.23.453597; this version posted October 17, 2021. The copyright holder for this preprint (which was not certified by peer review) is the author/funder, who has granted bioRxiv a license to display the preprint in perpetuity. It is made available under aCC-BY 4.0 International license.

True model generalized $\left(M_{\mathrm{G}}\right)$
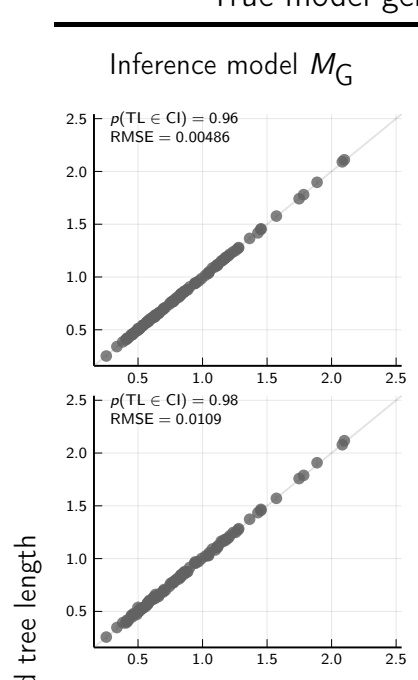

Inference model $M_{\mathrm{IB}}$
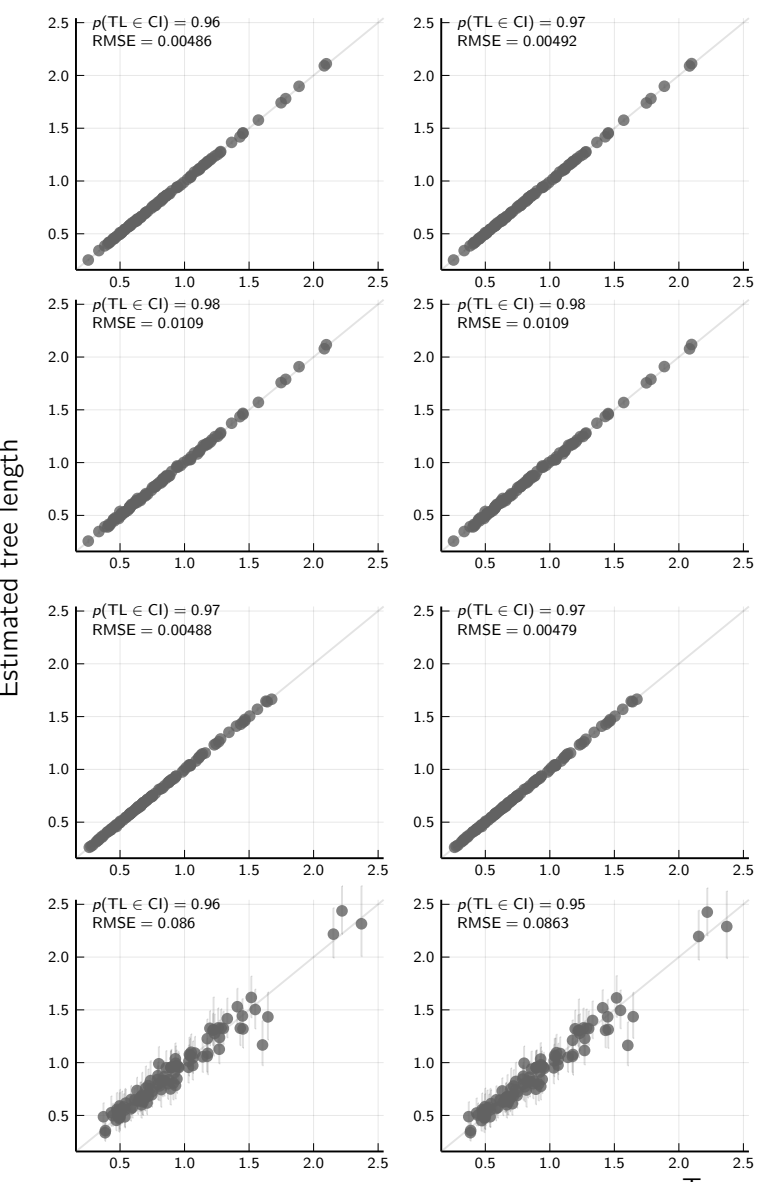
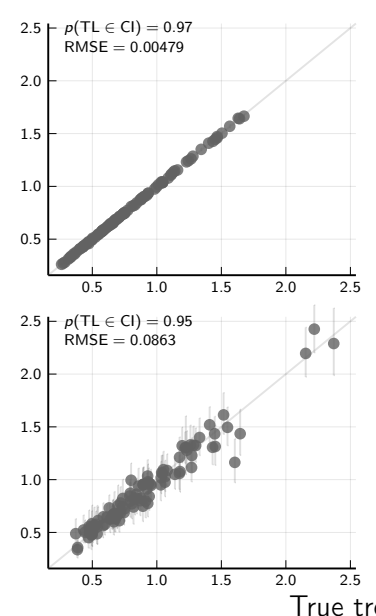

True model bifurcating \& independent $\left(M_{\mathrm{IB}}\right)$

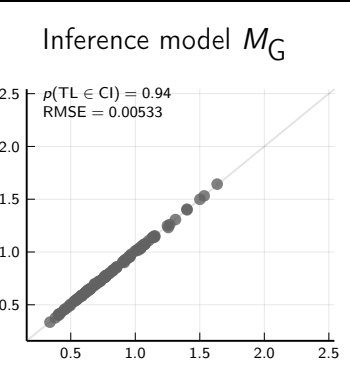

Inference model $M_{\mathrm{IB}}$
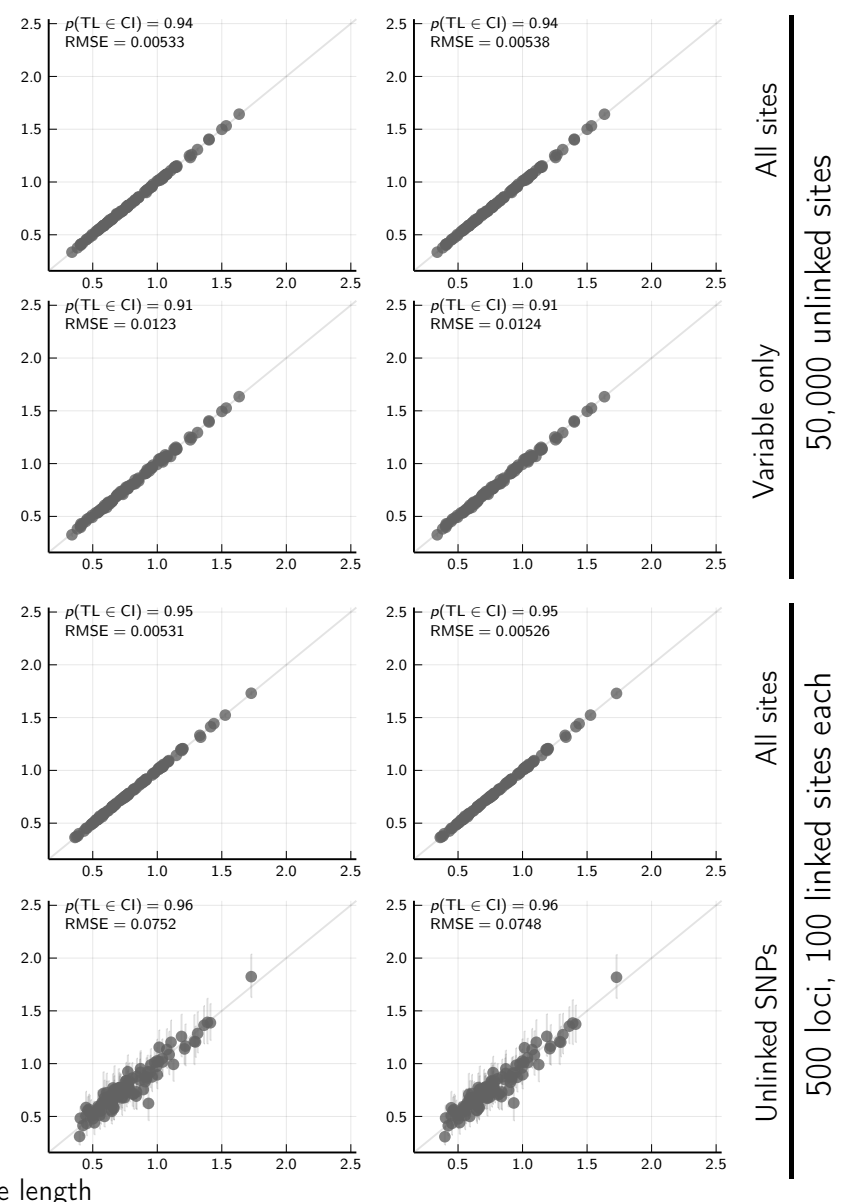

Figure S3. The accuracy and precision of the $M_{G}$ and $M_{I B}$ models at estimating the tree length (the sum of all branch lengths in units of expected substitutions per site) from data sets with 50,000 biallelic characters simulated on species trees randomly drawn from the $M_{G}$ and $M_{I B}$ tree distributions. Each plotted circle and associated error bars represent the posterior mean and 95\% credible interval. Estimates for which the potentialscale reduction factor was greater than 1.2 (Brooks and Gelman, 1998) or the effective sample size was less than 200 are highlighted in red. Plots created using the PGFPlotsX (Version 1.2.10, Commit 1adde3d0; Carlsson and Papp, 2021) backend of the Plots (Version 1.5.7, Commit f80ce6a2; Breloff, 2021) package in Julia (Version 1.5.4; Bezanson et al., 2017). 
bioRxiv preprint doi: https://doi.org/10.1101/2021.07.23.453597; this version posted October 17, 2021. The copyright holder for this preprint (which was not certified by peer review) is the author/funder, who has granted bioRxiv a license to display the preprint in perpetuity. It is made available under aCC-BY 4.0 International license.

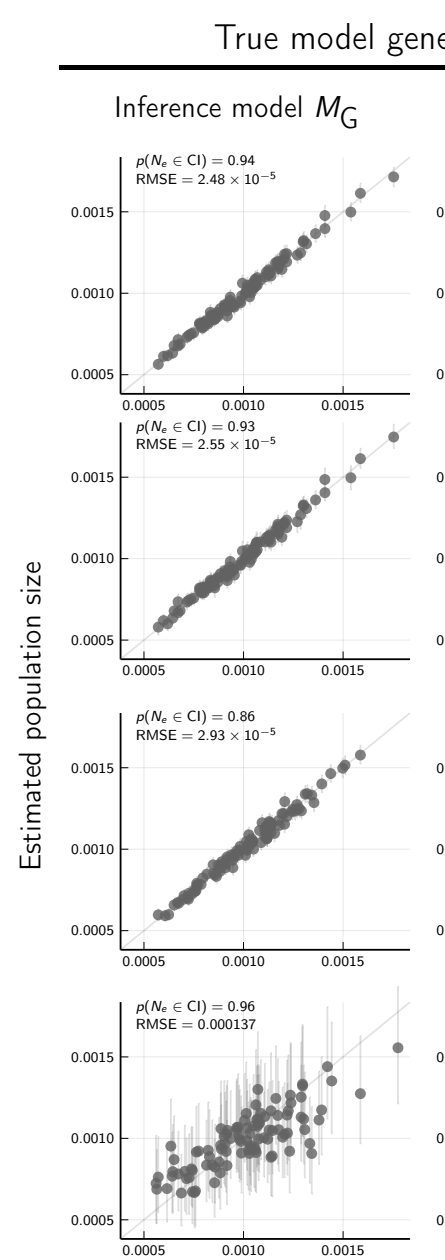

True model generalized $\left(M_{\mathrm{G}}\right)$

Inference model $M_{\mathrm{IB}}$
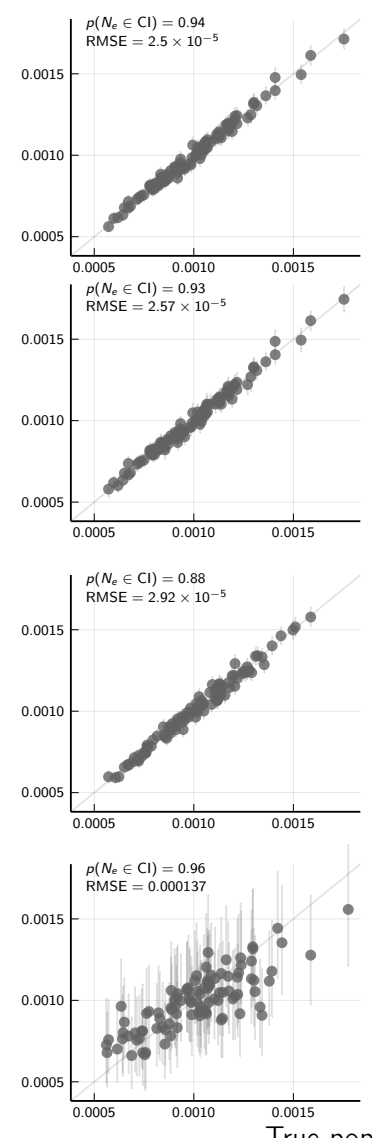

True model bifurcating \& independent $\left(M_{\mathrm{IB}}\right)$

Inference model $M_{\mathrm{G}} \quad$ Inference model $M_{\mathrm{IB}}$
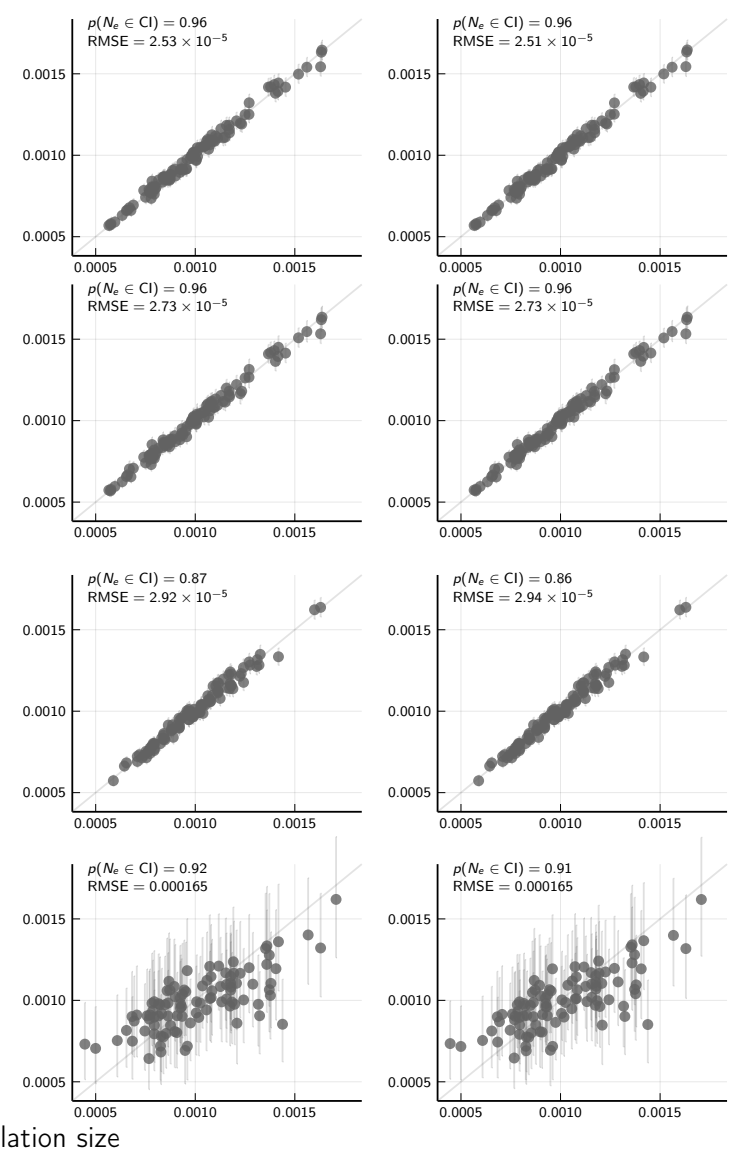

Figure S4. The accuracy and precision of the $M_{G}$ and $M_{I B}$ models at estimating the effective population size $\left(N_{e} \mu\right)$ across the tree from data sets with 50,000 biallelic characters simulated on species trees randomly drawn from the $M_{G}$ and $M_{I B}$ tree distributions. Each plotted circle and associated error bars represent the posterior mean and $95 \%$ credible interval. Estimates for which the potential-scale reduction factor was greater than 1.2 (Brooks and Gelman, 1998) or the effective sample size was less than 200 are highlighted in red. Plots created using the PGFPlotsX (Version 1.2.10, Commit 1adde3d0; Carlsson and Papp, 2021) backend of the Plots (Version 1.5.7, Commit f80ce6a2; Breloff, 2021) package in Julia (Version 1.5.4; Bezanson et al., 2017). 


\section{Probability of incorrectly merging neighboring div times \\ True model $=M_{\mathrm{IB}}$}
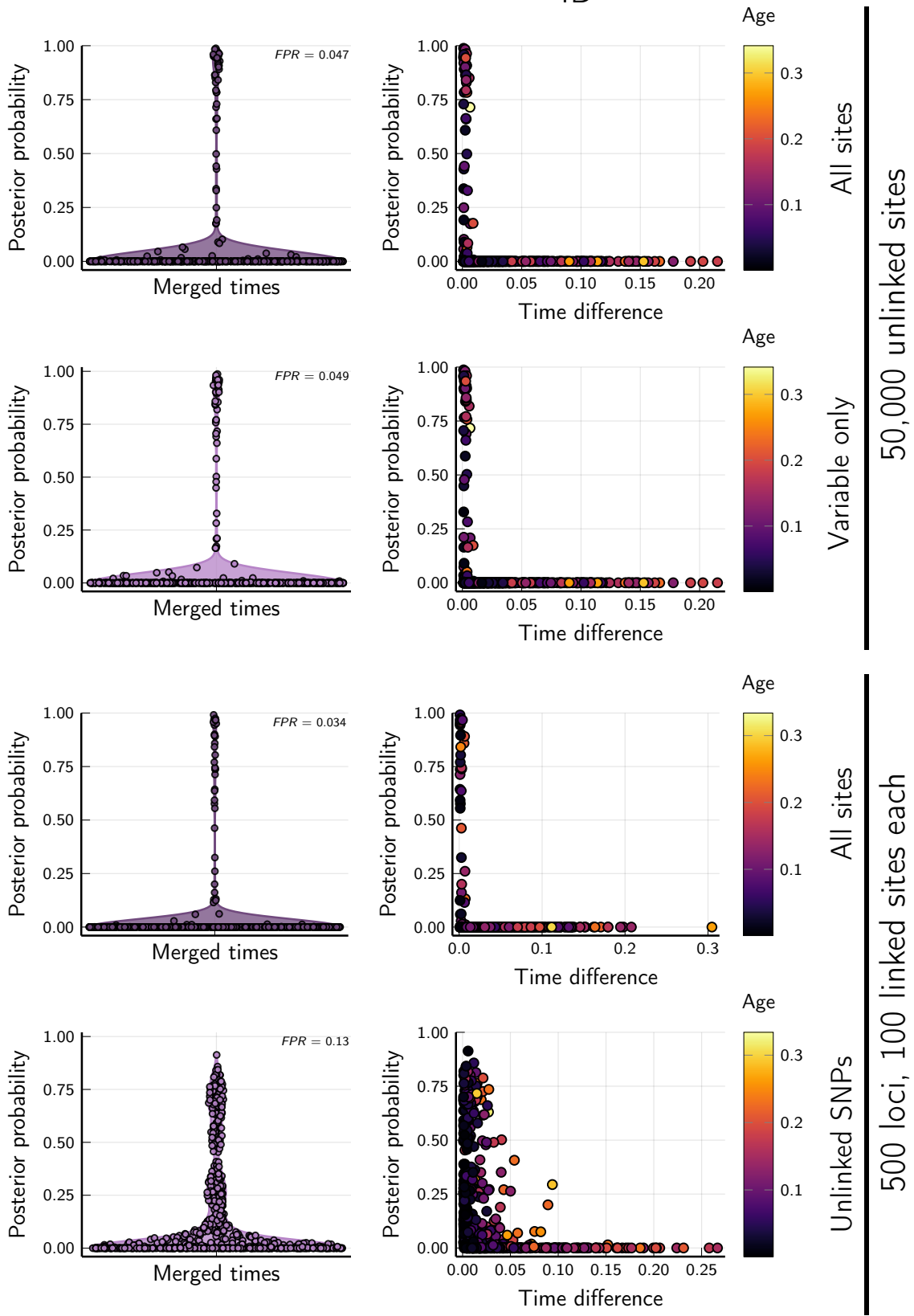

Figure S5. The $M_{G}$ model has a low false positive rate (FPR; the proportion of incorrectly merged divergence times with a posterior probability $>0.5$ ) when applied to data simulated on trees drawn from $M_{I B}$ (no shared or multifurcating divergences) with all (Row 1 ) or only variable (Row 2) unlinked characters, and all characters from linked loci (Row 3). Support for incorrectly merged divergence times is high only when the difference between the times is small (right, X-axis, units of expected substitutions per site), and is not correlated with the age of the merged nodes (right, color gradient in units of expected substitutions per site). When data sets with linked loci are reduced to only one variable site per locus (Row 4), the FPR increases (left) and precision decreases (right). The top row is the same as Figure 5. Plots created using the PGFPlotsX (Version 1.2.10, Commit 1adde3d0; Carlsson and Papp, 2021) backend of the Plots (Version 1.5.7, Commit f80ce6a2; Breloff, 2021) package in Julia (Version 1.5.4; Bezanson et al., 2017). 


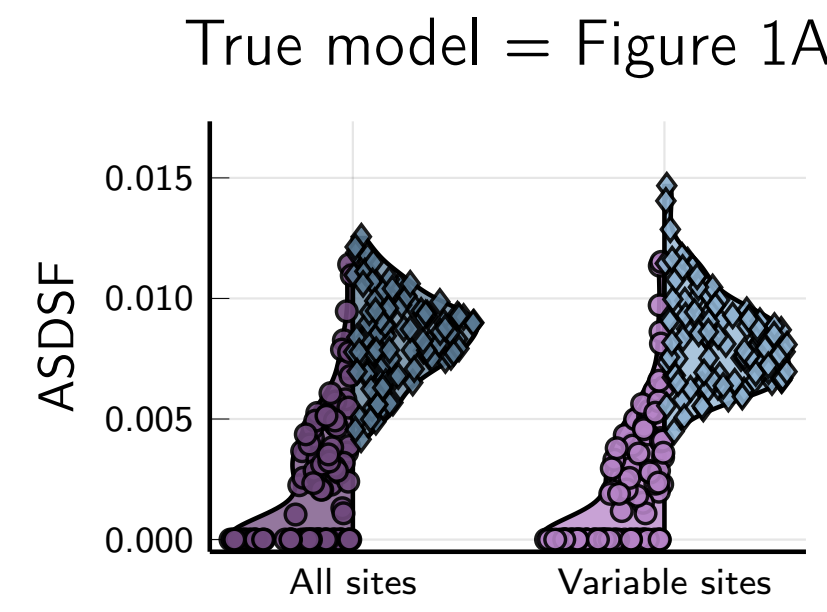

True model $=$ Figure $2 \mathrm{~A}$
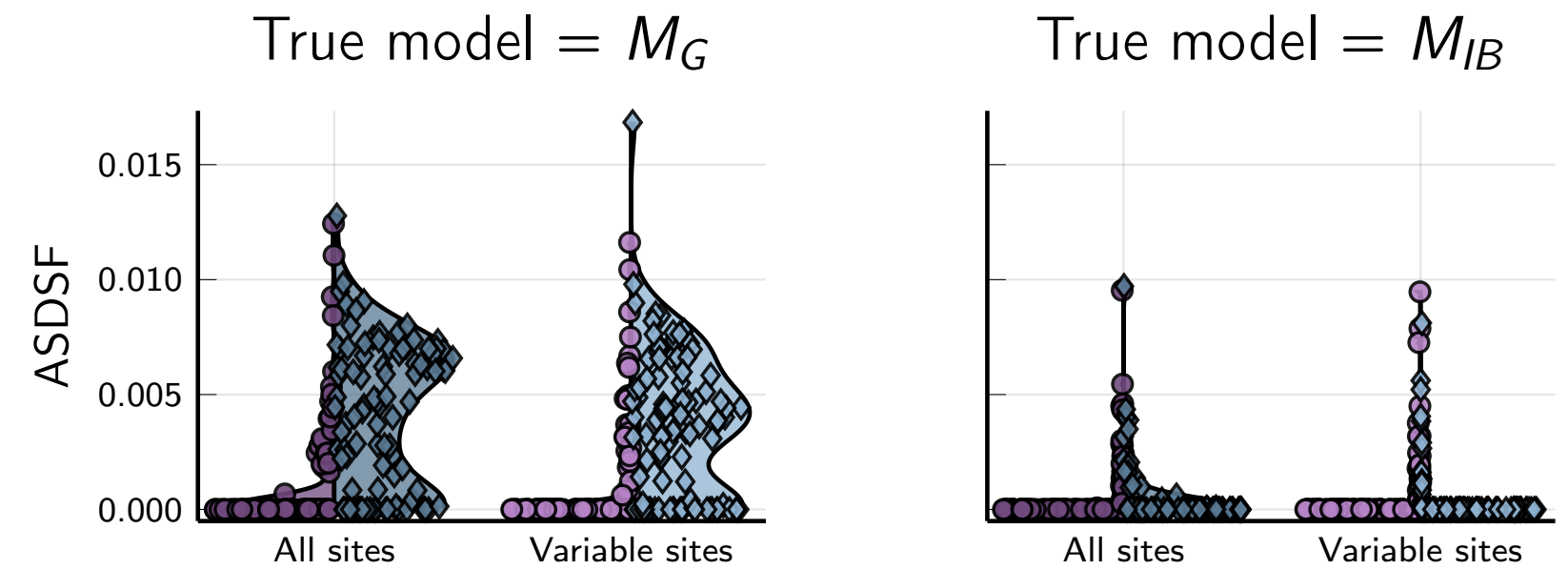

Figure S6. Markov chain Monte Carlo (MCMC) sampling yielded better convergence and mixing among chains under the generalized tree model when there were shared or multifurcating divergences. Plots created using the PGFPlotsX (Version 1.2.10, Commit 1adde3d0; Carlsson and Papp, 2021) backend of the Plots (Version 1.5.7, Commit f80ce6a2; Breloff, 2021) package in Julia (Version 1.5.4; Bezanson et al., 2017). 


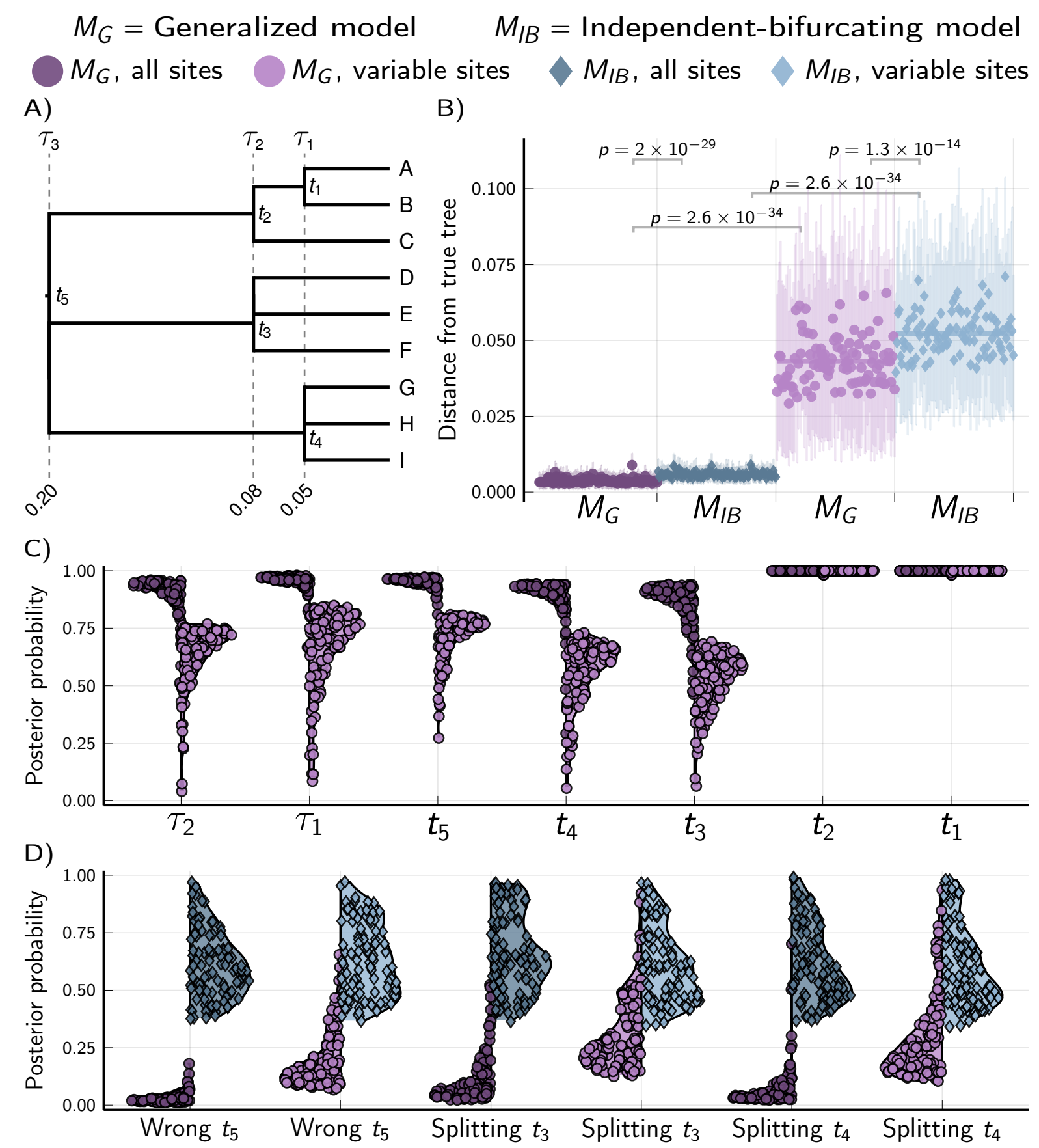

Figure S7. Results of analyses of 100 data sets with linked loci simulated along the species tree shown in (A). Each simulated data set comprised 500 loci with 100 biallelic characters. P-values are shown for Mann-Whitney U tests Mann and Whitney (1947) comparing the differences in tree distances between methods. For each simulation, the mutation-scaled effective population size $\left(N_{e} \mu\right)$ was drawn from a gamma distribution (shape $=20$, mean $=0.001)$ and shared across all the branches of the tree; this distribution was used as the prior in analyses. Tree plotted using Gram (Version 4.0.0, Commit 02286362; Foster, 2018) and the P4 phylogenetic toolkit (Version 1.4, Commit d9c8d1b1; Foster, 2004). Other plots created using the PGFPlotsX (Version 1.2.10, Commit 1adde3d0; Carlsson and Papp, 2021) backend of the Plots (Version 1.5.7, Commit f80ce6a2; Breloff, 2021) package in Julia (Version 1.5.4; Bezanson et al., 2017). 


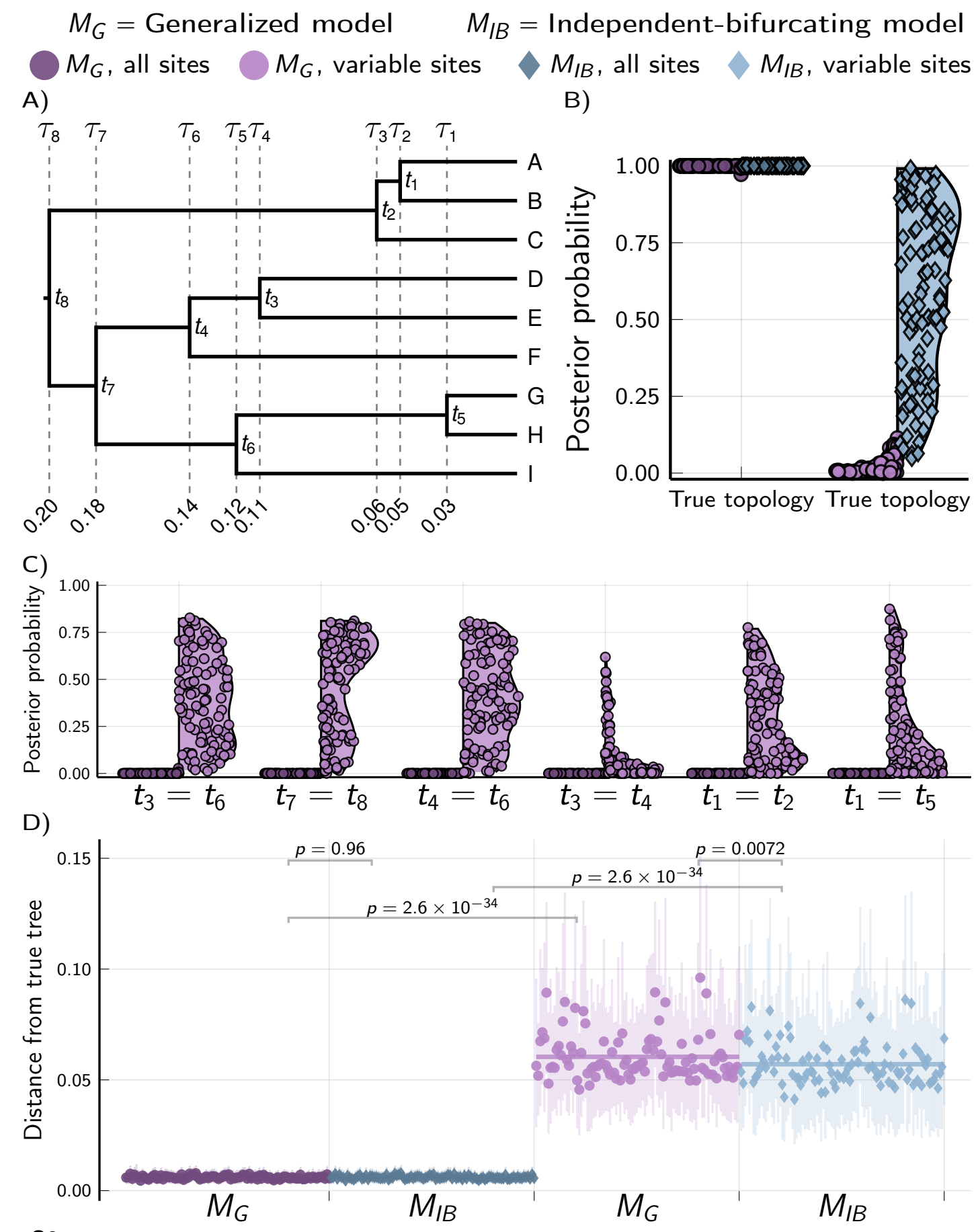

Figure S8. Results of analyses of 100 data sets with linked loci simulated along the species tree shown in (A). Each simulated data set comprised 500 loci with 100 biallelic characters. P-values are shown for Mann-Whitney $U$ tests (Mann and Whitney, 1947) comparing the differences in tree distances between methods. For each simulation, the mutation-scaled effective population size $\left(N_{e} \mu\right)$ was drawn from a gamma distribution (shape $=20$, mean $=0.001)$ and shared across all the branches of the tree; this distribution was used as the prior in analyses. Tree plotted using Gram (Version 4.0.0, Commit 02286362; Foster, 2018) and the P4 phylogenetic toolkit (Version 1.4, Commit d9c8d1b1; Foster, 2004). Other plots created using the PGFPlotsX (Version 1.2.10, Commit 1adde3d0; Carlsson and Papp, 2021) backend of the Plots (Version 1.5.7, Commit f80ce6a2; Breloff, 2021) package in Julia (Version 1.5.4; Bezanson et al., 2017). 

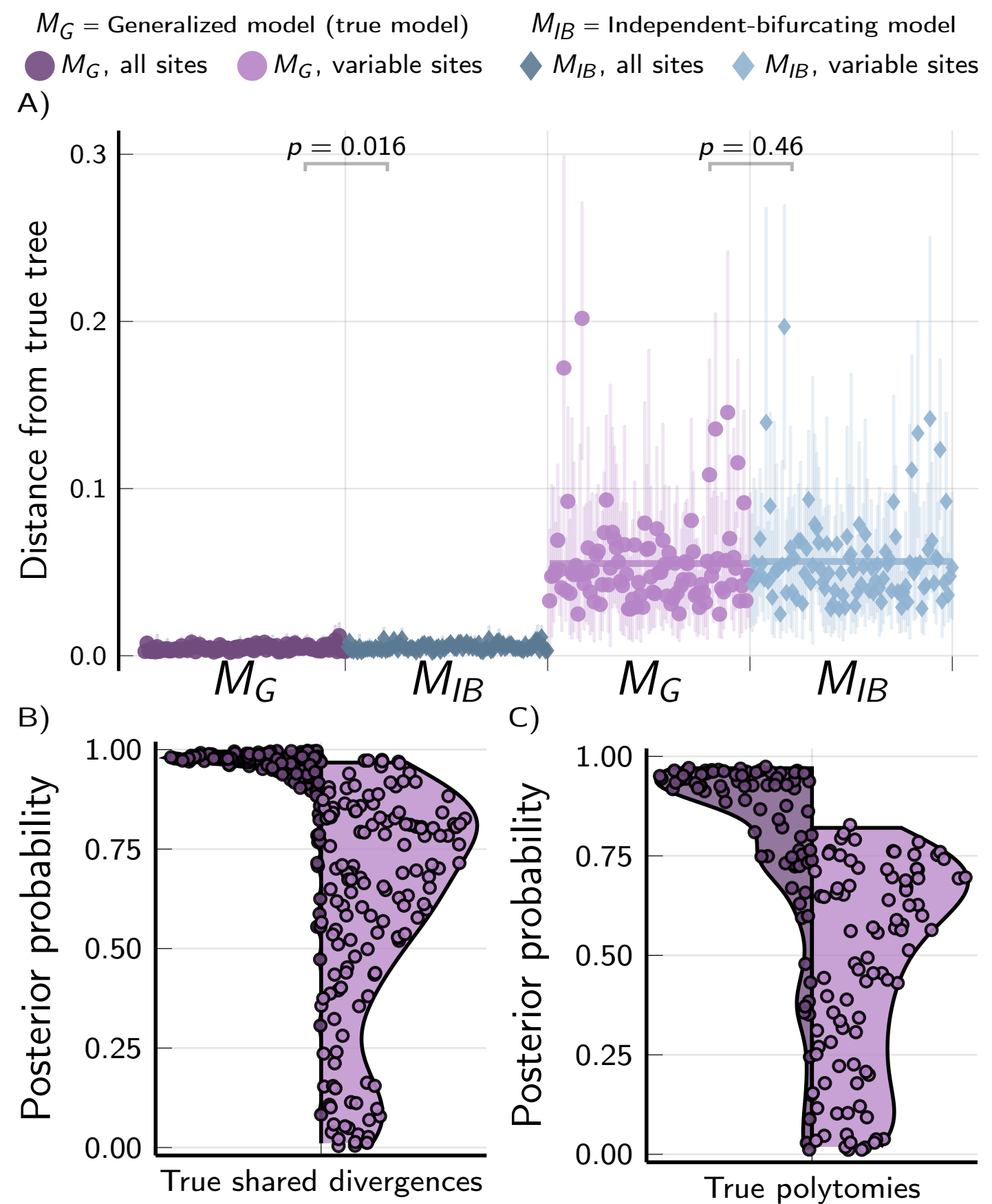

C)

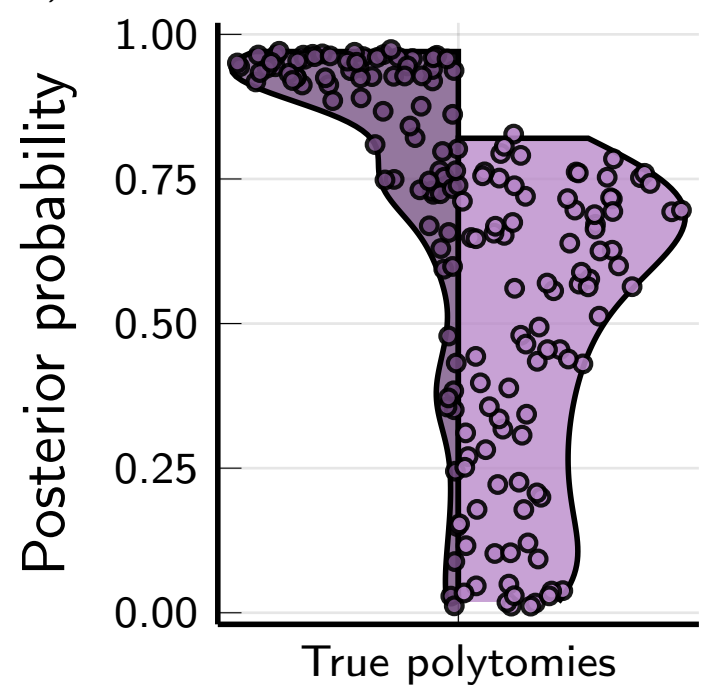

Figure S9. The performance of the $M_{G}$ and $M_{I B}$ tree models when applied to 100 data sets with 500 loci (each with 100 linked characters) simulated on species trees randomly drawn from the $M_{G}$ tree distribution. For each simulation, the mutation-scaled effective population size $\left(N_{e} \mu\right)$ was drawn from a gamma distribution ( shape $=20$, mean $=0.001$ ) and shared across all the branches of the tree; this distribution was used as the prior in analyses. Plots created using the PGFPlotsX (Version 1.2.10, Commit 1adde3d0; Carlsson and Papp, 2021) backend of the Plots (Version 1.5.7, Commit f80ce6a2; Breloff, 2021) package in Julia (Version 1.5.4; Bezanson et al., 2017). 


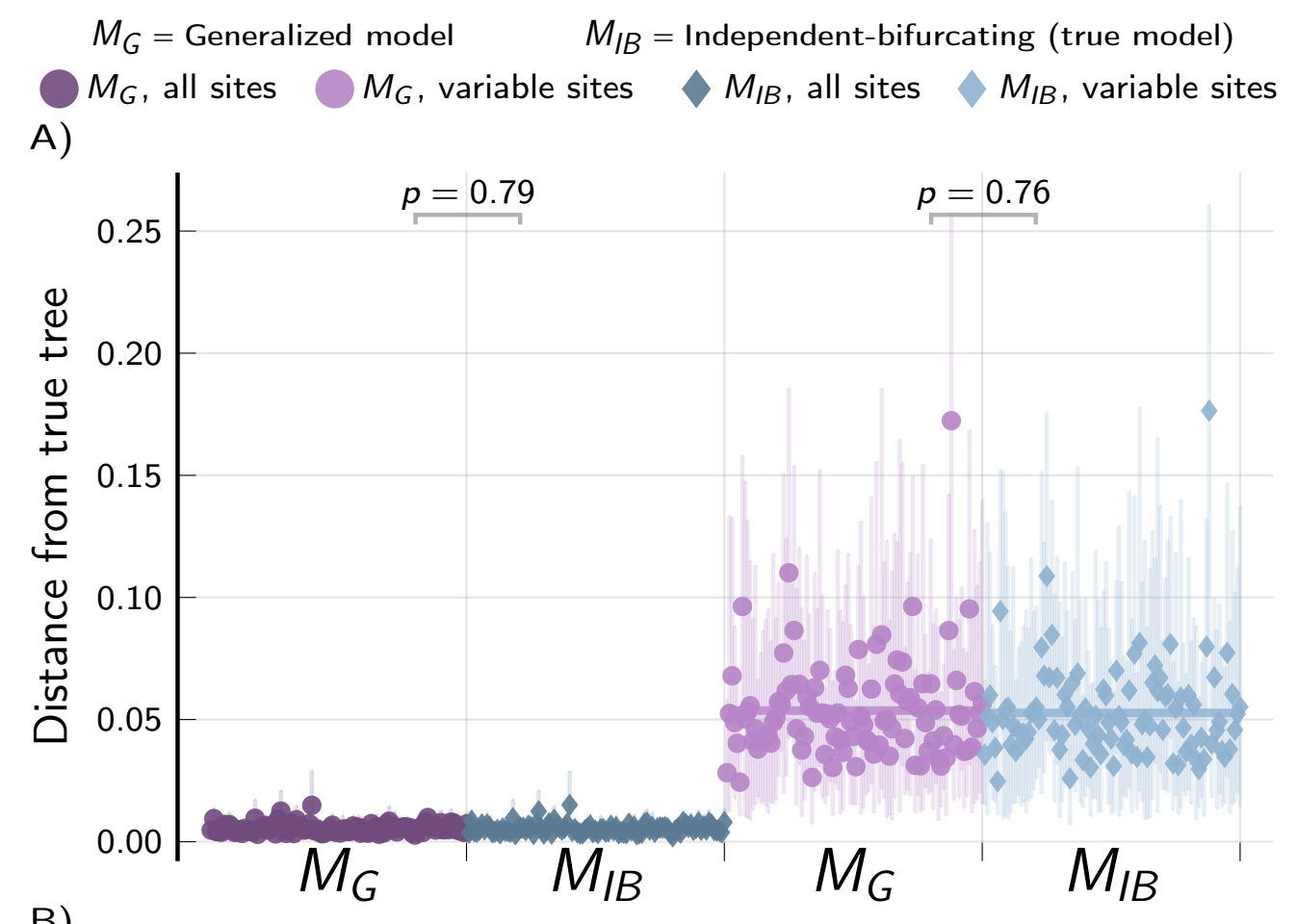

B)

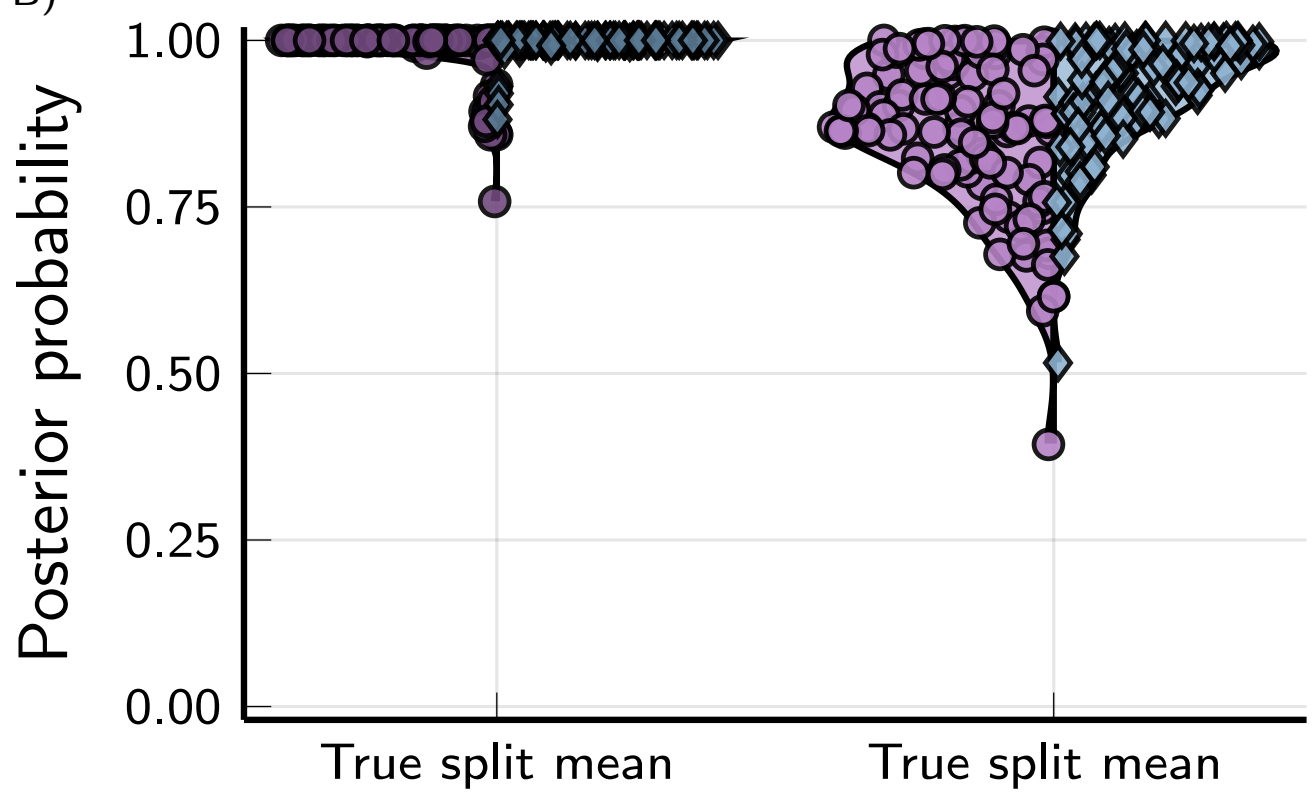

Figure S10. The performance of the $M_{G}$ and $M_{I B}$ tree models when applied to 100 data sets with 500 loci (each with 100 linked characters) simulated on species trees randomly drawn from the $M_{I B}$ tree distribution. For each simulation, the mutation-scaled effective population size $\left(N_{e} \mu\right)$ was drawn from a gamma distribution ( shape $=20$, mean $=0.001$ ) and shared across all the branches of the tree; this distribution was used as the prior in analyses. Plots created using the PGFPlotsX (Version 1.2.10, Commit 1adde3d0; Carlsson and Papp, 2021) backend of the Plots (Version 1.5.7, Commit f80ce6a2; Breloff, 2021) package in Julia (Version 1.5.4; Bezanson et al., 2017). 


\section{Tables}

Table S1. The data for all Cyrtodactylus samples included in our phylogenetic analyses are included in a tab-delimited text file available from the project repository and archived on Zenodo (Oaks and Wood, Jr., 2021): https://raw.githubusercontent.com/phyletica/ gekgo/master/phycoeval-msg-assemblies/ipyrad-assemblies/sample-data/Cyrt_ localities.tsv.

Table S2. The data for all Gekko samples included in our phylogenetic analyses are included in a tab-delimited text file available from the project repository and archived on Zenodo (Oaks and Wood, Jr., 2021): https://raw.githubusercontent.com/phyletica/gekgo/master/ phycoeval-msg-assemblies/ipyrad-assemblies/sample-data/Gekko_localities.tsv. 
Table S3. Settings used for assembling RADseq loci for Cyrtodactylus and Gekko.

\begin{tabular}{ll} 
ipyrad setting & Value \\
\hline assembly_method & denovo \\
datatype & rad \\
restriction_overhang & TATG, \\
max_low_qual_bases & 5 \\
phred_Qscore_offset & 33 \\
mindepth_statistical & 6 \\
mindepth_majrule & 6 \\
maxdepth & 10000 \\
clust_threshold & 0.85 \\
max_barcode_mismatch & 0 \\
filter_adapters & 1 \\
filter_min_trim_len & 35 \\
max_alleles_consens & 2 \\
max_Ns_consens & 0.05 \\
max_Hs_consens & 0.05 \\
min_samples_locus & 4 \\
max_SNPs_locus & 0.2 \\
max_Indels_locus & 8 \\
max_shared_Hs_locus & 0.5 \\
trim_reads & $0,0,0,0$ \\
trim_loci & $0,0,0,0$ \\
\hline
\end{tabular}

Table S4. Convergence statistics we calculated with sumphycoeval from MCMC samples collected with phycoeval. We ran each MCMC chain for 15,000 generations, sampling every 10 generations.

\begin{tabular}{lll} 
Summary & Cyrtodactylus & Gekko \\
\hline Number of chains & 25 & 25 \\
Samples skipped per chain & 101 & 101 \\
Samples retained per chain & 1400 & 1400 \\
Total samples & 35000 & 35000 \\
ASDSF & 0.0027 & 0.00093 \\
Root age PSRF & 1.0000057 & 1.0001458 \\
Root age ESS & 33671.16 & 26844.22 \\
Population size PSRF & 1.0006948 & 1.0006295 \\
Population size ESS & 17542.23 & 13789.14 \\
\hline
\end{tabular}




\section{The generalized tree model}

Let $T$ represent a rooted, potentially multifurcating tree topology with $N$ tips and $n(t)$ internal nodes $\boldsymbol{t}=t_{1}, t_{2}, \ldots t_{n(t)}$, where $n(t)$ can range from 1 (the "comb" tree) to $N-1$ (fully bifurcating, independent divergences). Each internal node $t$ is assigned to one divergence time $\tau$, which it may share with other internal nodes in the tree. We will use $\boldsymbol{\tau}=\tau_{1}, \ldots, \tau_{n(\tau)}$ to represent $n(\tau)$ divergence times, where $n(\tau)$ can also range from 1 to $N-1$, and every $\tau$ has at least one node assigned to it, and every node maps to a divergence time more recent than its parent (Figure S11). For convenience, we will index each $\tau$ from youngest to oldest. We assume the tree is ultrametric; all tips are at time zero, which we will denote as $\tau_{0}$.

We assume the age of the root node follows a parametric distribution (e.g., a gamma distribution), and each of the other divergence times is beta-distributed between the present $\left(\tau_{0}\right)$ and the height of the youngest parent of a node mapped to the divergence time (Figure S11). For additional flexibility, we allow a distribution to be placed on the alpha parameter of the beta distributions of all the non-root divergence times, which we denote as $\alpha_{\tau}$. We assume all possible topologies $(T)$ are equally probable.

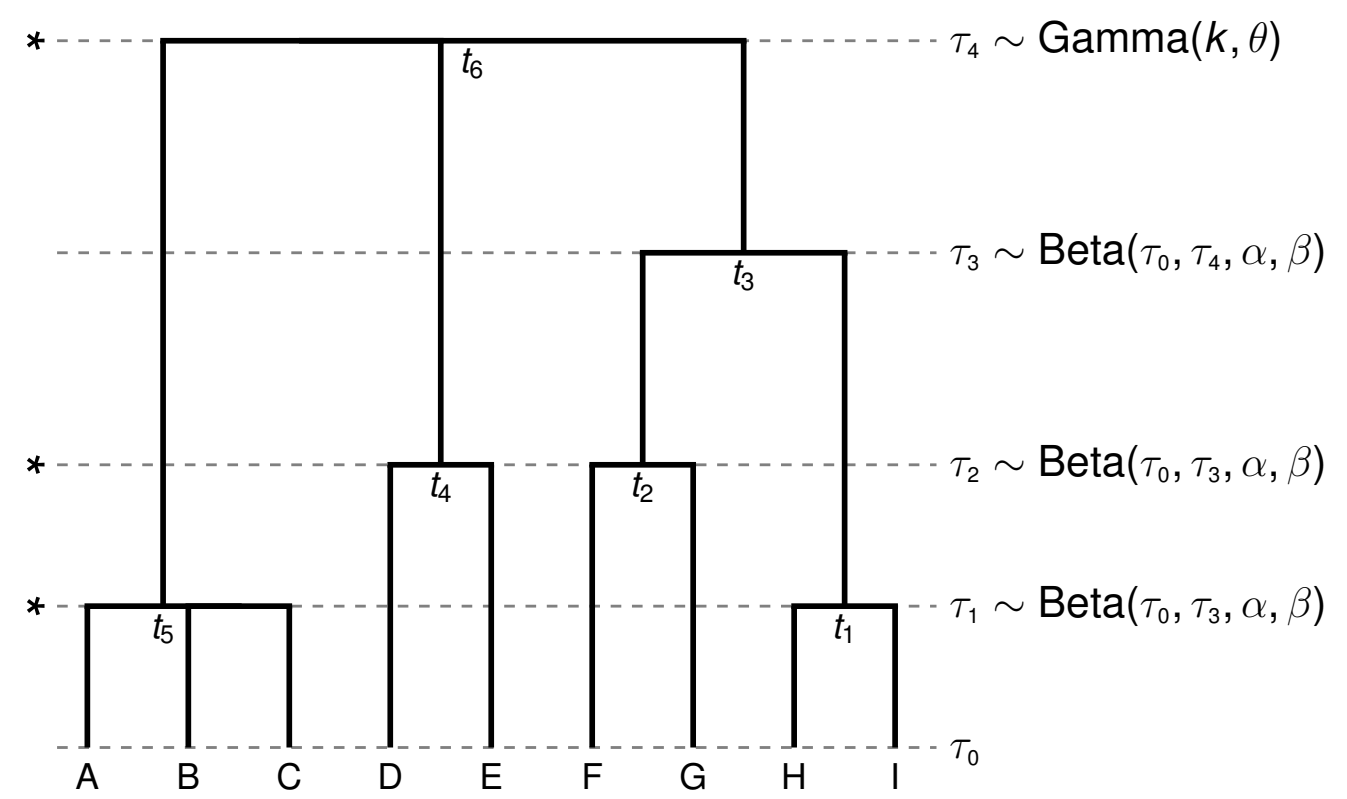

Figure S11. An illustration of the generalized tree model implemented in ecoevolity. The prior distributions of the divergence times are shown to the right, and "splittable" divergence times are indicated with an asterisk to the left. Figure created using Gram (Version 4.0.0, Commit 02286362; Foster, 2018) and the P4 phylogenetic toolkit (Version 1.4, Commit d9c8d1b1; Foster, 2004). 


\section{Approximating the posterior of the generalized tree model}

Below we describe Markov chain Monte Carlo algorithms for sampling from the generalized tree distribution, including reversible-jump moves for moving between trees with different numbers of divergence times. For each we derive the prior and Hastings ratio. These moves can be coupled with any likelihood function to calculate the likelihood ratio and sample from the posterior distribution of generalized trees.

\subsection{Split-time move}

To generalize the space of trees, we introduce two reversible-jump moves: "split-time" and "merge-times." When a reversible-jump move is to be attempted, the merge-times or split-time move is chosen with probability 0.5 , except for two special cases:

1. If the current state of the chain is the most general tree model $(n(\tau)=N-1)$, then the merge-times move is chosen with probability 1 .

2. If the current state of the chain is the "comb" tree $(n(\tau)=1)$, then the split-time move is chosen with probability 1 .

The basic idea is to randomly divide a "splittable" divergence time into two nonempty sets of nodes, and assign one of the sets to a new, more recent divergence time. A divergence time is considered splittable if it has (1) more than one node mapped to it, or (2) a single multifurcating node mapped to it (Figure S11). The first step is to randomly choose a divergence time, $\tau_{i}$, from among the splittable divergence times. After dividing $\tau_{i}$ into two sets of nodes, we need to randomly select a time more recent than $\tau_{i}$ to assign one of the sets.

\subsubsection{Drawing the new divergence time}

To get the new, proposed divergence time, we randomly draw a new divergence time between $\tau_{i-1}$ and $\tau_{i}$ from a proposal distribution, where

$$
g_{\tau}\left(\tau^{\prime} \mid \tau_{i}, \tau_{i-1}\right)
$$

is the conditional probability density of proposing the new time $\tau^{\prime}$ given the times from the current values of $\tau_{i}$ and $\tau_{i-1}$. In our implementation, we use a beta probability distribution scaled and shifted to the interval $\tau_{i-1}-\tau_{i}$, so that the probability density of the new time is

$$
g_{\tau}\left(\tau^{\prime} \mid \tau_{i}, \tau_{i-1}, \alpha, \beta\right)=\frac{\left(\frac{\tau^{\prime}-\tau_{i-1}}{\tau_{i}-\tau_{i-1}}\right)^{\alpha-1}\left(1-\frac{\tau^{\prime}-\tau_{i-1}}{\tau_{i}-\tau_{i-1}}\right)^{\beta-1}}{B(\alpha, \beta)\left(\tau_{i}-\tau_{i-1}\right)},
$$

where $\alpha$ and $\beta$ are the two positive shape parameters of the beta distribution, and $B(\alpha, \beta)$ is the beta function that serves as a normalizing constant. For generality and simplicity, we will use $g_{\tau}$ to denote this probability density of the proposed divergence time below. 


\subsubsection{Prior ratio}

The prior ratio for the split-time move is

$$
\frac{f\left(T^{\prime}, \boldsymbol{\tau}^{\prime}\right)}{f(T, \boldsymbol{\tau})},
$$

where $f\left(T^{\prime}, \boldsymbol{\tau}^{\prime}\right)$ is the prior probability of the proposed tree topology and divergence times. In our implementation, we assume that all possible tree topologies (across $n(\tau)=1,2, \ldots, N-1$ ) are equally probable a priori. We also assume the divergence time of the root $\left(\tau_{n(\tau)}\right)$ is gamma-distributed, and each of the other divergence times is beta-distributed between the present $\left(\tau_{0}\right)$ and the height of the youngest parent of a node mapped to the divergence time (Figure S11). Given these assumptions, and using $y\left(\tau_{i}\right)$ to represent the divergence time of the youngest parent of the nodes mapped to $\tau_{i}$, the prior ratio becomes

$$
\frac{f\left(\tau^{\prime} \mid y\left(\tau^{\prime}\right)\right) f\left(\tau_{i-1} \mid y\left(\tau_{i-1}\right)^{\prime}\right)}{f\left(\tau_{i-1} \mid y\left(\tau_{i-1}\right)\right)},
$$

If $\tau_{i}=1$ (i.e., the divergence time selected to split was the most recent divergence), then $\tau_{i-1}$ is the present, and so $f\left(\tau_{i-1} \mid y\left(\tau_{i-1}\right)^{\prime}\right)=f\left(\tau_{i-1} \mid y\left(\tau_{i-1}\right)\right)=1$. Also, if none of the nodes assigned to $\tau_{i-1}$ has a parent assigned to the newly proposed divergence time (i.e., $\left.y\left(\tau_{i-1}\right)^{\prime} \neq \tau^{\prime}\right)$, then $y\left(\tau_{i-1}\right)^{\prime}=y\left(\tau_{i-1}\right)$; e.g., in Figure $\mathrm{S} 11$, if $\tau_{2}$ is split, the prior probability density of $\tau_{1}$ is not affected, because the youngest parent of a node mapped to $\tau_{1}$ is mapped to $\tau_{3}$. In both of these special cases, the prior ratio further simplifies to

$$
f\left(\tau^{\prime} \mid y\left(\tau^{\prime}\right)\right)
$$

\subsubsection{Hastings ratio}

The probability of proposing a split-time move involves several components. First, we have to choose to split rather than merge. We will account for the probability of this toward the end of this section. Next, we randomly choose a splittable divergence time $\tau_{i}$ with probability $\frac{1}{n_{s}(\tau)}$, where $n_{s}(\tau)$ is the number of splittable divergence times. As described in Section 4.1 .1 above, we randomly choose a new divergence time $\tau^{\prime}$ more recent than $\tau_{i}$ with probability density $g_{\tau}$.

When we divide $\tau_{i}$ into two sets of nodes, if any polytomies get broken up, new branches will get added to the tree. Under certain models, each of these new branches will need values randomly drawn for parameters. For example, if using a "relaxed-clock" model, each new branch will need a substitution rate. Or, if using a multi-species coalescent model where each branch has its own effective population size, a value for this will need to be drawn. Note, this does not involve divergence-time parameters, because all nodes split from $\tau_{i}$ will be assigned to $\tau^{\prime}$. We will use $\boldsymbol{g}_{z}$ to represent the product of all the probability densities of the proposed values for the new branches. If no polytomies get broken up, or new branches created from broken polytomies do not require parameter values, $\boldsymbol{g}_{z}=1$.

We will deal with how the nodes assigned to $\tau_{i}$ are divided into two sets below. For now, we will use $\Xi$ to represent the probability of the proposed division of $\tau_{i}$. The probability 
density of the proposed split move is then

$$
g\left(\Theta^{\prime} \mid \Theta\right)=\frac{\boldsymbol{g}_{z} g_{\tau} \Xi}{n_{s}(\tau)},
$$

where $\Theta$ and $\Theta^{\prime}$ represent the full state of the model before and after the proposed split move, respectively. The move that would exactly reverse this split move would simply entail randomly selecting the proposed divergence time from all divergence times except the root, which would then be deterministically merged with the next older divergence time. The probability of this reverse move is

$$
g\left(\Theta \mid \Theta^{\prime}\right)=\frac{1}{n(\tau)^{\prime}-1}=\frac{1}{n(\tau)}
$$

where $n(\tau)$ and $n(\tau)^{\prime}$ is the number of divergence times before and after the proposed split move, respectively.

The Hastings ratio for the split move is then

$$
\frac{g\left(\Theta \mid \Theta^{\prime}\right)}{g\left(\Theta^{\prime} \mid \Theta\right)}=\gamma_{S} \frac{n_{s}(\tau)}{n(\tau) \boldsymbol{g}_{z} g_{\tau} \Xi},
$$

where $\gamma_{S}$ represents the probability of choosing to merge in the reverse move divided by the probability of choosing to split in the forward move, which is

$\gamma_{S}= \begin{cases}0.5 & \text { if current tree is the "comb" and proposed tree has } n(\tau)<N-1 \\ 2.0 & \text { if proposed tree has } n(\tau)=N-1 \text { and the current tree is not the "comb" } \\ 1.0 & \text { otherwise. }\end{cases}$

\subsection{Merge-times move}

In the "merge-times" move, we randomly choose $\tau_{x}$ from one of the $n(\tau)-1$ non-root divergence times. Then, we merge $\tau_{x}$ with the next older divergence time, $\tau_{x+1}$. This will create shared divergence times among nodes and/or multifurcating nodes. We will use $\tau_{x+1}^{\prime}$ to refer to the newly merged divergence time proposed by the move.

\subsubsection{Prior ratio}

Generally, the prior ratio for the merge-times move is the same as Equation 5. Assuming (1) all topologies are equally probable, $(2)$ the divergence time of the root $\left(\tau_{n(\tau)}\right)$ is gammadistributed, and (3) each of the other divergence times is beta-distributed between the present $\left(\tau_{0}\right)$ and the height of the youngest parent to the nodes mapped to the divergence time (Figure S11), the prior ratio becomes

$$
\frac{f\left(\tau_{x+1}^{\prime} \mid y\left(\tau_{x+1}^{\prime}\right)\right)}{f\left(\tau_{x} \mid y\left(\tau_{x}\right)\right) f\left(\tau_{x+1} \mid y\left(\tau_{x+1}\right)\right)} .
$$


If $\tau_{x+1}$ is the root of the tree, then $f\left(\tau_{x+1}^{\prime} \mid y\left(\tau_{x+1}^{\prime}\right)\right)=f\left(\tau_{x+1}^{\prime}\right)=f\left(\tau_{x+1} \mid y\left(\tau_{x+1}\right)\right)=f\left(\tau_{x+1}\right)$, and this probability density is given by the gamma prior distribution on the divergence time of the root (Figure S11).

\subsubsection{Hastings ratio}

The probability of the forward merge-times move is simply

$$
g\left(\Theta^{\prime} \mid \Theta\right)=\frac{1}{n(\tau)-1}=\frac{1}{n(\tau)^{\prime}}
$$

where $n(\tau)$ and $n(\tau)^{\prime}$ is the number of divergence times before and after the proposed merge move, respectively.

Borrowing from Equation 8, the probability density of the split move that would exactly reverse the proposed merge move is

$$
g\left(\Theta \mid \Theta^{\prime}\right)=\frac{\boldsymbol{g}_{z} g_{\tau} \Xi}{n_{s}(\tau)^{\prime}}
$$

where $n_{s}(\tau)^{\prime}$ is the number of splittable divergence times after the proposed merge-times move.

The Hastings ratio for the merge move is then

$$
\frac{g\left(\Theta \mid \Theta^{\prime}\right)}{g\left(\Theta^{\prime} \mid \Theta\right)}=\gamma_{M} \frac{n(\tau)^{\prime} \boldsymbol{g}_{z} g_{\tau} \Xi}{n_{s}(\tau)^{\prime}}
$$

where $\gamma_{M}$ represents the probability of choosing to split in the reverse move divided by the probability of choosing to merge in the forward move, which is

$$
\gamma_{M}= \begin{cases}2.0 & \text { if proposed tree is the "comb" and current tree has } n(\tau)<N-1 \\ 0.5 & \text { if current tree has } n(\tau)=N-1 \text { and the proposed tree is not the "comb" } \\ 1.0 & \text { otherwise }\end{cases}
$$

\subsection{Expanding $\Xi$}

Up to this point, we have not dealt with how, during the split-time move, we divide the nodes mapped to $\tau_{i}$ into two sets, one of which gets assigned to the new divergence time drawn between $\tau_{i-1}$ and $\tau_{i}$. This has to be done with care to ensure that every possible configuration of two divergence times derived from the nodes assigned to $\tau_{i}$ can be proposed, such that it properly balances the reverse merge-times move. As above, we use $\Xi$ to represent the probability of the proposed division of $\tau_{i}$ 's nodes.

In the next two sections, we show how this is done for two special cases. The first special case illustrates how we first choose which nodes currently mapped to $\tau_{i}$ will get moved to the new divergence time. The second special case shows how we handle any multifurcating 
nodes that have been chosen to be moved to the new divergence time. In the third section, we build on these special cases to show a general solution for $\Xi$.

\subsubsection{The case of all bifurcating nodes mapped to $\tau_{i}$}

We will use $n\left(t \mapsto \tau_{i}\right)$ to represent the number of nodes mapped to $\tau_{i}$. If all $n\left(t \mapsto \tau_{i}\right)$ nodes mapped to $\tau_{i}$ are bifurcating, we randomly divide these nodes into two non-empty sets and then randomly choose one of the two sets of nodes to move to the new divergence time. For example, this would be the case if $\tau_{2}$ is chosen to split from the tree shown in Figure S11.

The number of ways $n\left(t \mapsto \tau_{i}\right)$ can be divided into two non-empty subsets is given by the Stirling number of the second kind, which we denote as $S_{2}\left(n\left(t \mapsto \tau_{i}\right), 2\right)$. We uniformly choose among these, such that the probability of randomly selecting any set partition of the $n\left(t \mapsto \tau_{i}\right)$ nodes mapped to $\tau_{i}$ is $\frac{1}{n\left(t \mapsto \tau_{i}\right)}$. After partitioning the nodes into two sets, there is a $1 / 2$ probability of choosing one set to move to the new, more recent divergence time. Thus, when all of the nodes mapped to $\tau_{i}$ are bifurcating the probability of each possible splitting of $\tau_{i}$ is

$$
\Xi=\frac{1}{2 \times S_{2}\left(n\left(t \mapsto \tau_{i}\right), 2\right)}=\frac{1}{2^{n\left(t \mapsto \tau_{i}\right)}-2}
$$

\subsubsection{The case of a single polytomy mapped to $\tau_{i}$}

Next, let's consider another special case where the number of nodes mapped to $\tau_{i}$ is one (i.e., a single polytomy). For example, this would be the case if $\tau_{4}$ is chosen to split from the tree shown in Figure S11. In this case, we randomly resolve the polytomy, by randomly (uniformly) choosing a set partition of the descending branches into non-empty subsets. Any subsets with only one branch remain attached to the original polytomy node, while each subset with multiple branches get split off to form a new node (clade) that descends from the original polytomy node. These new nodes are assigned to the new, more recent divergence time $\tau^{\prime}$. The number of ways to partition the descending branches of the polytomy are thus $B_{b}-2$, where $B_{b}$ is the Bell number (Bell, 1934) - the number of possible set partitions of the $b$ branches descending from the polytomy. We have to subtract 2 from $B_{b}$, because we do not allow the two "extreme" set partitions with one or $b$ subsets. The former would move the whole polytomy to the new divergence time, and the latter would leave the polytomy as is. Neither of these scenarios adds a dimension (divergence time) to the model. We avoid these two scenarios using rejection so that the remaining partitions of the $b$ descending branches are chosen uniformly. Thus, when only a single polytomy is mapped to $\tau_{i}$ the probability of each possible splitting of $\tau_{i}$ is

$$
\Xi=\frac{1}{B_{b}-2}
$$

\subsubsection{The case when multiple nodes, including at least one polytomy, is mapped to $\tau_{i}$}

When multiple nodes are mapped to $\tau_{i}$, and at least one is a polytomy, we need to do some more accounting to ensure that we can reach every possible arrangement of two divergence 
times that can be merged to form the current configuration of nodes mapped to $\tau_{i}$. Similar to the case with all bifurcating nodes, we will first divide the $n\left(t \mapsto \tau_{i}\right)$ nodes mapped to $\tau_{i}$ into two subsets and randomly choose one of these subsets to move to the proposed, more recent divergence time, $\tau^{\prime}$. For each multifurcating node that ends up in the subset to be moved to $\tau^{\prime}$ (if any), we need to either break up the polytomy, as we did in the case of the single-polytomy case above, or move the entire polytomy to $\tau^{\prime}$.

Unlike in the case of only bifurcating nodes mapped to $\tau_{i}$, when we partition the $n\left(t \mapsto \tau_{i}\right)$ nodes mapped to $\tau_{i}$ into two sets, we must allow for the case where all $n\left(t \mapsto \tau_{i}\right)$ end up in the set to move to $\tau^{\prime}$. This is because, if any of the polytomy nodes get broken up, they will leave at least one node at $\tau_{i}$, and the dimension of the model will change (i.e., the number of divergence times will increase by one). So, we have to allow an empty subset when we randomly partition the $n\left(t \mapsto \tau_{i}\right)$ into two subsets. However, we cannot allow the empty subset to be chosen to move to $\tau^{\prime}$. There are $S_{2}\left(n\left(t \mapsto \tau_{i}\right), 2\right)+1$ ways to partition the $n\left(t \mapsto \tau_{i}\right)$ nodes mapped to $\tau_{i}$ into two subsets if we allow the set partition with one empty subset. For each of these, there are two ways to choose the subset to move to $\tau^{\prime}$, and of all of these, there is one scenario we will reject: if the empty set gets selected to move to $\tau^{\prime}$. Thus, there are

$$
\left(2\left(S_{2}\left(n\left(t \mapsto \tau_{i}\right), 2\right)+1\right)\right)-1=\left(2 S_{2}\left(n\left(t \mapsto \tau_{i}\right), 2\right)\right)+1=2^{n\left(t \mapsto \tau_{i}\right)}-1
$$

ways to choose a subset of the nodes assigned to $\tau_{i}$ for moving to the new divergence time, and the probability of each is

$$
\frac{1}{2^{n\left(t \mapsto \tau_{i}\right)}-1} .
$$

For each polytomy mapped to $\tau_{i}$ that ends up in the set of nodes to move to $\tau^{\prime}$ (if any), we randomly choose one of the $B_{b}$ possible set partitions of the $b$ branches descending from the polytomy. However, we will reject the set partition with $b$ subsets (i.e., all branches end up in their own subset). We reject this, because no subclades get broken off from the polytomy to move to $\tau^{\prime}$, and this scenario is already taken into account by the polytomy node not ending up in the set of nodes to move to $\tau^{\prime}$ in the first place. However, we need to allow the scenario where all $b$ branches descending from a polytomy get assigned to a single set, which results in the entire polytomy node getting moved to $\tau^{\prime}$, as long as at least one node remains assigned to $\tau_{i}$ (we will handle this in a bit). Thus, for each polytomy in the set of nodes to be moved to $\tau^{\prime}$, there are $B_{b}-1$ ways to move it. Using $n_{p}\left(t \Rightarrow \tau^{\prime}\right)$ to represent the number of polytomies in the subset of nodes to be moved to $\tau^{\prime}$, the total number of ways these polytomies can be moved to $\tau^{\prime}$ is

$$
\Phi=\prod_{x=1}^{n_{p}\left(t \Rightarrow \tau^{\prime}\right)}\left(B_{b_{x}}-1\right)
$$


and the probability of each is equal to

$$
\prod_{x=1}^{n_{p}\left(t \Rightarrow \tau^{\prime}\right)} \frac{1}{\left(B_{b_{x}}-1\right)}=\frac{1}{\prod_{x=1}^{n_{p}\left(t \Rightarrow \tau^{\prime}\right)}\left(B_{b_{x}}-1\right)}=\frac{1}{\Phi}
$$

If no polytomies end up in the subset of nodes to move to $\tau^{\prime}$, then $\Phi=1$.

However, if all $n\left(t \mapsto \tau_{i}\right)$ nodes mapped to $\tau_{i}$ end up in the set of nodes to be moved to $\tau^{\prime}$, we need to reject the case where none of the polytomy nodes gets broken up (i.e., for every polytomy, all the descending branches get partitioned into a single set), because no nodes would remain assigned to $\tau_{i}$, and the move would simplify to changing the value of $\tau_{i}$. Thus, if all $n\left(t \mapsto \tau_{i}\right)$ nodes mapped to $\tau_{i}$ end up in the set of nodes to move to $\tau^{\prime}$, the total number of ways all $n_{p}\left(t \Rightarrow \tau^{\prime}\right)$ polytomies can be moved to $\tau^{\prime}$ is

$$
\left(\prod_{x=1}^{n_{p}\left(t \Rightarrow \tau^{\prime}\right)}\left(B_{b_{x}}-1\right)\right)-1=\Phi-1
$$

and the probability of each is equal to

$$
\frac{1}{\Phi-1}
$$

Given all of this, the probability of choosing a subset of nodes from $\tau_{i}$ to move to the new divergence time across all possible cases is

$\Xi= \begin{cases}\frac{1}{2^{n\left(t \mapsto \tau_{i}\right)}-2} & \text { if no polytomies mapped to } \tau_{i} \\ \frac{1}{\left(2^{n\left(t \mapsto \tau_{i}\right)}-1\right)(\Phi-1)} & \text { if } \geq 1 \text { polytomy nodes mapped to } \tau_{i}, \text { and all } n\left(t \mapsto \tau_{i}\right) \text { assigned to move set } \\ \frac{1}{\left(2^{n\left(t \mapsto \tau_{i}\right)}-1\right) \Phi} & \text { otherwise. }\end{cases}$

Notice, the case of $n\left(t \mapsto \tau_{i}\right)=1$ (i.e., only a single polytomy node mapped to $\tau_{i}$ ) is simply a special case of the second condition above, where all the nodes assigned to $\tau_{i}$ end up in the set to move to the new divergence time, including at least one polytomy.

\subsection{Validation of Split-time and Merge-times moves}

To validate the split-time/merge-times moves, we used them to sample from the prior distribution of trees with 5, 6, and 7 leaves. If working correctly, we should sample all $n(T)$ tree topologies with an equal frequency of $\frac{1}{n(T)}$. If we collect $\mathcal{N}$ MCMC samples from the prior distribution, the number of times a topology is sampled should be approximately distributed as $\operatorname{Binomial}\left(n=\mathcal{N}, p=\frac{1}{n(T)}\right)$; i.e., binomially distributed where the number of "trials" is equal to the number of samples, and the probability of sampling each topology is $\frac{1}{n(T)}$. We found a close match between the number or times each tree was sampled by our reversible-jump MCMC chain and the expected number, and failed to reject the expected 
binomial distribution using $\chi^{2}$ goodness-of-fit test (Figure S12; $p=0.742,0.464$, and 0.172 for the test with a 5, 6, and 7-leaved tree).
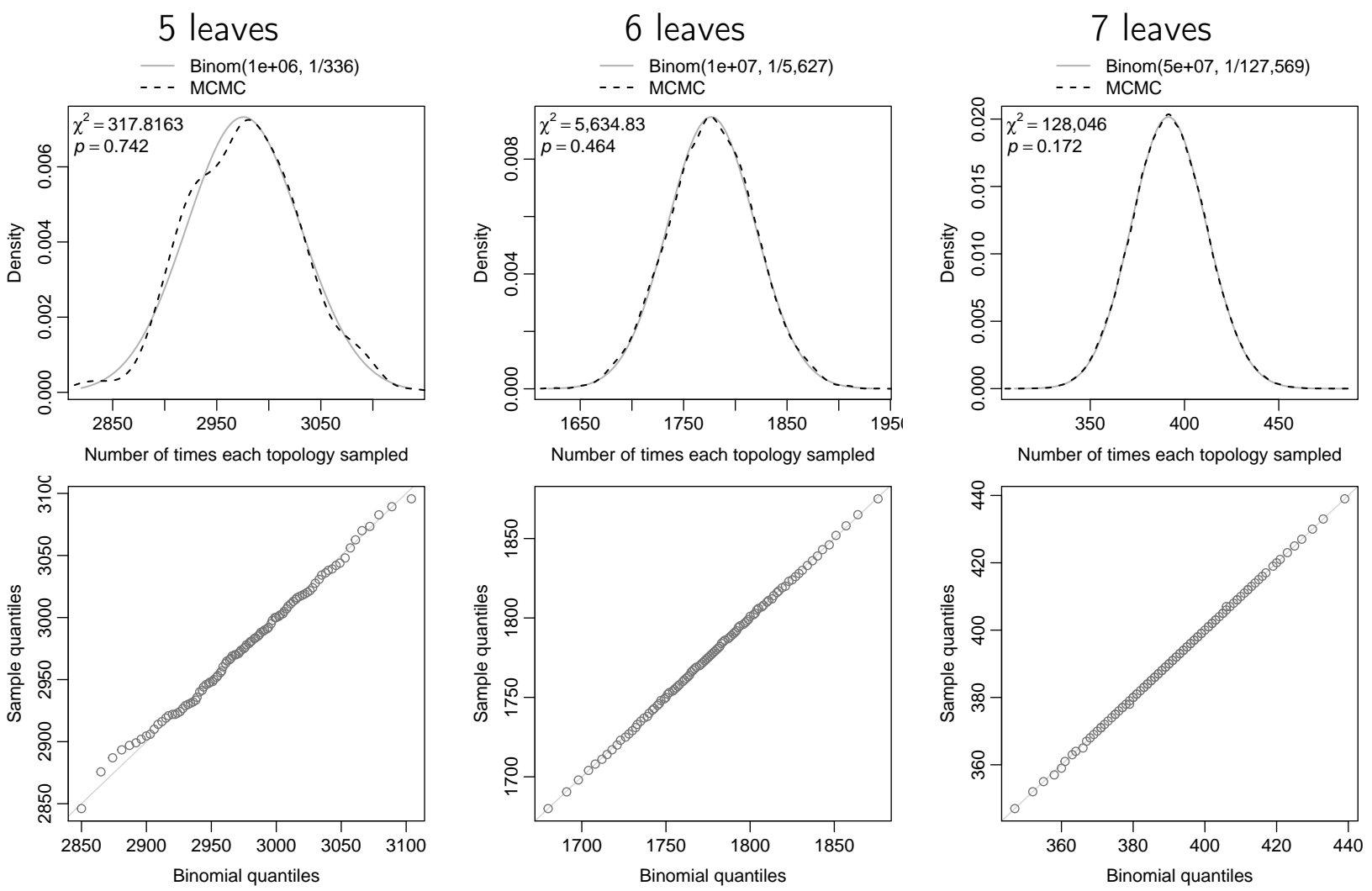

Figure S12. Comparing the expected to the observed number of times each topology is sampled by an MCMC chain using our split-time and merge-times moves. Under our generalized tree distribution, how often each topology is sampled should follow a $\operatorname{Binomial}\left(n=\mathcal{N}, p=\frac{1}{n(T)}\right)$ distribution, where $\mathcal{N}$ is the total number of MCMC samples.

\subsection{Nested-neighbor-node-swap move}

The split-time and merge-times moves can sample all of the space of the generalized tree distribution (assuming the age of the root node is fixed). However, we implemented additional topology moves that do not jump between tree models with different numbers of divergence times.

The goal of this move is to change the topology without changing the number or timing of divergences. We start by randomly picking a non-root divergence time, $\tau_{i}$, with probability $\frac{1}{n(\tau)-1}$. Next, we find the divergence time, $\tau_{j}$, that contains the node that is the youngest parent of nodes mapped to $\tau_{i}$. We then randomly pick one of the nodes mapped to $\tau_{j}$ that has children mapped to $\tau_{i}$, we will call it $t_{a}$. Each child of $t_{a}$ that is mapped to $\tau_{i}$ will randomly contribute one of its children to a "swap pool" of nodes. If $t_{a}$ has children that are not mapped to $\tau_{i}$, we randomly pick one of these children and add it to the swap pool if it is younger than $\tau_{i}$. If the selected child of $t_{a}$ is older than $\tau_{i}$, we randomly sample one of its 
children and continue to do so until we have chosen a descendant node that is younger than $\tau_{i}$, which we then add to the swap pool. Lastly, we randomly pick two nodes from the swap pool and we swap their parents.

After the proposed move, the structure of the tree rootward of the swapped nodes is the same. Because of this, the move that would reverse the proposed move would be equally probable; it would involve (1) choosing the same non-root divergence time $\tau_{i}$, (2) choosing the same $t_{a}$, (3) choosing the children that swapped parents in the forward move to enter the swap pool, and (4) picking the nodes that swapped parents in the forward move from the pool and swapping their parents back. In $\# 3$, all the parent nodes involved have the same number of children as before the proposed forward move, and so the probability of the reverse move will be equal. As a result, the Hastings ratio for the move is 1 .

For example, for the tree in Figure S11 if we randomly selected $\tau_{1}$, the divergence containing the youngest parent of the nodes mapped to $\tau_{1}$ is $\tau_{3}$. Divergence time $\tau_{3}$ only has one node that is a parent of nodes assigned to $\tau_{1}$, which is $t_{3}$. Node $t_{3}$ only has one child mapped to $\tau_{1}\left(t_{1}\right)$, which will randomly contribute one of its children, Leaf $\mathrm{H}$ or $\mathrm{I}$, to the swap pool. Node $t_{3}$ also has children that are not mapped to $\tau_{1}$, one child $t_{2}$ that is mapped to $\tau_{2}$. Node $t_{2}$ is considered for the swap pool, but it is too old (it is older than $\tau_{1}$ and thus could not become a child of $t_{1}$. So, we randomly consider one the children of $t_{2}$, Leaf F or G, for the swap pool, either of which is young enough to be added to the swap pool. Next, we randomly choose two nodes from the swap pool, which has exactly two nodes in this case, a child of $t_{1}$ (Leaf $\mathrm{H}$ or I) and $t_{2}$ (Leaf $\mathrm{F}$ or $\mathrm{G}$ ), and these nodes swap parents. If we assume that Leaves $\mathrm{G}$ and $\mathrm{H}$ were swapped, it is clear that the probability of the reverse move that would swap them back is equally probable.

We also implemented variations of this move that make larger changes to the tree topology. For example, we can perform the swap for all of the nodes mapped to $\tau_{j}$ that have children mapped to $\tau_{i}$ (instead of randomly choosing one of them). Another option is to randomly permute the parents of all of the nodes in the swap pool, rather than swap the parents of just two of the nodes. By chance, when doing this permutation of the nodes in the swap pool, it is possible possible to end up with the same topology we started with. To avoid proposing the same state, we iteratively permute the parents of the nodes in the swap pool until we have a new topology (the parents of at least some of the nodes in the swap pool have changed). As for the swap move, this permutation move can be performed on one randomly selected node of $\tau_{j}$ that has children mapped to $\tau_{i}$, or to all of them.

\subsubsection{Validation of move}

To validate this move, we used it to sample from a uniform distribution over the topologies of a 6-leaved bifurcating tree. There are 945 topologies for a rooted, bifurcating tree (Felsenstein, 1978). If the move is working correctly, the number of times we sample each of them should be follow a $\operatorname{Binomial}\left(n=\mathcal{N}, p=\frac{1}{945}\right)$ distribution, where $\mathcal{N}$ is the total number of MCMC samples. From an MCMC sample of 100,000 trees, we found a close match to this expected distribution, and were unable to reject it using a $\chi^{2}$ goodness-of-fit test (Figure S13; $p=0.51$ ). 


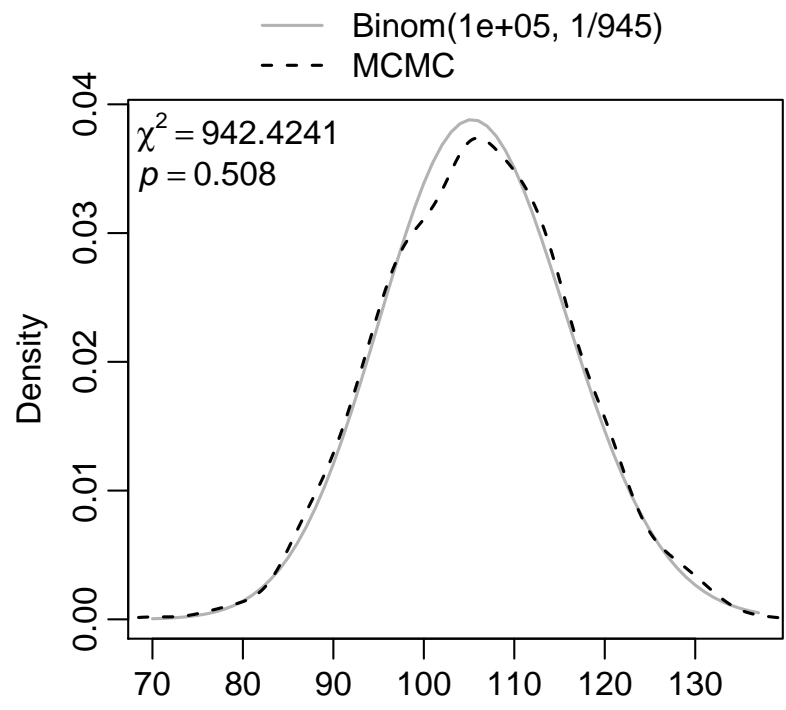

Number of times each topology sampled

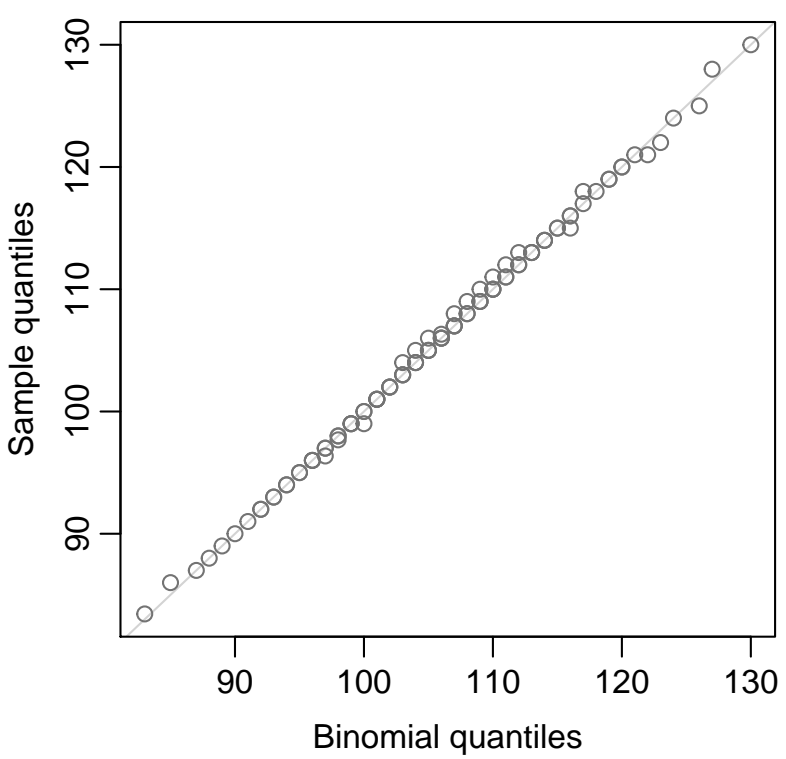

Figure S13. Comparing the expected to the observed number of times each topology of a rooted, 6-leaved, bifurcating tre is sampled by our nested-neighbor-node-swap move. Our MCMC sample of 100,000 trees closely matched the expected $\operatorname{Binomial}\left(n=100,000, p=\frac{1}{945}\right)$ distribution.

\subsection{Divergence time slide bump move}

To begin this move, we randomly pick one of the divergence times, $\tau_{i}$. Next, we draw a uniform deviate, $u \sim$ Uniform $(-\lambda, \lambda)$, where $\lambda$ is a tuning parameter that can be adjusted to improve the acceptance rate of the proposal. Then, we get a new divergence time value by $\tau_{i} e^{u}$. We will index our randomly selected divergence time, $\tau_{i}$, as $\tau_{1}$. We then use $\tau_{1}, \tau_{2}, \ldots, \tau_{n}$ to represent the selected time, $\tau_{1}$, and all the divergence times between $\tau_{1}$ and $\tau_{1} e^{u}$ that contain nodes ancestral or descendant to the nodes mapped to $\tau_{1}$. Note, that incrementing indices count younger or older divergence times, depending on whether $\tau_{i} e^{u}<\tau_{1}$ or $\tau_{i} e^{u}>\tau_{1}$, respectively.

The simplest case is that we do not have any intervening divergence times, and so we only have $\tau_{1}$. This will happen when $\tau_{1} e^{u}$ is older than the oldest node that is a child of the nodes mapped to $\tau_{i}$ and younger than the youngest node that is parent of the nodes mapped to $\tau_{i}$ In that case, we propose a new time to which to slide $\tau_{1}$ as

$$
\tau_{1}^{\prime}=\tau_{1} e^{u}
$$

To reverse this move (slide $\tau_{1}$ back) would be

$$
\tau_{1}=\tau_{1}^{\prime} e^{u^{\prime}},
$$

To solve for the uniform deviate that would exactly reverse the move $\left(u^{\prime}\right)$, we take the $\log$ 
of Equation 27 and solve for $u^{\prime}$.

$$
\begin{aligned}
\ln \left(\tau_{1}\right) & =\ln \left(\tau_{1}^{\prime}\right)+\ln \left(e^{u^{\prime}}\right) \\
\ln \left(\tau_{1}\right) & =\ln \left(\tau_{1}^{\prime}\right)+u^{\prime} \\
u^{\prime} & =\ln \left(\tau_{1}\right)-\ln \left(\tau_{1}^{\prime}\right) \\
u^{\prime} & =\ln \left(\tau_{1}\right)-\ln \left(\tau_{1} e^{u}\right) \\
u^{\prime} & =\ln \left(\tau_{1}\right)-\ln \left(\tau_{1}\right)-u \\
u^{\prime} & =-u .
\end{aligned}
$$

To get the Hastings ratio for this move, we use the formula of Green (1995),

$$
\text { Hastings ratio }=\frac{g^{\prime}\left(u^{\prime}\right)}{g(u)}|\operatorname{det}(J)|,
$$

which is the ratio of the probability of drawing the random deviate that would reverse the proposed move to the probability of drawing the random deviate of the proposed move, multiplied by the absolute value of the determinant of a Jacobian matrix. Because the forward and reverse random deviates are uniform, $\frac{g^{\prime}\left(u^{\prime}\right)}{g(u)}=1$, and the Hastings ratio reduces to just the Jacobian term,

$$
\begin{aligned}
J & =\left[\begin{array}{ll}
\frac{\partial \tau_{1}^{\prime}}{\partial \tau_{1}} & \frac{\partial \tau_{1}^{\prime}}{\partial u} \\
\frac{\partial u^{\prime}}{\partial \tau_{1}} & \frac{\partial u^{\prime}}{\partial u}
\end{array}\right] \\
& =\left[\begin{array}{cc}
e^{u} & \tau_{1} e^{u} \\
0 & -1
\end{array}\right] \\
\operatorname{det}(J) & =-e^{u} \\
|\operatorname{det}(J)| & =\left|-e^{u}\right|=e^{u}=\text { Hastings ratio. }
\end{aligned}
$$

In the next simplest case, there is one intervening divergence time $\tau_{2}$. In this case, $\tau_{1}$ will slide to $\tau_{2}$ and "bump" it to the new time $\tau_{1} e^{u}$. More formally, the move will be:

$$
\begin{aligned}
& \tau_{2}^{\prime}=\tau_{1} e^{u} \\
& \tau_{1}^{\prime}=\tau_{2}
\end{aligned}
$$

Again, the uniform deviate that would exactly reverse this move would be

$$
u^{\prime}=-u
$$

and the reverse move would be

$$
\begin{aligned}
& \tau_{2}=\tau_{1}^{\prime} \\
& \tau_{1}=\tau_{2}^{\prime} e^{u^{\prime}}
\end{aligned}
$$


Again, the Hastings ratio reduces to just the Jacobian term,

$$
\begin{aligned}
J & =\left[\begin{array}{lll}
\frac{\partial \tau_{2}^{\prime}}{\partial \tau_{2}} & \frac{\partial \tau_{2}^{\prime}}{\partial \tau_{1}} & \frac{\partial \tau_{2}^{\prime}}{\partial u} \\
\frac{\partial \tau_{1}^{\prime}}{\partial \tau_{2}} & \frac{\partial \tau_{1}^{\prime}}{\partial \tau_{1}} & \frac{\partial \tau_{1}^{\prime}}{\partial u} \\
\frac{\partial u^{\prime}}{\partial \tau_{2}} & \frac{\partial u^{\prime}}{\partial \tau_{1}} & \frac{\partial u^{\prime}}{\partial u}
\end{array}\right] \\
& =\left[\begin{array}{ccc}
0 & e^{u} & \tau_{1} e^{u} \\
1 & 0 & 0 \\
0 & 0 & -1
\end{array}\right] \\
\operatorname{det}(J) & =0\left[\begin{array}{cc}
0 & 0 \\
0 & -1
\end{array}\right]-e^{u}\left[\begin{array}{cc}
1 & 0 \\
0 & -1
\end{array}\right]+\tau_{1} e^{u}\left[\begin{array}{ll}
1 & 0 \\
0 & 0
\end{array}\right] \\
\operatorname{det}(J) & =e^{u} \\
|\operatorname{det}(J)| & =e^{u}=\text { Hastings ratio. }
\end{aligned}
$$

To generalize this to an arbitrary number of intervening divergence times that will be bumped, we have

$$
\begin{aligned}
& \tau_{n}^{\prime}=\tau_{1} e^{u} \\
& \tau_{n-1}^{\prime}=\tau_{n} \\
& \tau_{2}^{\prime}=\tau_{3} \\
& \tau_{1}^{\prime}=\tau_{2} .
\end{aligned}
$$

Again, the uniform deviate that would exactly reverse this move would be

$$
u^{\prime}=-u
$$

and the reverse move would be

$$
\begin{aligned}
& \tau_{n}=\tau_{n-1}^{\prime} \\
& \tau_{n-1}=\tau_{n-2}^{\prime} \\
& \tau_{2}=\tau_{1}^{\prime} \\
& \tau_{1}=\tau_{n}^{\prime} e^{u^{\prime}} .
\end{aligned}
$$


The Hastings ratio reduces to just the Jacobian term,

$$
\begin{aligned}
& J=\left[\begin{array}{cclccc}
\frac{\partial \tau_{n}^{\prime}}{\partial \tau_{n}} & \frac{\partial \tau_{n}^{\prime}}{\partial \tau_{n-1}} & \cdots & \frac{\partial \tau_{n}^{\prime}}{\partial \tau_{2}} & \frac{\partial \tau_{n}^{\prime}}{\partial \tau_{1}} & \frac{\partial \tau_{n}^{\prime}}{\partial u} \\
\frac{\partial \tau_{n-1}^{\prime}}{\partial \tau_{n}} & \frac{\partial \tau_{n-1}^{\prime}}{\partial \tau_{n-1}} & \cdots & \frac{\partial \tau_{n-1}^{\prime}}{\partial \tau_{2}} & \frac{\partial \tau_{n-1}^{\prime}}{\partial \tau_{1}} & \frac{\partial \tau_{n-1}^{\prime}}{\partial u} \\
\vdots & \vdots & \ddots & \vdots & \vdots & \vdots \\
\frac{\partial \tau_{2}^{\prime}}{\partial \tau_{n}} & \frac{\partial \tau_{2}^{\prime}}{\partial \tau_{n-1}} & \cdots & \frac{\partial \tau_{2}^{\prime}}{\partial \tau_{2}} & \frac{\partial \tau_{2}^{\prime}}{\partial \tau_{1}} & \frac{\partial \tau_{2}^{\prime}}{\partial u} \\
\frac{\partial \tau_{1}^{\prime}}{\partial \tau_{n}} & \frac{\partial \tau_{1}^{\prime}}{\partial \tau_{n-1}} & \cdots & \frac{\partial \tau_{1}^{\prime}}{\partial \tau_{2}} & \frac{\partial \tau_{1}^{\prime}}{\partial \tau_{1}} & \frac{\partial \tau_{1}^{\prime}}{\partial u} \\
\frac{\partial u^{\prime}}{\partial \tau_{n}} & \frac{\partial u^{\prime}}{\partial \tau_{n-1}} & \cdots & \frac{\partial u^{\prime}}{\partial \tau_{2}} & \frac{\partial u^{\prime}}{\partial \tau_{1}} & \frac{\partial u^{\prime}}{\partial u}
\end{array}\right] \\
& =\left[\begin{array}{cccccc}
0 & 0 & \cdots & 0 & e^{u} & \tau_{1} e^{u} \\
1 & 0 & \ddots & 0 & 0 & 0 \\
\vdots & \ddots & \ddots & \ddots & \vdots & \vdots \\
0 & 0 & \ddots & 0 & 0 & 0 \\
0 & 0 & \cdots & 1 & 0 & 0 \\
0 & 0 & \cdots & 0 & 0 & -1
\end{array}\right] \\
& \operatorname{det}(J)=e^{u}\left[\begin{array}{ccccc}
1 & 0 & \ddots & 0 & 0 \\
\vdots & \ddots & \ddots & \ddots & \vdots \\
0 & 0 & \ddots & 0 & 0 \\
0 & 0 & \cdots & 1 & 0 \\
0 & 0 & \cdots & 0 & -1
\end{array}\right]-\tau_{1} e^{u}\left[\begin{array}{ccccc}
1 & 0 & \ddots & 0 & 0 \\
\vdots & \ddots & \ddots & \ddots & \vdots \\
0 & 0 & \ddots & 0 & 0 \\
0 & 0 & \cdots & 1 & 0 \\
0 & 0 & \cdots & 0 & 0
\end{array}\right] \\
& =-e^{u} \\
& |\operatorname{det}(J)|=\left|-e^{u}\right|=e^{u}=\text { Hastings ratio. }
\end{aligned}
$$

We avoid values of $\tau_{1} e^{u}$ that are less than zero, by rejecting the proposed move. There is no upper limit for the move, because the root of the tree can be moved to an arbitrarily old divergence time. However, in our implementation, the prior on the divergence time of the root is different than the other divergence times, and can be much more informative. In such cases, we might be able to improve mixing and tuning of the move be excluding the root divergence time from the move. We do this by only selecting only non-root divergence times, and rejecting any proposed moves where $\tau_{1} e^{u}$ is older than the root.

\subsubsection{An extension to this move}

We can easily extend this move to also propose new topologies. Whenever we have a "bump" that involves a node and its children, we can propose a node swapping or permuting move described above (see the nested-neighbor-node-swap move and its extensions). Because we are sliding the nodes to take the position of the nodes they bump, the swap or permute 
moves are simplified a bit. We do not have to worry about $\tau_{j}$ contributing a child that is older than its potential new parents, so we never need to randomly choose descendants until we find a node that is younger than $\tau_{i}$. We implemented this move, but jointly proposing changes to continuous divergence time parameters and changing the topology might lead to poor acceptance rates (Yang, 2014), so using separate moves to update divergence times and the topology is likely a better strategy.

\subsubsection{Validation of this move}

We used this move to sample from the prior distribution to ensure that the distribution of sampled divergence times matched the gamma-distributed prior we placed on the root age and the beta priors we placed on all other divergence times. To validate the extension of this move that also incorporates node swapping when nodes "bump," we used it to sample from a uniform distribution over the topologies of a 6-leaved bifurcating tree. If the move is working correctly, the number of times we sample each of the 945 topologies should be follow a $\operatorname{Binomial}\left(n=\mathcal{N}, p=\frac{1}{945}\right)$ distribution, where $\mathcal{N}$ is the total number of MCMC samples. We founds a close match between our samples and this expected distribution, and could not reject it using a $\chi^{2}$ goodness-of-fit test (Figure S14; $p=0.165$, ).
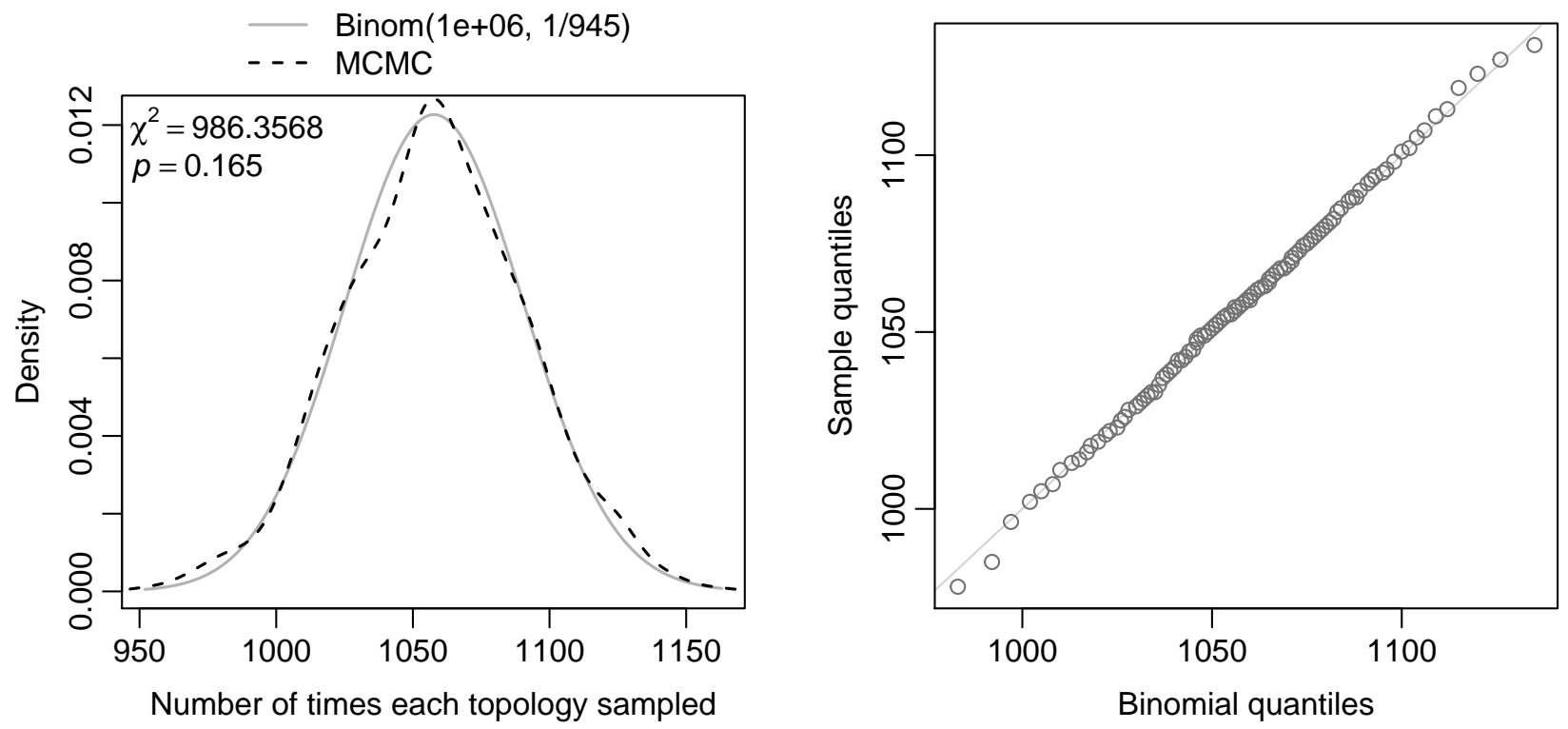

Figure S14. Comparing the expected to the observed number of times each topology of a rooted, 6-leaved, bifurcating tre is sampled by the node-swapping extension to our divergencetime-slide-bump move. Our MCMC sample of 1 million trees closely matched the expected $\operatorname{Binomial}\left(n=1 \times 10^{6}, p=\frac{1}{945}\right)$ distribution. 